\title{
The Maskit embedding of the twice punctured torus
}

\author{
CAROLINE SERIES
}

The Maskit embedding $\mathcal{M}$ of a surface $\Sigma$ is the space of geometrically finite groups on the boundary of quasifuchsian space for which the "top" end is homeomorphic to $\Sigma$, while the "bottom" end consists of triply punctured spheres, the remains of $\Sigma$ when a set of pants curves have been pinched. As such representations vary in the character variety, the conformal structure on the top side varies over the Teichmüller space $\mathcal{T}(\Sigma)$.

We investigate $\mathcal{M}$ when $\Sigma$ is a twice punctured torus, using the method of pleating rays. Fix a projective measure class $[\mu]$ supported on closed curves on $\Sigma$. The pleating ray $\mathcal{P}_{[\mu]}$ consists of those groups in $\mathcal{M}$ for which the bending measure of the top component of the convex hull boundary of the associated 3-manifold is in $[\mu]$. It is known that $\mathcal{P}$ is a real $1-$ submanifold of $\mathcal{M}$. Our main result is a formula for the asymptotic direction of $\mathcal{P}$ in $\mathcal{M}$ as the bending measure tends to zero, in terms of natural parameters for the complex 2-dimensional representation space $\mathcal{R}$ and the Dehn-Thurston coordinates of the support curves to $[\mu]$ relative to the pinched curves on the bottom side. This leads to a method of locating $\mathcal{M}$ in $\mathcal{R}$.

30F40; 30F60, 57M50

\section{Introduction}

Pictures of various slices and embeddings of one dimensional Teichmüller spaces into $\mathbb{C}$ have become familiar in recent years. A common feature is the complicated fractal boundary which has been studied by various authors, for example, Miyachi [24] and Mumford, Series and Wright [25]. Such examples are always based on the once punctured torus and its close relatives. This paper presents for the first time a method which makes viable the prospect of plotting a deformation space associated to a higher genus surface. The project immediately introduces many difficulties: such a deformation space will intrinsically have at least 2 complex dimensions and the underlying combinatorics of the curve complex is not that of the Farey tesselation associated to the once punctured torus.

The example we choose is the Maskit embedding of the twice punctured torus, in which the representation variety is smooth of complex dimension 2 . The key idea is explicitly 
to locate the pleating rays, that is, the loci in the representation variety along which the projective class of the bending measure of the convex hull boundary is fixed. These lines are certain branches of the solution set of a family of equations where traces of various elements in the group take real values. To explain in more detail, we first consider the analogous embedding for the once punctured torus $\Sigma_{1,1}$.

The Maskit embedding of $\Sigma_{1,1}$ was initially explored experimentally by Mumford and Wright [30; 25]. The detailed study [15] by the author and Linda Keen introduced the concept of pleating rays which justified these computational results. As explained in more detail below, these rays were used to plot Figure 1. The lined region, which repeats periodically with period 2 in both directions, indicates all representations $\rho: \pi_{1}\left(\Sigma_{1,1}\right) \rightarrow \operatorname{SL}(2, \mathbb{C})$ whose image $G$ is free and discrete and for which one fixed essential nonperipheral simple curve $\gamma_{\infty} \in \pi_{1}\left(\Sigma_{1,1}\right)$ is accidentally parabolic. The parameter $\mu \in \mathbb{C}$ is essentially the trace of another fixed curve $\gamma_{0}$ which together with $\gamma_{\infty}$ generates $\pi_{1}$. After suitable normalisation, this is enough to determine a representation $\rho$. The resulting hyperbolic 3 -manifold $\mathbb{H}^{3} / G$ is geometrically finite and homeomorphic to $\Sigma_{1,1} \times \mathbb{R}$. Its end invariants $\omega^{ \pm}$are both Riemann surfaces, representing the conformal structures on the quotients of the components of the regular set by $G$. One end $\omega^{-}$is conformally a triply punctured sphere, corresponding to the surface $\Sigma_{1,1}$ with the fixed curve $\gamma_{\infty}$ pinched. The other end $\omega^{+}$is a Riemann surface homeomorphic to $\Sigma_{1,1}$ and can thus be viewed a point in $\mathcal{T}\left(\Sigma_{1,1}\right)$. By standard Ahlfors-Bers theory, each point in $\mathcal{T}$ is represented up to conjugation by exactly one such group $G$. The Maskit embedding is the map $\mathcal{T} \rightarrow \mathbb{C}$ which takes a surface to the $\mu$-parameter of the group $G$ which represents it. In Figure 1, the parameter $i \mu=\operatorname{Tr} \gamma_{0}$ has been chosen so that the embedding is as close to the identity map $\mathcal{T}\left(\Sigma_{1,1}\right)=\mathbb{H}^{2} \hookrightarrow \mathbb{C}$ as possible. The embedding repeats periodically under translation $\mu \mapsto \mu+2$.

This paper lays the foundation for computing the analogous picture of the Maskit embedding when $\Sigma=\Sigma_{1,2}$ is a twice punctured torus. The relevant component of the representation variety $\mathcal{R}(\Sigma)$ is smooth of complex dimension 2. Thus our eventual aim is to locate the image $\mathcal{M}$ of the Maskit embedding of the Teichmüller space $\mathcal{T}(\Sigma)$ in $\mathbb{C}^{2}$. As for $\Sigma_{1,1}$, we will do this by locating the pleating rays, which in this case are real 1-submanifolds of $\mathcal{M}$ along which the projective class of the bending measure of the component $\partial \mathcal{C}^{+} / G$ of the convex hull boundary facing $\omega^{+}$is supported on a fixed pair of disjoint closed curves on $\Sigma$. In general, the pleating ray is a connected nonsingular branch of the real algebraic variety along which the traces of the support curves take real values; see Theorem 3.4. The main results of this paper identify the correct branch by determining its direction as the parameters of the representation tend to infinity, equivalently as the bending measure tends to zero; see Theorem A. The idea 


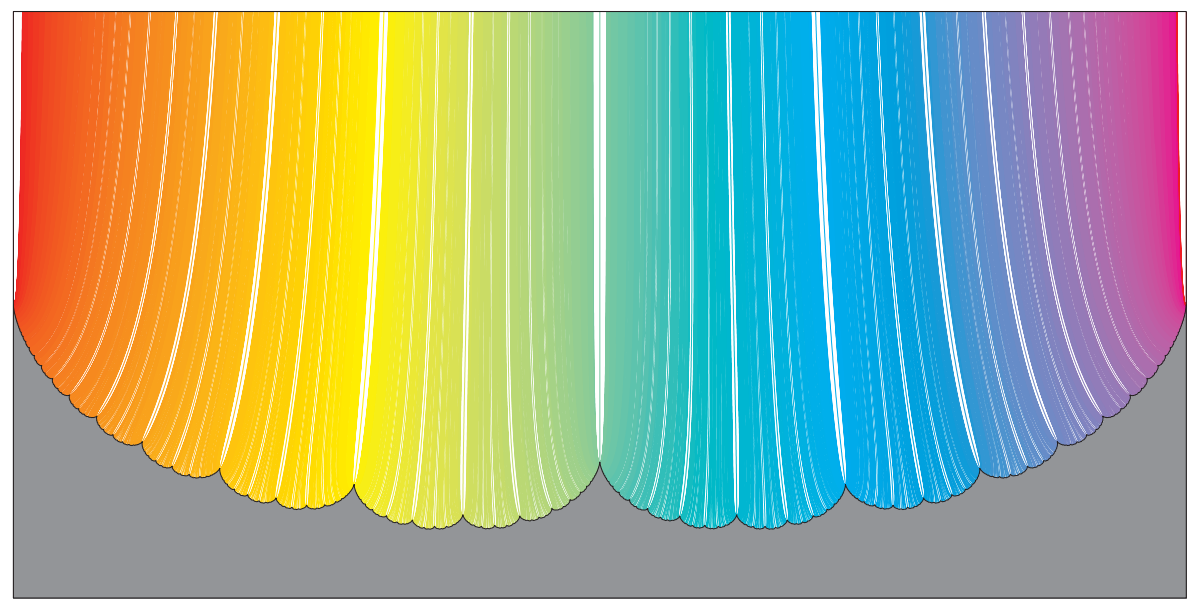

Figure 1: The Maskit embedding for the once punctured torus, showing one period in the upper half $\mu$-plane. The image of $\mathcal{T}\left(\Sigma_{1,1}\right)$ is filled by the light gray pleating rays. Picture by David Wright, reproduced from [25] with permission of Cambridge University Press.

is that $\mathcal{M}$ can then be plotted by following these real trace branches until one of the supporting curves becomes parabolic; see Section 5. A start on such a programme has been made by Austin [1].

Before stating our main theorems, we briefly review our previous results from [15]. As is well known, the simple closed curves on $\Sigma_{1,1}$ can be enumerated by the rationals $\mathbb{Q} \cup \infty$. Normalize so that the exceptional pinched curve $\gamma_{\infty}$ is represented by $\infty$. There is one ray $\mathcal{P}_{p / q}$ for each $p / q \in \mathbb{Q}$, representing all curves $\gamma_{p / q}$ whose images in $\mathcal{M}$ are loxodromic. At any point on this ray, $\partial \mathcal{C}^{+} / G$ is a pleated surface bent along $\gamma_{p / q}$ while the component $\partial \mathcal{C}^{-} / G$ facing $\omega^{-}$can be viewed as a copy of $\Sigma$ bent along $\gamma_{\infty}$ with bending angle $\pi$. If a closed curve $\gamma_{p / q}$ is a bending line, then its complex length is real, so that $\gamma_{p / q}$ is purely hyperbolic on $\mathcal{P}_{p / q}$. The trace, hence also the complex length, of $\gamma_{p / q}$ has no critical points on $\mathcal{P}_{p / q}$. It follows that $\mathcal{P}_{p / q}$ is a totally real 1 -submanifold embedded in $\mathcal{M}$ and that the hyperbolic length $l_{p / q}$ of $\gamma_{p / q}$ is strictly monotonic along $\mathcal{P}_{p / q}$ with range $(0, \infty)$. As $l_{p / q} \rightarrow 0$ along $\mathcal{P}_{p / q}$ we approach the boundary $\partial \mathcal{M}$, arriving at an algebraic limit which is the doubly cusped group in which $l_{p / q}=0$. As $l_{p / q} \rightarrow \infty$ on the other hand, there is no algebraic limit and the sequence of representations diverges. One of the main results of [15] is that $\mathcal{P}_{p / q}$ is asymptotic to the line $\Re \mu=2 p / q$ as $l_{p / q} \rightarrow \infty$, identifying it uniquely among branches of $\operatorname{Tr} \gamma_{p / q} \in \mathbb{R}$. 
Turning now to the twice punctured torus $\Sigma=\Sigma_{1,2}$, suppose we have a geometrically finite free and discrete representation for which $M=\Sigma \times \mathbb{R}$. Fix elements $S_{1}, S_{2} \in$ $\pi_{1}(\Sigma)$ corresponding to disjoint nonhomotopic closed curves $\sigma_{1}, \sigma_{2}$ which form a maximal pants decomposition of $\Sigma$ and neither of which individually disconnects $\Sigma$. We consider groups for which the conformal end $\omega^{-}$is a union of triply punctured spheres glued across punctures corresponding to $\sigma_{1}, \sigma_{2}$, while $\omega^{+}$is a marked Riemann surface homeomorphic to $\Sigma$. In Section 2.2 we give an explicit parameterisation of a holomorphic family of representations $\rho: \pi_{1}(\Sigma) \rightarrow G\left(\tau_{1}, \tau_{2}\right),\left(\tau_{1}, \tau_{2}\right) \in \mathbb{C}^{2}$, such that, for suitable values of the parameters, $G\left(\tau_{1}, \tau_{2}\right)$ has the above geometry. The Maskit embedding is the map which sends a point $X \in \mathcal{T}\left(\Sigma_{1,2}\right)$ to the point $\left(\tau_{1}, \tau_{2}\right) \in \mathbb{C}^{2}$ for which the group $G\left(\tau_{1}, \tau_{2}\right)$ has $\omega^{+}=X$. Denote the image of this map by $\mathcal{M}=\mathcal{M}\left(\Sigma_{1,2}\right)$.

Given a projective measured lamination $[\xi]$ on $\Sigma$, the pleating ray $\mathcal{P}_{[\xi]}$ is the set of groups in $\mathcal{M}$ for which the bending measure $\beta(G)$ of $\partial \mathcal{C}^{+} / G$ is in the class $[\xi]$. We restrict to pleating rays for which $[\xi]$ is rational, that is, supported on closed curves, and for simplicity write $\mathcal{P}_{\xi}$ in place of $\mathcal{P}_{[\xi]}$, although noting that $\mathcal{P}_{\xi}$ depends only on $[\xi]$. From general results of Bonahon and Otal [5] (see Theorem 3.1 in Section 3), for any pants decomposition $\gamma_{1}, \gamma_{2}$ such that $\sigma_{1}, \sigma_{2}, \gamma_{1}, \gamma_{2}$ are mutually nonhomotopic and fill up $\Sigma$, and any pair of angles $\theta_{i} \in(0, \pi)$, there is a unique group in $\mathcal{M}$ for which the bending measure of $\partial \mathcal{C}^{+} / G$ is $\sum_{1,2} \theta_{i} \delta_{\gamma_{i}}$. (This extends to the case $\theta_{i}=0$ provided $\sigma_{1}, \sigma_{2}, \gamma_{j}$ fill up $\Sigma$ (see Section 3), also to the case $\theta=\pi$.) Thus given $\xi=\sum_{1,2} a_{i} \delta_{\gamma_{i}}$, there is a unique group $G=G_{\xi}(\theta) \in \mathcal{M}$ with bending measure $\beta(G)=\theta \xi$ for any sufficiently small $\theta>0$.

In contrast to the quasifuchsian situation studied by the author in [28], there is no algebraic limit along $\mathcal{P}_{\xi}$ as $\theta \rightarrow 0$; see Corollary 6.5. Intuitively this is because the groups $G_{\xi}(\theta)$ want to limit on a Fuchsian group, which is however impossible since the bending angles on the parabolic pinched curves are fixed as $\pi$. Our main result is a formula for the asymptotic direction of $\mathcal{P}_{\xi}$ in $\mathcal{M} \subset \mathbb{C}^{2}$ in terms of the global linear coordinates for measured laminations on $\Sigma_{2,1}$ set up by Keen, Parker and the author [14]. These coordinates, called here canonical coordinates, assign to a measured lamination $\xi$ a point $\mathbf{i}(\xi)=\left(q_{1}(\xi), p_{1}(\xi), q_{2}(\xi), p_{2}(\xi)\right) \in\left(\mathbb{R}_{+} \times \mathbb{R}\right)^{2}$; see Section 4. (For the extension of these coordinates to arbitrary surfaces, see Maloni and Series [18].) The coordinates of a simple closed curve are integral; they are essentially its Dehn-Thurston coordinates relative to $\sigma_{1}, \sigma_{2}$ and are a close analogue of the $p, q$ coordinates for curves on $\Sigma_{1,1}$ above. In particular, $q_{i}(\gamma)=i\left(\gamma, \sigma_{i}\right)$ where $i(\cdot, \cdot)$ is the usual geometric intersection number. If $\xi=\sum_{1,2} a_{i} \delta_{\gamma_{i}}$, the above Bonahon-Otal condition on $\sigma_{1}, \sigma_{2}, \gamma_{1}, \gamma_{2}$ is equivalent to $q_{i}(\xi)>0, i=1,2$. We call such laminations admissible; see Section 3. We call a pair of curves $\gamma_{1}, \gamma_{2}$ 
exceptional if $q_{1}\left(\gamma_{1}\right) q_{2}\left(\gamma_{2}\right)=q_{1}\left(\gamma_{2}\right) q_{2}\left(\gamma_{1}\right)$, and we say $\xi=\sum_{1,2} a_{i} \delta_{\gamma_{i}}$ is exceptional if $a_{i}>0, i=1,2$ and the pair $\gamma_{1}, \gamma_{2}$ is exceptional. The main result of this paper is:

Theorem A Suppose that $\xi=\sum_{1,2} a_{i} \delta_{\gamma_{i}}$ is admissible and not exceptional. Then as the bending measure $\beta(G) \in \mathbb{R}^{+} \xi$ tends to zero, the pleating ray $\mathcal{P}_{\xi}$ approaches the line

$$
\Re \tau_{i}=2 p_{i}(\xi) / q_{i}(\xi), \quad \operatorname{Arg} \tau_{i}=\pi / 2, \quad \Im \tau_{1} / \Im \tau_{2}=q_{2}(\xi) / q_{1}(\xi)
$$

This theorem is stated more precisely as Theorem 7.3. To actually locate the pleating rays, note (Lemma 3.3) that $\operatorname{Tr} \rho(\gamma)$ is real whenever $\gamma$ is a bending line. We prove:

Theorem B Suppose that $\xi=\sum_{1,2} a_{i} \delta_{\gamma_{i}}$ is admissible and that the pair $\gamma_{1}, \gamma_{2}$ is not exceptional. Then any point on the ray $\mathcal{P}_{\xi}$ satisfies the equations $\mathfrak{\Im} \operatorname{Tr} \rho\left(\gamma_{i}\right)=0, i=1,2$ and these equations have a unique solution as $\tau_{i} \rightarrow \infty$ in the direction specified by Theorem A. If the curve $\gamma_{1}$ is admissible, then there exists $\gamma_{2}$ disjoint from $\gamma_{1}$ such that the pair $\gamma_{1}, \gamma_{2}$ is not exceptional, and thus $\mathcal{P}_{\gamma_{1}}$ is determined by the above result applied to $\xi=1 \cdot \delta_{\gamma_{1}}+0 \cdot \delta_{\gamma_{2}}$.

In the exceptional case we obtain only partial results detailed in Theorem 7.5. We believe the above theorems to be still true in this case, but as discussed in Section 7.2, the result appears to be beyond the scope of this paper. The lack of a complete result will not affect the plotting of the asymptotic arrangement of pleating rays and planes.

As explained in Section 5, these results in principle enable one to compute $\mathcal{M}$, modulo the unproven conjecture that the rational pleating rays are dense. We hope to explore how to actually implement the computations in practice elsewhere. ${ }^{1}$

Theorems A and B are proved together. The proofs have two main parts. First (Section 6) we show that asymptotically, the lengths of the geodesic representatives $\sigma_{1}^{+}, \sigma_{2}^{+}$of $\sigma_{1}, \sigma_{2}$ on $\partial \mathcal{C}^{+} / G$ tend to 0 while at the same time becoming orthogonal to the bending lines. (This should be compared to the situation in [28], where in the limit as the bending angles go to zero, the bending lines on $\partial \mathcal{C}^{+} / G$ and $\partial \mathcal{C}^{-} / G$ become "orthogonal" in the sense that average of the cosine of the angle between them goes to zero.) From this we deduce (Theorem 6.1) that as $\theta \rightarrow 0, \tau_{1}, \tau_{2} \rightarrow \infty$ in such a way that

$$
\operatorname{Arg} \tau_{i} \rightarrow \pi / 2, \quad \Im \tau_{1} / \Im \tau_{2} \rightarrow q_{2}(\xi) / q_{1}(\xi)
$$

${ }^{1}$ Since this paper was written, A Austin has used Theorem A to compute "wheels" of rays which correspond to all rational laminations in the link of a given curve; see Austin [1] and the forthcoming paper of Austin with the author [2]. 
Second, we use a formula for trace polynomials from [14]. Note that the trace $\operatorname{Tr} \rho(\gamma)$ is a polynomial on the parameter space $\mathbb{C}^{2}$. The formula (see Theorem 4.1) expresses the top terms of this polynomial in terms of its canonical coordinates $\mathbf{i}(\gamma)$. We also make use Thurston's symplectic form on the space of measured laminations $M L$, which turns out to have the standard form relative to our canonical coordinates (Section 4.2). To complete the proofs of Theorems A and B, in Section 7 we use the asymptotics of the trace polynomials together with Thurston's form and some simple linear algebra to extract the unique possible asymptotic directions of the pleating rays.

One might also ask for the limit of the hyperbolic structure on $\partial \mathcal{C}^{+} / G$ as the bending measure tends to zero. The following result is an immediate consequence of the first part of the proof of Theorem A:

Theorem C Let $\xi=\sum_{1,2} a_{i} \delta_{\gamma_{i}}$ be as above. Then as the bending measure $\beta(G) \in$ $\mathbb{R}^{+} \xi$ tends to zero, the induced hyperbolic structure of $\partial \mathcal{C}^{+} / G$ along $\mathcal{P}_{\xi}$ converges to the barycentre of the laminations $\sigma_{1}$ and $\sigma_{2}$ in the Thurston boundary of $\mathcal{T}(\Sigma)$.

This should be compared with the result in [28], that the analogous limit through groups whose bending laminations on the two sides of the convex hull boundary are in the classes of a fixed pair of laminations $\left[\xi^{ \pm}\right]$, is a Fuchsian group on the line of minima of $\left[\xi^{ \pm}\right]$.

Although this paper is written in the context of the twice punctured torus, the results of Section 6 and hence also Theorem $C$ should apply to the Maskit embedding of a general surface. The top terms formula is needed only to determine the asymptotic value of $\Re \tau_{i}$. Y Chiang obtained an analogous top terms formula for the five times punctured sphere in [7]. We believe there is a more general result and hope to explore this elsewhere. ${ }^{2}$

The plan of the paper is as follows. In Section 2 we describe our holomorphic family of groups which realise the Maskit embedding and give estimates on the rough shape of $\mathcal{M}$. In Section 3 we briefly review facts about convex hull boundaries, bending measures and pleating rays. In Section 4 we review canonical coordinates for simple curves and the top terms formula from [14], and discuss Thurston's symplectic form. In Section 5 we discuss in more detail how Theorem A may be used to compute pleating rays and illustrate the theorem with some very simple examples which can be computed by hand. The remaining two sections contain the main work of the paper as described above. Theorem $\mathrm{C}$ is proved at the end of Section 6 and Theorems A and B are proved in Section 7.

\footnotetext{
${ }^{2}$ Since this paper was written, an analogous top terms formula for a general hyperbolisable surface has indeed been proved by S Maloni and the author [18].
} 
Acknowledgments This paper was begun in the early 1990's as joint project with Linda Keen and John Parker. I would like to thank them for allowing me to include some of our preliminary results and to continue alone. Most of our joint results are contained in [14]; other work done in draft only we refer to here as [13]. We conjectured a partial version of Theorem A but proofs were incomplete - in particular we lacked the orthogonality idea, the use of Thurston's symplectic form, the use of Minsky's twist and the major general results in $[5 ; 9]$.

I would like to thank MSRI and the organisers of the program on Teichmüller space and Kleinian groups for their hospitality: during the program the research for this paper was completed.

\section{The Maskit embedding and plumbing parameters}

Let $\Sigma=\Sigma_{1,2}$ be a twice punctured torus. Figure 2 shows a fundamental domain $\Delta$ for a Fuchsian representation of $\Sigma$, on which some definite hyperbolic metric has been fixed. The sides of $\Delta$ are identified by hyperbolic isometries $S_{1}, S_{2}, T$ which we can view as free generators for $\pi_{1}(\Sigma)$.

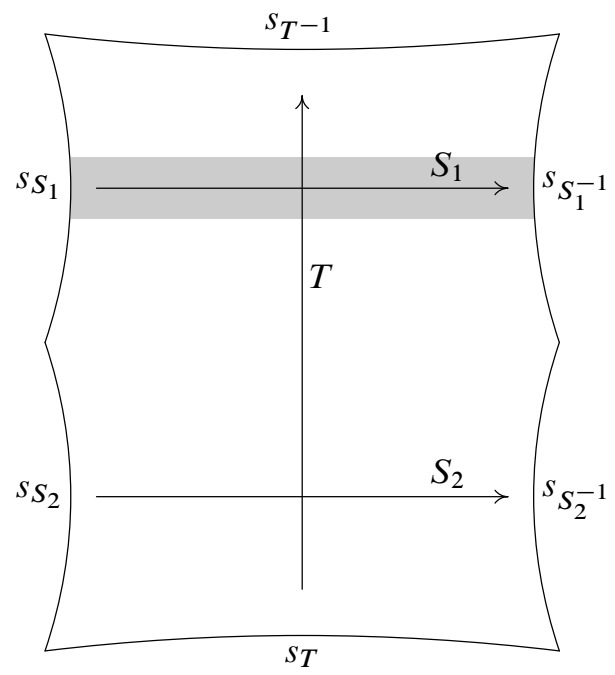

Figure 2: The fundamental domain $\Delta$

\subsection{The Maskit embedding}

Let $\mathcal{R}(\Sigma)$ be the representation variety of $\pi_{1}(\Sigma)$, that is, the set of representations $\rho: \pi_{1}\left(\Sigma_{1,2}\right) \rightarrow \operatorname{SL}(2, \mathbb{C})$ modulo conjugation in $\operatorname{SL}(2, \mathbb{C})$ (but see for example 
Kapovich [11, page 61]) with the algebraic topology. Let $\mathcal{M} \subset \mathcal{R}$ be the subset of representations $\rho$ for which:

(i) The image $G=\rho\left(\pi_{1}\left(\Sigma_{1,2}\right)\right)$ is free and discrete and the images of the loops around the two punctures $S_{1} S_{2}^{-1}$ and $T S_{2}^{-1} T^{-1} S_{1}$ are parabolic.

(ii) The images of $S_{i}, i=1,2$ are parabolic.

(iii) All components of the regular set $\Omega(G)$ are simply connected and there is exactly one invariant component $\Omega^{+}(G)$.

(iv) The quotient $\Omega(G) / G$ has 3 components; $\Omega^{+}(G) / G$ is homeomorphic to $\Sigma$ and the other two components are triply punctured spheres.

In this situation (see for example Marden [19, Section 3.8]), the corresponding 3manifold $M_{\rho}=\mathbb{H}^{3} / G$ is topologically $\Sigma_{1,2} \times(0,1)$. Such a group $G$ is a geometrically finite cusp group on the boundary (in the algebraic topology) of the set of quasifuchsian representations of $\pi_{1}(\Sigma)$. The "top" component $\Omega^{+} / G$ of the conformal boundary may be identified to $\Sigma \times\{1\}$ and is homeomorphic to $\Sigma$. On the "bottom" component $\Omega^{-} / G$, identified to $\Sigma \times\{0\}$, the two curves $\sigma_{1}, \sigma_{2}$ corresponding to the generators $S_{1}, S_{2}$ have been pinched, making $\Omega^{-} / G$ a union of two triply punctured spheres. The conformal structure on $\Omega^{+} / G$, together with the pinched curves $\sigma_{1}, \sigma_{2}$, are the end invariants of $M$ in the sense of Minsky's ending lamination theorem. Since a triply punctured sphere is rigid, the conformal structure on $\Omega^{-} / G$ is fixed independent of $\rho$. The structure on $\Omega^{+} / G$ varies; it follows from standard Ahlfors-Bers theory using the measurable Riemann mapping theorem (see again Marden [19, Section 3.8]), that there is a unique group corresponding to each possible conformal structure on $\Omega^{+} / G$. Formally, the Maskit embedding of the Teichmüller space of $\Sigma$ is the map

$$
\Phi: \mathcal{T}(\Sigma) \rightarrow \mathbb{C}^{2}
$$

which sends a point $X \in \mathcal{T}(\Sigma)$ to the unique group $G \in \mathcal{M}$ for which $\Omega^{+} / G$ has the marked conformal structure $X$. By abuse of terminology, we also refer to $\mathcal{M}$ as the Maskit embedding or Maskit slice of $\Sigma_{1,2}$.

\subsection{A concrete realisation of $\mathcal{M}$}

Groups in the Maskit slice $\mathcal{M}$ may be manufactured by the plumbing construction of Kra [17]; see Section 2.3 below. Here we simply write down a suitable holomorphic family of representations and verify directly that groups thus constructed have the required properties. Groups in the family depend on two complex parameters $\tau_{1}, \tau_{2} \in \mathbb{C}$. 
To define $\rho: \pi_{1}\left(\Sigma_{1,2}\right) \rightarrow \operatorname{SL}(2, \mathbb{C})$, it suffices to give the images of the three free generators $S_{1}, S_{2}, T$ of $\pi_{1}(\Sigma)$. Following [14], for $\tau_{1}, \tau_{2} \in \mathbb{C}$ define $\rho=\rho\left(\tau_{1}, \tau_{2}\right)$ by

$$
\rho\left(S_{1}\right)=\left(\begin{array}{ll}
1 & 2 \\
0 & 1
\end{array}\right), \quad \rho\left(S_{2}\right)=\left(\begin{array}{ll}
1 & 0 \\
2 & 1
\end{array}\right), \quad \rho(T)=\left(\begin{array}{cc}
1+\tau_{1} \tau_{2} & \tau_{1} \\
\tau_{2} & 1
\end{array}\right) .
$$

Denote the image of $\rho\left(\tau_{1}, \tau_{2}\right)$ by $G\left(\tau_{1}, \tau_{2}\right)$. Note that the holomorphic family $\mathcal{G}=$ $\left\{G\left(\tau_{1}, \tau_{2}\right): \tau_{i} \in \mathbb{C}\right\}$ has complex dimension 2 , the dimension of Teich $(\Sigma)$. Not all groups $\mathcal{G}$ lie in $\mathcal{M}$, in particular any representation with $\Im \tau_{i}=0, i=1,2$ is Fuchsian and so not in $\mathcal{M}$. We also need to restrict $\tau_{1}, \tau_{2}$ so as to have only one copy of each group up to conjugation. By abuse of notation, for $W \in \pi_{1}(\Sigma)$, we shall use $W$ also to denote the image $\rho(W) \in G\left(\tau_{1}, \tau_{2}\right)$, and write $W=W\left(\tau_{1}, \tau_{2}\right)$ as needed to avoid confusion. By direct computation (see Appendix 1) we find $\operatorname{Tr}\left[S_{i}, T^{-1}\right]=\tau_{j}^{2}+2$ where $j=1+i \bmod 2$. Thus $\tau_{1}^{2}, \tau_{2}^{2}$ are invariant functions on $\mathcal{R}$, so that the component $\mathbb{C}_{+}^{2}$ of $\mathbb{C}^{2} \backslash\left\{\Im \tau_{i}=0\right\}$ with $\Im \tau_{i}>0, i=1,2$, consists entirely of nonconjugate groups.

The following propositions from [13] justify our use of the family $\mathcal{G}$.

Proposition 2.1 Let $G\left(\tau_{1}, \tau_{2}\right) \in \mathcal{G}$ be as above. If $\Im \tau_{i}>1, i=1,2$ and $\Im \tau_{1} \Im \tau_{2}>4$ then $G\left(\tau_{1}, \tau_{2}\right) \in \mathcal{M}$. Moreover the limit set $\Lambda(G)$ is contained in the two strips $0 \leq \mathfrak{I} z \leq 1 / 2, \mathfrak{\Im} \tau_{1}-1 / 2 \leq \mathfrak{\Im} z \leq \mathfrak{\Im} \tau_{1}$, together with the point at $\infty$.

Proof A fundamental domain for $G=G\left(\tau_{1}, \tau_{2}\right)$ is shown in Figure 3, in which the disks $B_{2}, B_{3}$ have equal radius $1 / \Im \tau_{2}$. The formal proof that $G$ is free and discrete is a straightforward application of Maskit's second combination theorem [20, page 160]. To see that $G \in \mathcal{M}$, one checks from the proof of the combination theorem that the lower half plane and the half plane above the line $\mathfrak{\Im} z=\Im \tau_{1}$ project to the two triply punctured spheres which together form $\Omega^{-} / G$, while the simply connected component $\Omega^{+}$is contained in the strip $0<\mathfrak{I} z<\mathfrak{\Im} \tau_{1}$. The claim about $\Lambda$ also follows. For further details, see Appendix 1.

As explained above, a standard argument using the measurable Riemann mapping theorem now shows that any group in $\mathcal{M}$ can be represented by a group in $\mathcal{G}$. Complementing Proposition 2.1 we have the following result, also proved in Appendix 1.

Proposition 2.2 Suppose that $G\left(\tau_{1}, \tau_{2}\right) \in \mathcal{M}$. Then $\Im \tau_{i} \geq 1 / 2, i=1,2$ and $\mathfrak{\Im} \tau_{1} \Im \tau_{2} \geq 1$.

We shall mainly do our calculations with $G$ normalised as above, so that $S_{1}(z)=z+2$. On occasion it is convenient to normalise $S_{2}$ in this way, in which case we interchange the roles of $\tau_{1}$ and $\tau_{2}$. More precisely we have: 


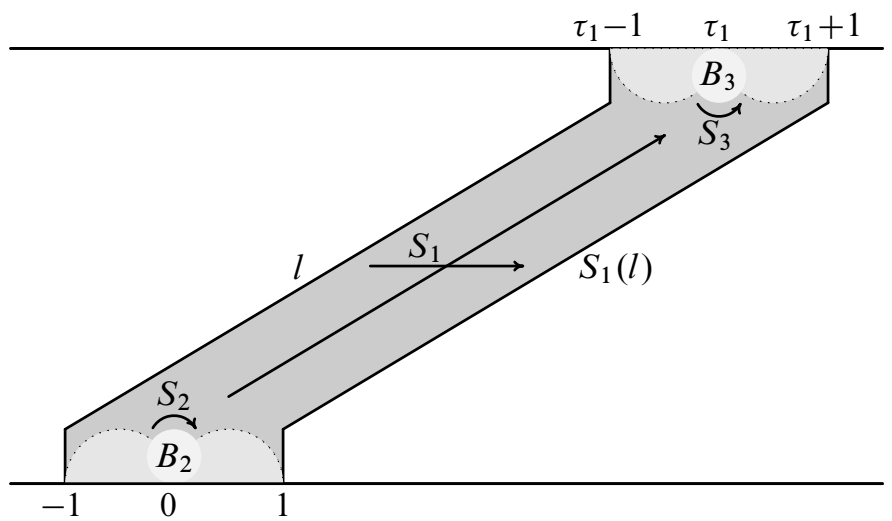

Figure 3: A fundamental domain for $G\left(\tau_{1}, \tau_{2}\right)$

Lemma 2.3 The involution $K(z)=-1 / \bar{z}$ conjugates $S_{1}$ to $S_{2}$. Moreover,

$$
K T\left(\tau_{1}, \tau_{2}\right) K^{-1}=T\left(-\bar{\tau}_{2},-\bar{\tau}_{1}\right)^{-1} .
$$

To get some further feeling for $\mathcal{M}$, note that the right Dehn twist $D_{\sigma_{1}}$ around $\sigma_{1}$ induces the automorphism $S_{1} \mapsto S_{1}, S_{2} \mapsto S_{2}$ and $T \mapsto S_{1} T$ of $\pi_{1}(\Sigma)$. Since $S_{1} T\left(\tau_{1}, \tau_{2}\right)=T\left(\tau_{1}+2, \tau_{2}\right)$, it follows that $D_{\sigma_{1}}$ induces the map $\left(\tau_{1}, \tau_{2}\right) \mapsto\left(\tau_{1}+2, \tau_{2}\right)$ on $\mathcal{M}$, and similarly for $D_{\sigma_{2}}$.

By abuse of notation, from now on we use $\mathcal{M}$ to denote the set of $\left(\tau_{1}, \tau_{2}\right)$ with $\Im \tau_{i}>0$ and for which the group $G\left(\tau_{1}, \tau_{2}\right)$ has properties (i), (ii) and (iii) above. The above two propositions give rough bounds on the shape of $\mathcal{M}$.

\subsection{The plumbing construction}

The parameters $\tau_{i}$ have geometrical meaning as the plumbing parameters of Kra [17]. The idea is to make a projective structure on $\Sigma$ by starting from two triply punctured spheres with punctures identified in pairs. One "plumbs" across the punctures by identifying punctured disk neighbourhoods $D, D^{\prime}$ of the two paired cusps using the formula $z w=\tau$ where $z, w$ are holomorphic parameters in $D$ and $D^{\prime}$. In the hyperbolic metric, this corresponds to identifying the punctured disks by twisting by $\Re \tau$ and scaling by a factor of $\Im \tau$. Making this construction in the above setting, with plumbing parameter $\tau_{i}$ across the puncture corresponding to $\sigma_{i}$, results in the family $\mathcal{G}$. For a more detailed and general account of plumbing, see also Maloni and Series [18]. 


\section{Bending lines and pleating rays}

Let $M=\mathbb{H}^{3} / G$ be a hyperbolic 3-manifold, and let $\mathcal{C} / G$ be its convex core, where $\mathcal{C}$ is the convex hull in $\mathbb{H}^{3}$ of the limit set of $G$; see Epstein and Marden [10]. If $M$ is geometrically finite then there is a natural homeomorphism between each component of $\partial \mathcal{C} / G$ and of $\Omega / G$. Each component $F$ of $\partial \mathcal{C} / G$ inherits an induced hyperbolic structure from $M$. Moreover $F$ is a pleated surface, meaning that it is the isometric image under a map $f: H \rightarrow M$ of a hyperbolic surface $H$ which restricts to an isometry into $M$ on the leaves of a geodesic lamination $L$ on $H$, and which is also an isometry on each complementary component of $L$. (Strictly, the pleated surface is the pair $(H, f)$; this becomes important when considering the induced marking on $F$.) We call $L$ the bending lamination of $F$ and the images of the complementary components of $L$, the flat pieces of $F$. The bending lamination carries a transverse measure called the bending measure which describes the angles between the flat pieces; see Epstein and Marden [10] for details. By a bending line of $F$, we will mean any complete geodesic on $\partial \mathcal{C} / G$ which is either completely contained in $L$, or in the interior of a flat part. We also use the term bending line to mean any lift of a bending line of $F$ to a complete geodesic in $\mathbb{H}^{3}$.

We shall be interested in manifolds for which the bending lamination is rational, that is, supported on closed curves. Denote the space of homotopy classes of simple closed essential loops on $F$ by $\mathcal{S}(F)$ and the space of measured laminations on $F$ by $M L(F)$. The subset of rational laminations is denoted $M L_{\mathbb{Q}}$ and consists of measured laminations of the form $\sum_{i} a_{i} \delta_{\gamma_{i}}$, abbreviated $\sum_{i} a_{i} \gamma_{i}$, where the curves $\gamma_{i} \in \mathcal{S}(F)$ are disjoint and nonhomotopic, $a_{i} \geq 0$, and $\delta_{\gamma_{i}}$ denotes the transverse measure which gives weight 1 to each intersection with $\gamma_{i}$. If $\sum_{i} a_{i} \gamma_{i}$ is the bending measure of a pleated surface $F$, then $a_{i}$ is the angle between the flat pieces adjacent to $\gamma_{i}$, also denoted $\theta_{\gamma_{i}}$. In particular, $\theta_{\gamma_{i}}=0$ if and only if the flat pieces adjacent to $\gamma_{i}$ are in a common totally geodesic subset of $\partial \mathcal{C} / G$, equivalently have lifts which lie in the same hyperbolic plane in $\mathbb{H}^{3}$.

We take the term pleated surface to include the case in which a closed leaf $\gamma$ of the bending lamination maps to the fixed point of a rank one parabolic cusp of $M$. In this case, the image pleated surface is cut along $\gamma$ and thus may be disconnected. Moreover the bending angle between the flat pieces adjacent to $\gamma$ is $\pi$. This is because for a geometrically finite group, the punctures on the two components of $\partial \mathcal{C} / G$ are paired and the associated flat pieces lift to two tangent half planes in $\mathbb{H}^{3}$; see eg Marden [19, Chapter 3] for details. 
The key results about the existence of hyperbolic manifolds with prescribed bending laminations are due to Bonahon and Otal. We need the following special case of their main theorem:

Theorem 3.1 [5, Theorem 1] Let $X$ be a hyperbolisable surface, possibly with punctures, and let $M$ be a 3-manifold homeomorphic to $X \times(0,1)$. Suppose also that $\xi^{ \pm}=\sum_{i} a_{i}^{ \pm} \gamma_{i}^{ \pm}$are rational measured laminations on $X$. Then there exists a geometrically finite group $G$ such that $M=\mathbb{H}^{3} / G$ and such that the bending measures on the two components $\partial \mathcal{C}^{ \pm} / G$ of the convex hull boundary $\partial \mathcal{C} / G$ equal $\xi^{ \pm}$ respectively, if and only if $a_{i}^{ \pm} \in(0, \pi]$ for all $i$ and $\left\{\gamma_{i}^{ \pm}, i=1, \ldots, n\right\}$ fill up $X$, equivalently if $i\left(\xi^{+}, \gamma\right)+i\left(\xi^{-}, \gamma\right)>0$ for every essential simple closed curve $\gamma \in$ $\mathcal{S}(X)$. If such a structure exists, it is unique.

Specialising now to the case of interest to this paper, let $\rho=\rho\left(\tau_{1}, \tau_{2}\right)$ be a representation $\pi_{1}\left(\Sigma_{1,2}\right) \rightarrow \operatorname{SL}(2, \mathbb{C})$ in the family $\mathcal{G}$ from Section 2, and suppose that the image $G=$ $G\left(\tau_{1}, \tau_{2}\right)$ lies in the Maskit slice $\mathcal{M}$. The boundary of the convex core $\mathcal{C} / G$ has three components, one $\partial \mathcal{C}^{+} / G$ facing $\Omega^{+} / G$ and homeomorphic to $\Sigma_{1,2}$, and two triply punctured spheres whose union we denote $\partial \mathcal{C}^{-} / G$. The induced hyperbolic structures on the two components of $\partial \mathcal{C}^{-} / G$ are rigid, while the structure on $\partial \mathcal{C}^{+} / G$ varies. We denote the bending lamination of $\partial \mathcal{C}^{+} / G$ by $\beta(G) \in M L\left(\Sigma_{1,2}\right)$. Following the discussion above, we view $\partial \mathcal{C}^{-} / G$ as a single pleated surface with bending lamination $\pi\left(\sigma_{1}+\sigma_{2}\right)$, indicating that two triply punctured spheres are glued across the annuli whose core curves $\sigma_{1}$ and $\sigma_{2}$ correspond to the parabolics $S_{i} \in G$.

Corollary 3.2 A rational measured lamination $\xi \in M L_{\mathbb{Q}}\left(\Sigma_{1,2}\right)$ is the bending measure of a group $G \in \mathcal{M}$ if and only if $i\left(\xi, \sigma_{1}\right), i\left(\xi, \sigma_{2}\right)>0$. If such a structure exists, it is unique.

We call $\xi \in M L_{\mathbb{Q}}\left(\Sigma_{1,2}\right)$ for which $i\left(\xi, \sigma_{1}\right), i\left(\xi, \sigma_{2}\right)>0$, admissible.

\subsection{Pleating rays}

Denote the set of projective measured laminations on $\Sigma_{1,2}$ by $P M L$ and the projective class of $\xi=a_{1} \gamma_{1}+a_{2} \gamma_{2} \in M L$ by $[\xi]$. The pleating ray $\mathcal{P}=\mathcal{P}_{[\xi]}$ of $\xi \in M L$ is the set of groups $G \in \mathcal{M}$ for which $\beta(G) \in[\xi]$. To simplify notation we write $\mathcal{P}_{\xi}$ for $\mathcal{P}_{[\xi]}$ and note that $\mathcal{P}_{\xi}$ depends only on the projective class of $\xi$, also that $\mathcal{P}_{\xi}$ is nonempty if and only if $\xi$ is admissible. In particular, we write $\mathcal{P}_{\gamma}$ for the ray $\mathcal{P}_{\left[\delta_{\gamma}\right]}$. As is shown by Choi and the author [9] (see also Bonahon and Otal [5]), as $\beta(G)$ increases, $\mathcal{P}_{\xi}$ eventually limits on a geometrically finite group $G_{\text {cusp }}(\xi)$ in the algebraic closure $\overrightarrow{\mathcal{M}}$ 
of $\mathcal{M}$ at which at least one of the support curves to $\xi$ is parabolic. More precisely, $\beta(G)$ increases until the value $\beta(G)=\theta\left(a_{1} \gamma_{1}+a_{2} \gamma_{2}\right)$ for which $\max \left\{\theta a_{1}, \theta \alpha_{2}\right\}=\pi$. If $\theta a_{i}=\pi$, we are at a point on $\partial \mathcal{M}$ at which the curve $\xi_{i}$ is parabolic. We write $\overline{\mathcal{P}}_{\xi}=\mathcal{P}_{\xi} \cup G_{\text {cusp }}(\xi)$.

Likewise for disjoint nonhomotopic curves $\gamma_{1}, \gamma_{2} \in \mathcal{S}$, we define the pleating plane $\mathcal{P}_{\gamma_{1}, \gamma_{2}}$ of $\gamma_{1}, \gamma_{2}$ to be the set of groups $G \in \mathcal{M}$ for which $\beta(G)=\sum a_{i} \gamma_{i}$ with $a_{i}>0$. Thus $\mathcal{P}_{\gamma_{1}, \gamma_{2}}$ is the union of the pleating rays $\mathcal{P}_{\xi}$ with $\xi=\sum a_{i} \gamma_{i}, a_{i}>0$. The rays $\mathcal{P}_{\gamma_{1}}, \mathcal{P}_{\gamma_{2}}$ are clearly contained in the boundary of $\mathcal{P}_{\gamma_{1}, \gamma_{2}}$; note that $\gamma_{i}$ may not be admissible even though $\xi=\sum a_{i} \gamma_{i}$ is. We call planes for which one or other of the support curves is not admissible degenerate, as they do not contain the corresponding ray $\mathcal{P}_{\gamma_{i}}$. We write $\overline{\mathcal{P}}_{\gamma_{1}, \gamma_{2}}=\bigcup_{\eta} \overline{\mathcal{P}}_{\eta}$ where the union is over $\eta=\sum a_{i} \gamma_{i}$ with $a_{1}, a_{2} \geq 0$.

The following key lemma is proved in [9, Proposition 4.1]; see also Lemma 4.6 of [16]. The essence is that because the two flat pieces of $\partial \mathcal{C} / G$ on either side of a bending line are invariant under translation along the bending line, the translation can have no rotational part.

Lemma 3.3 If the axis of $g \in G$ is a bending line of $\partial \mathcal{C} / G$, then $\operatorname{Tr}(g) \in \mathbb{R}$.

Notice that the lemma applies even when the bending angle $\theta_{\gamma}$ along $\gamma$ vanishes. Thus if $G \in \overline{\mathcal{P}}_{\gamma_{1}, \gamma_{2}}$ we have $\operatorname{Tr} g \in \mathbb{R}, i=1,2$, for any $g \in G$ whose axis projects to either curve $\gamma_{i}$.

In order to compute pleating planes, we need the following result which is a special case of Theorems B and C of Choi and Series [9]; see also Keen and Series [15]. Also recall that a codimension- $p$ submanifold $N \hookrightarrow \mathbb{C}^{n}$ is called totally real if it is defined locally by equations $\mathfrak{\Im} f_{i}=0, i=1, \ldots, p$, where $f_{i}, i=1, \ldots, n$ are local holomorphic coordinates for $\mathbb{C}^{n}$. As usual, if $\gamma$ is a bending line we denote its bending angle by $\theta_{\gamma}$. Recall that the complex length $\mathrm{cl}(\mathrm{A})$ of a loxodromic element $A \in \mathrm{SL}(2, \mathbb{C})$ is defined by $\operatorname{Tr} A=2 \cosh \operatorname{cl}(A) / 2$; see eg Series [27] or Choi and Series [9] for details. By construction, $\mathcal{P}_{\gamma_{1}, \gamma_{2}} \subset \mathcal{M} \subset \mathcal{R}(\Sigma)$.

Theorem 3.4 The complex lengths $c l \gamma_{1}, c l \gamma_{2}$ are local holomorphic coordinates for $\mathcal{R}(\Sigma)$ in a neighbourhood of $\mathcal{P}_{\gamma_{1}, \gamma_{2}}$. Moreover $\mathcal{P}_{\gamma_{1}, \gamma_{2}}$ is connected and is locally defined as the totally real submanifold $\Im \operatorname{Tr} \gamma_{i}=0, i=1,2$ of $\mathcal{R}$. Any pair $\left(f_{1}, f_{2}\right)$, where $f_{i}$ is either the hyperbolic length $\Re \mathrm{cl}\left(\gamma_{i}\right)$ or the bending angle $\theta_{\gamma_{i}}$, are global coordinates on $\mathcal{P}_{\gamma_{1}, \gamma_{2}}$.

This result extends to $\overline{\mathcal{P}}_{\gamma_{1}, \gamma_{2}}$, except that one has to replace $\Re \operatorname{cl}\left(\gamma_{i}\right)$ by $\operatorname{Tr} \gamma_{i}$ in a neighbourhood of a point for which $\gamma_{i}$ is parabolic. In fact as discussed in [9, Section 3.1], 
complex length and traces are interchangeable except at cusps (where traces must be used) and points where a bending angle vanishes (where complex length must be used). The parameterisation by lengths or angles extends to $\overline{\mathcal{P}}_{\gamma_{1}, \gamma_{2}}$.

Notice that the above theorem gives a local characterisation $\overline{\mathcal{P}}_{\gamma_{1}, \gamma_{2}}$ as a subset of the representation variety $\mathcal{R}$ and not just of $\mathcal{M}$. In other words, to locate $\mathcal{P}$, one does not need to check whether nearby points lie a priori in $\mathcal{M}$; it is enough to check that the traces remain real and away from 2 and that the bending angle on one or other of $\theta_{\gamma_{i}}$ does not vanish. As we shall see, this last condition can easily be checked by requiring that further traces be real valued.

\section{Canonical coordinates for simple curves}

Our main result Theorem A involves the explicit coordinatisation of the space $M L=$ $\operatorname{ML}\left(\Sigma_{1,2}\right)$ of measured laminations on $\Sigma_{1,2}$ introduced in [14]. The coordinates, called $\pi_{1,2}$-coordinates in [14] and canonical coordinates in this paper, are essentially DehnThurston coordinates relative to the curves $\sigma_{1}, \sigma_{2}$. They are global coordinates for $M L$ which take values in $\left(\mathbb{R}_{+} \times \mathbb{R}\right)^{2}$, the sign of a given coordinate in a given chart being constant. At the same time, they can be viewed as giving a piecewise linear cone structure to $M L$, the charts being laminations supported on a particular set of train tracks on $\Sigma$. Modulo their boundaries, these charts partition $M L$; which chart supports a given lamination being determined by simple linear inequalities between their coordinates. The coordinates are set up so as to maintain as close an analogy as possible with the once punctured torus $\Sigma_{1,1}$. The charts are also very closely related to the $\pi_{1}$-train tracks of [4]. Canonical coordinates are generalised to an arbitrary hyperbolisable surface in [18], where $p_{i}$ is identified precisely as the twist coordinate defined in [29].

In more detail, the canonical coordinates

$$
\mathbf{i}(\gamma)=\left(q_{1}(\gamma), p_{1}(\gamma), q_{2}(\gamma), p_{2}(\gamma)\right) \in\left(\mathbb{Z}_{+} \times \mathbb{Z}\right)^{2}
$$

of a curve $\gamma \in \mathcal{S}\left(\Sigma_{1,2}\right)$ are defined as follows. We set $q_{i}(\gamma)=i\left(\gamma, \sigma_{i}\right) \geq 0$. Since $\sigma_{1}, \sigma_{2}$ together bound a pair of pants we note

$$
q_{1}+q_{2} \cong 0 \bmod 2 .
$$

This equation will be important in Section 7.

The definition of $p_{i}(\gamma)$ (which is more complicated and can be omitted at first reading) is made relative to the fundamental domain $\Delta$ of Section 2.2. The reader may find the discussion for $\Sigma_{1,1}$ in Appendix 2 enlightening. 


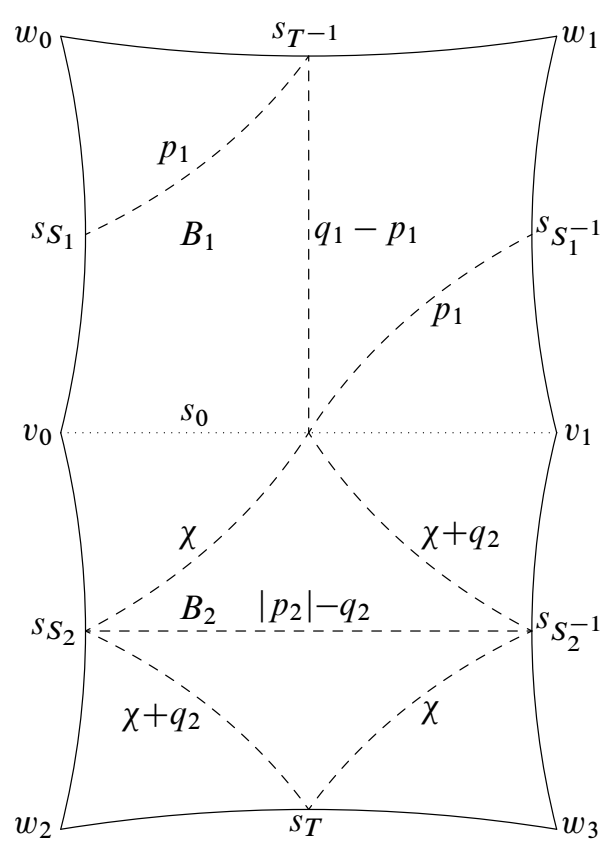

Figure 4: Typical configuration for canonical coordinates. The cell shown is defined by the five inequalities $p_{1} \geq 0, p_{2} \leq 0, q_{1} \geq p_{1}, q_{2} \leq-p_{2}, q_{2} \leq q_{1}$.

Referring to Figure 4, label each side of $\Delta$ by the generator which carries it to a paired side, so that the bottom side is labelled $s_{T}$ since $T$ carries it to the top side $s_{T^{-1}}$; similarly the top left side is labelled $s_{S_{1}}$ since $S_{1}$ carries it to the right side $s_{S_{1}^{-1}}$; and the lower left and right sides are labelled $s_{S_{2}}, s_{S_{2}^{-1}}$ respectively. Since each side joins a puncture to a puncture, the intersection numbers of a curve $\gamma \in \mathcal{S}$ with these sides are well defined. Let $s_{0}$ be the arc joining the vertices $v_{0}, v_{1}$ in the middle of the vertical sides of $\Delta$, which both project to the puncture $S_{2} S_{1}^{-1}$ on $\Sigma_{1,2}$. This partitions $\Delta$ into two four sided "boxes" $B_{1}, B_{2}$ with sides $s_{0}, s_{S_{i}}, s_{S_{i}}{ }^{-1}$ and $s_{T^{-1}}$ or $s_{T}$ respectively.

The geodesic representative of $\gamma$ on $\Delta$ intersects each box $B_{i}$ in a number of pairwise disjoint arcs, none of which runs from one side of $B_{i}$ to itself. We observe [14, Lemma 4.1] that in at least one box $B_{i}$, there is either no "corner arc" joining $s_{0}$ to $s_{S_{i}}$, or no corner arc joining $s_{0}$ to $s_{S_{i}}{ }^{-1}$. This is because if strands of $\gamma$ included all four corner arcs in both boxes, the "innermost" such arcs would link to form a loop round the puncture $S_{2} S_{1}^{-1}$ which is the projection to $\Sigma_{1,2}$ of $v_{0}$ and $v_{1}$. This is impossible. In a similar way, there cannot be corner arcs surrounding all of the four vertices $w_{0}, \ldots, w_{3}$ marked in Figure 4, since their innermost strands would link to form a loop round the common projection of the $w_{i}$, the puncture $S_{1} T S_{2}^{-1} T^{-1}$. Using this together with the "switch 
conditions" $i\left(\gamma, s_{S_{i}}\right)=i\left(\gamma, s_{S_{i}^{-1}}\right)$ and $i\left(\gamma, s_{T}\right)=i\left(\gamma, s_{T^{-1}}\right)$, one checks (see Keen, Parker and Series [14, Section 4]) that also $i\left(s_{0}, \gamma\right)=i\left(s_{T}, \gamma\right)$. It also follows that there are equal number of arcs joining each pair of diagonally opposite corners of each $B_{i}$.

Suppose for definiteness that, as illustrated in Figure 4, the box with missing corner arcs is $B_{1}$. In this situation, it is not hard to see that $q_{1}(\gamma)=i\left(\gamma, \sigma_{1}\right)=i\left(\gamma, s_{0}\right)$. In analogy with the case of $\Sigma_{1,1}$ as described in Appendix 2, we define $\left|p_{1}\right|=i\left(\gamma, s_{S_{1}}\right)=$ $i\left(\gamma, s_{S_{1}^{-1}}\right)$. Since $i\left(\gamma, s_{0}\right) \geq i\left(\gamma, \sigma_{2}\right)=q_{2}(\gamma)$, we have $q_{1} \geq q_{2}$. Set $\chi=\left|q_{2}-q_{1}\right| / 2$. (Note that $\chi$ is integral by (1).) One verifies that $q_{2}(\gamma)=i\left(\gamma, s_{0}\right)-2 \chi \geq 0$ and we define $\left|p_{2}\right|=i\left(\gamma, s_{S_{2}}\right)-2 \chi=i\left(\gamma, s_{S_{2}^{-1}}\right)-2 \chi$.

To fix the sign of $p_{1}$, take $p_{1}>0$ if there is an arc of $\gamma$ joining $s_{S_{1}}$ to $s_{T^{-1}}$ and $p_{1} \leq 0$ otherwise. Likewise take $p_{2}>0$ if there are $w>\chi$ arcs of $\gamma$ joining $s_{S_{2}}$ to $s_{0}$ and $p_{2} \leq 0$ otherwise.

Finally, if $B_{2}$ is the box with missing corner arcs, we have $q_{2} \geq q_{1}$ and we make similar definitions interchanging the indices 1,2 .

The intuition behind this definition is that we are thinking of each $B_{i}$ as a fundamental domain for a once punctured torus $\Sigma_{1,1}^{i}$ with opposite sides identified. With such an identification, the arcs of $\gamma$ in $B_{i}$ would glue up to form a multiple loop on $\Sigma_{1,1}^{i}$. As is well known, closed curves on $\Sigma_{1,1}$ are in bijective correspondence with lines of rational slope in $\mathbb{R}^{2}$. The coordinate $\left(q_{i}, p_{i}\right)$ indicates that the "slope" of $\gamma$ in $B_{i}$ is $q_{i} / p_{i}$; see Appendix 2. The number $\chi$ is the number of "corner strands" joining adjacent sides of $B_{i}$ which, were the box actually $\Sigma_{1,1}$, would link to form $\chi$ copies of a loop round the puncture. Such corner strands occur in box $B_{2}$ if and only if $q_{1}>q_{2}$; this is why we subtracted $\chi$ from the $B_{2}$-intersection numbers only. Note that, in contrast to $\Sigma_{1,1}$, the numbers $q_{i}, p_{i}$ are no longer in general relatively prime. Up to the choice of a base point for the twist, $p_{i} / q_{i}$ is the Dehn-Thurston twist coordinate of $\gamma$ relative to $\sigma_{i}$; see either Maloni and Series [18] or Minsky [23, Lemma 3.5] and Section 6.4 below.

The connection between this definition and the more standard cell decomposition of $M L$ by weighted train tracks is as follows. Collapse all the arcs of $\gamma$ joining one side of $B_{i}$ to another, into a single strand joining the midpoints of the same two sides. The collapsed strands join across $s_{0}$ to form a train track on $\Sigma_{1,2}$, whose branches are the strands and all of whose switches are at the midpoints of the sides (including $s_{0}$ ). In [14] we called these special tracks, $\pi_{1,2}$-train tracks; here we refer to them as canonical. The nonnegative weights on this collection of train tracks form a cell decomposition of $M L\left(\Sigma_{1,2}\right)$. However the coordinates $\left(q_{i},\left|p_{i}\right|\right)$ are not in general equal to the actual weights on these rather complicated configurations of branches. Rather, $\mathbf{i}(\gamma)$ has global 
meaning. Inequalities among the coefficients of $\mathbf{i}(\gamma)$ determine which track in the cell decomposition supports a given curve $\gamma$. Precisely, there are 32 cells determined by the inequalities

$$
q_{1} \geq q_{2}, q_{1} \leq q_{2} \quad \text { and } \quad p_{i} \geq 0, p_{i} \leq 0 ; \quad\left|p_{i}\right| \geq q_{i},\left|p_{i}\right| \leq q_{i} \quad \text { for } i=1,2 .
$$

Given the cell, the coefficients determine the weights on the track.

Canonical coordinates extend naturally by linearity and continuity to global coordinates for $M L\left(\Sigma_{1,2}\right)$. The cell structure is best understood by referring to Figure 9 in Appendix 2. This shows the four cells for $\Sigma_{1,1}$ which glue to form $\operatorname{PML}\left(\Sigma_{1,1}\right)=S^{1}$. Each of the four configurations in Figure 9 can occur in either $B_{1}$ or $B_{2}$, and for each such configuration there are 2 further options for the box in which $q_{i}>q_{j}$, This makes in all 32 cells, the images of which in $P M L$ glue along their faces to make $S^{3}$ [14].

An important feature of canonical coordinates is that it is easy to read off the coordinates of a curve $\gamma_{W} \in \mathcal{S}$ represented by a word $W$ in the generators $S_{1}, S_{2}, T$ of $\pi_{1}(\Sigma)$. First, write $W$ as a cyclically shortest word $e_{1} e_{2} \cdots e_{n}$ and set $e_{n+1}=e_{1}$. Draw arcs on $\Delta$ from $s_{e_{i}}$ to $s_{e_{i+1}^{-1}}, i=1, \ldots, n$. Suppose that $\gamma_{W}$ is simple on $\Sigma$. Then by [14, Theorem 3.1], these arcs can be arranged so as to be pairwise disjoint and the weighted canonical track they define gives precisely the canonical coordinates of $\gamma_{W}$. This method, similar to the method of $\pi_{1}$-train tracks developed at length in [4], was crucial in the proof of the top terms formula below. Some examples are given in Section 5.

\subsection{Top terms formula}

Canonical train tracks and coordinates were used in [14] to study matrices $\rho(W)$ in the family $\mathcal{G}$ of Section 2.2 , where $W \in \pi_{1}\left(\Sigma_{1,2}\right)$ corresponds to $\gamma_{W} \in \mathcal{S}$. The matrix coefficients and hence the trace $\operatorname{Tr} W$ are clearly polynomials in $\tau_{1}, \tau_{2}$.

Theorem 4.1 [14, Theorem 6.1] Let $\gamma$ be a simple closed curve on the twice punctured torus $\Sigma_{1,2}$ with canonical coordinates $\mathbf{i}(\gamma)=\left(q_{1}, p_{1}, q_{2}, p_{2}\right)$. Let $\gamma$ be represented by $W \in \pi_{1}(\Sigma)$. Then if $q_{1}, q_{2}>0$

$$
\operatorname{Tr} W= \pm 2^{\left|q_{2}-q_{1}\right|}\left(\tau_{1}+2 p_{1} / q_{1}\right)^{q_{1}}\left(\tau_{2}+2 p_{2} / q_{2}\right)^{q_{2}}+R\left(q_{1}+q_{2}-2\right)
$$

where $R\left(q_{1}+q_{2}-2\right)$ denotes a polynomial of degree at most $q_{1}$ in $\tau_{1}$ and $q_{2}$ in $\tau_{2}$ and with total degree in $\tau_{1}$ and $\tau_{2}$ at most $q_{1}+q_{2}-2$. If $q_{2}=0$ then $\operatorname{Tr} W$ is a polynomial in $\tau_{1}$ only, and

$$
\operatorname{Tr} W= \pm 2^{q_{1}}\left(\tau_{1}+2 p_{1} / q_{1}\right)^{q_{1}}+R\left(q_{1}-2\right),
$$

while if $q_{1}=0$ there is a similar expression in $\tau_{2}$. 
Remark 4.2 An analogous formula for an arbitrary hyperbolisable surface is the main result of [18].

\subsection{The Thurston symplectic form}

Thurston defined a symplectic form $\Omega_{\mathrm{Th}}$ on $M L$, the symplectic product being defined for curves carried by a common train track $\tau$; see for example Penner and Harer [26]. By splitting, we can arrange that every switch $v$ of $\tau$ is trivalent with one incoming branch and two outgoing ones. Since $\Sigma$ is oriented, we can distinguish the right and left hand outgoing branches, the left hand branch being the one to be followed by a British driver approaching $v$ from the incoming branch; see Figure 5. If $\mathbf{n}, \mathbf{n}^{\prime}$ are nonnegative weightings on $\tau$ (representing points in $M L$ ), we denote by $b_{v}(\mathbf{n}), c_{v}(\mathbf{n})$ the weights of the left hand and right hand outgoing branches at $v$ respectively. The Thurston product is defined as $\Omega_{\mathrm{Th}}\left(\mathbf{n}, \mathbf{n}^{\prime}\right)=\frac{1}{2} \sum_{v} b_{v}(\mathbf{n}) c_{v}\left(\mathbf{n}^{\prime}\right)-b_{v}\left(\mathbf{n}^{\prime}\right) c_{v}(\mathbf{n})$.

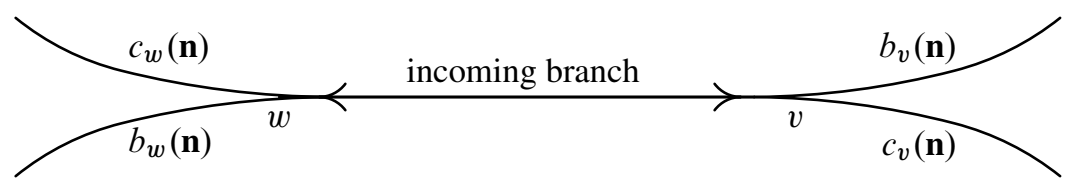

Figure 5: Weighted branches at a switch

Bearing in mind the interpretation of canonical coordinates as weights on train tracks, we can interpret this definition in terms of canonical coordinates. It is then not hard to check the following rather remarkable result:

Proposition 4.3 Suppose that loops $\gamma, \gamma^{\prime} \in \mathcal{S}$ are supported on a common canonical train track with coordinates $\mathbf{i}(\gamma)=\left(q_{1}, p_{1}, q_{2}, p_{2}\right), \mathbf{i}\left(\gamma^{\prime}\right)=\left(q_{1}^{\prime}, p_{1}^{\prime}, q_{2}^{\prime}, p_{2}^{\prime}\right)$. Then $\Omega_{\mathrm{Th}}\left(\gamma, \gamma^{\prime}\right)=\sum_{i=1,2}\left(q_{i}\left|p_{i}^{\prime}\right|-q_{i}^{\prime}\left|p_{i}\right|\right)$. If $\gamma, \gamma^{\prime}$ are disjoint, then $\Omega_{\mathrm{Th}}\left(\gamma, \gamma^{\prime}\right)=0$.

To check the last statement, note first that disjoint curves are necessarily supported on the same canonical track. Then check that $\Omega_{\mathrm{Th}}$ is invariant under splitting and shifting. Then split and shift until the two curves are supported on disjoint train tracks.

\section{Computation and examples}

Before embarking on the proof of Theorem A, we briefly discuss its implications for computation and then give a few examples which it is possible to work out by hand. 


\subsection{Computation}

Let us return to the original problem of locating the Maskit slice $\mathcal{M}$. Conjecturally, the pleating planes are dense in $\mathcal{M}$. (This was proved for $\mathcal{M}\left(\Sigma_{1,1}\right)$ in [15].) Thus we concentrate on the problem of locating a given pleating plane $\mathcal{P}_{\gamma_{1}, \gamma_{2}}$ in the parameter space $\mathbb{H}^{2} \subset \mathbb{C}^{2}$. Let $\mathcal{V}_{>2}\left(\gamma_{i}\right), \mathcal{V}_{=2}\left(\gamma_{i}\right)$ denote respectively the real analytic varieties in $\mathbb{H}^{2}$ on which $\operatorname{Tr} \gamma_{i} \in \mathbb{R} \backslash\{[-2,2]\}$ and $\operatorname{Tr} \gamma_{i}= \pm 2$. By Theorem 3.4, $\mathcal{P}_{\gamma_{1}, \gamma_{2}}$ is a connected totally real submanifold of $\mathcal{V}_{>2}\left(\gamma_{1}\right) \cap \mathcal{V}_{>2}\left(\gamma_{2}\right)$. Its boundary is contained in $\mathcal{V}_{=2}\left(\gamma_{1}\right) \cup \mathcal{V}_{=2}\left(\gamma_{2}\right)$ and the two rays $\mathcal{P}_{\gamma_{1}}$ and $\mathcal{P}_{\gamma_{2}}$. By Theorem $\mathrm{B}$, as long as the pair $\gamma_{1}, \gamma_{2}$ is not exceptional (for which see Lemma 7.4 and Theorem 7.5), $\mathcal{V}_{>2}\left(\gamma_{1}\right) \cap \mathcal{V}_{>2}\left(\gamma_{2}\right)$ is a 2 -manifold and has a unique branch near infinity satisfying the conditions of Theorem A.

The ray $\mathcal{P}_{\gamma_{1}}$ may be determined as follows. At points on $\mathcal{P}_{\gamma_{1}}$, the upper component of the convex hull boundary $\partial \mathcal{C}^{+} / G$ cut along $\gamma_{1}$ is flat. Therefore the trace of any curve $\delta$ disjoint from $\gamma_{1}$ lies in the variety $\mathcal{V}_{>2}(\delta)$. One can show (see Lemma 7.2) that among any two admissible curves $\delta, \delta^{\prime}$ disjoint from $\gamma_{1}$ and distinct from $\gamma_{2}$, at least one will have the property that $\mathcal{V}_{>2}(\delta) \cup \mathcal{V}_{>2}\left(\gamma_{1}\right)$ is transverse to $\mathcal{P}_{\gamma_{1}, \gamma_{2}}$, so that the equations $\Im \gamma_{1}, \Im \delta, \Im \delta^{\prime} \in \mathbb{R}$ together with the conditions of Theorem $\mathrm{A}$ are enough to determine $\mathcal{P}_{\gamma_{1}}$. Finally, since $\mathcal{P}_{\gamma_{1}, \gamma_{2}}$ is the union of the rays $\mathcal{P}_{\xi}$ for $\xi$ with support $\gamma_{1}, \gamma_{2}$, it must be the connected component of the unique branch of $\mathcal{V}_{>2}\left(\gamma_{1}\right) \cap \mathcal{V}_{>2}\left(\gamma_{2}\right)$ which interpolates between $\mathcal{P}_{\gamma_{1}}$ and $\mathcal{P}_{\gamma_{2}}$ as $\tau_{i} \rightarrow \infty$.

This means that in principle, given a means of computing sufficiently many traces, $\mathcal{P}_{\gamma_{1}, \gamma_{2}}$ can be determined computationally without needing any further tests to see if $G$ is discrete. In particular, one can locate the one (real)-dimensional boundary of $\mathcal{P}_{\gamma_{1}, \gamma_{2}}$ on $\partial \mathcal{M}$, along which at least one of the elements $\gamma_{1}, \gamma_{2}$ is parabolic. By [21], such geometrically finite group cusp groups are dense in $\partial \mathcal{M}$. In the one dimensional case, this is exactly the procedure which gives Figure 1. We hope to discuss the practical implementation of this programme elsewhere; see Austin [1] and Austin and Series [2].

\subsection{Examples}

Following [13], we will identify pleating rays and planes formed by various combinations of the curves corresponding to the elements $W=S_{i}, T,\left[S_{i}, T^{-1}\right], S_{1} S_{2}^{-1} T^{-1}$ and $S_{1}^{-1} S_{2} T^{-1}$ in $\pi_{1}(\Sigma)$. Let $\gamma_{W}$ be the curve corresponding to the element $W$ and write $\mathcal{P}_{W}$ for $\mathcal{P}_{\gamma_{W}}$ and $\operatorname{Tr} W$ for $\operatorname{Tr} \rho(W)$ with $\rho=\rho\left(\tau_{1}, \tau_{2}\right)$ defined as in Section 2.2.

We compute $\operatorname{Tr} T=2+\tau_{1} \tau_{2}, \operatorname{Tr}\left[S_{i}, T^{-1}\right]=2+4 \tau_{j}^{2}, j \neq i$ (see Appendix 1), and $\operatorname{Tr} S_{1} S_{2}^{-1} T^{-1}=\left(\tau_{2}-2\right)\left(\tau_{1}+2\right)$, where $\operatorname{Tr} S_{1} S_{2}^{-1} T^{-1}$ can either be computed 
directly or deduced from the fact that the right Dehn twist $D_{\sigma_{i}}$ about $S_{i}$ induces the map $\tau_{i} \mapsto \tau_{i}+2$; see Section 2.2. Writing the canonical coordinates in the usual form

$$
\mathbf{i}(\xi)=\left(q_{1}(\xi), p_{1}(\xi), q_{2}(\xi), p_{2}(\xi)\right),
$$

we find following the method outlined in Section 4:

$$
\begin{gathered}
\mathbf{i}(T)=(1,0,1,0), \quad \mathbf{i}\left(\left[S_{1}, T^{-1}\right]\right)=(0,0,2,0), \quad \mathbf{i}\left(\left[S_{2}, T^{-1}\right]\right)=(2,0,0,0), \\
\mathbf{i}\left(S_{1} S_{2}^{-1} T^{-1}\right)=(1,-1,1,1), \quad \mathbf{i}\left(S_{1}^{-1} S_{2} T^{-1}\right)=(1,1,1,-1) .
\end{gathered}
$$

The curves $S_{1} S_{2}^{-1} T^{-1}$ and $S_{1} T^{-1} S_{1}^{-1} T$ are illustrated in Figure 6.
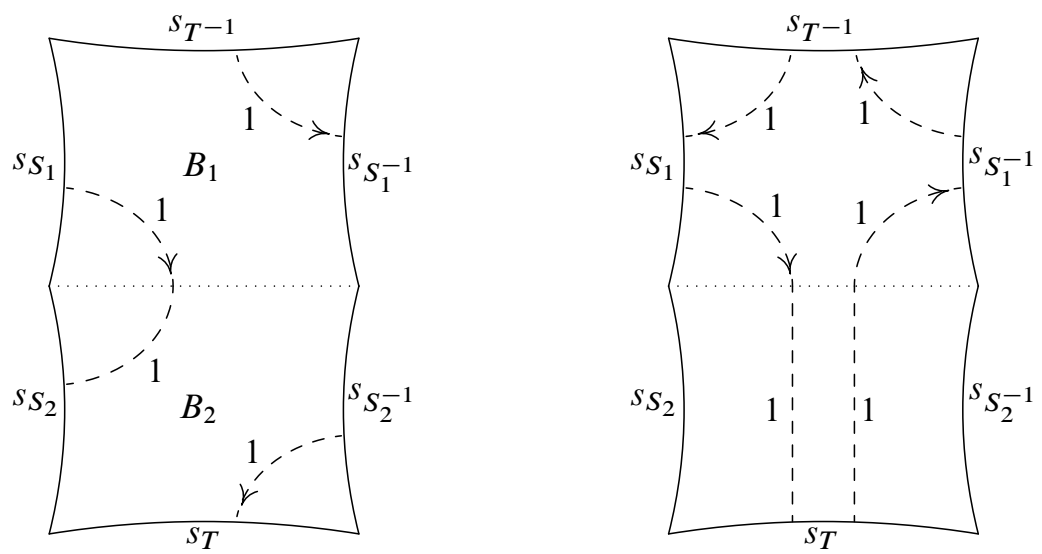

Figure 6: The curves $S_{1} S_{2}^{-1} T^{-1}$ (left) and $S_{1} T^{-1} S_{1}^{-1} T$ (right)

The pleating planes discussed in Examples 1-3a below are shown in the left frame of Figure 7 and those of Example 4 on the right.

Example 1 (The pleating ray $\mathcal{P}_{T}$ ) On $\mathcal{P}_{T}$, the surface $\Sigma_{T}:=\Sigma \backslash \gamma_{T}$ is flat and hence not only $\gamma_{T}$, but also any curve contained in $\Sigma_{T}$, has real trace. Since $\gamma_{T}$ is disjoint from both $\gamma_{\left[S_{1}, T^{-1}\right]}$ and $\gamma_{\left[S_{2}, T^{-1}\right]}$, it follows that $2+4 \tau_{j}^{2} \in \mathbb{R}, i=1,2$ and $2+\tau_{1} \tau_{2} \in \mathbb{R}$. We deduce that on $\mathcal{P}_{T}, \mathfrak{R} \tau_{1}=\Re \tau_{2}=0$. Since $\gamma_{T}$ is also disjoint from $\gamma_{S_{1} S_{2}^{-1} T^{-1}}$, by the same reasoning, $\tau_{1} \tau_{2}+2 \tau_{2}-2 \tau_{1} \in \mathbb{R}$, from which we deduce $\Im \tau_{1}=\Im \tau_{2}$. (Disjointness of curves can be easily checked by drawing disjoint representatives on the fundamental domain $\Delta$, taking into account how the arcs link across the glued sides. In this instance, the curve $T$ is an arc from $s_{T}$ to $s_{T^{-1}}$ which is clearly disjoint from both curves illustrated in Figure 6.) 

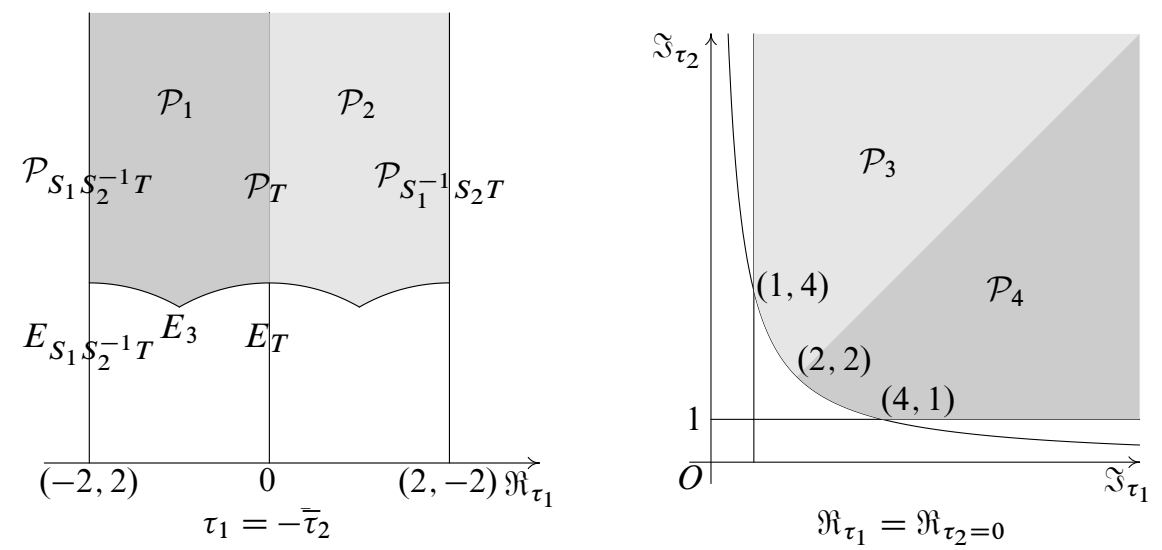

Figure 7: Pleating planes. Left: Examples 1-3a. Right: Example 4.

The conditions $\Re \tau_{1}=\Re \tau_{2}=0$, $\Im \tau_{1}=\mathfrak{\Im} \tau_{2}$ define a line $L$ in $\mathbb{C}_{+}^{2}$. Moreover $|\operatorname{Tr} T|>2$ on $L$ whenever $\Im \tau_{1}>2$, and $T$ is parabolic exactly at the point at which $\Im \tau_{1}=2$. It follows from Theorem 3.4 and the discussion above, that $\mathcal{P}_{T}=L \cap\left\{\mathfrak{s} \tau_{1}>2\right\}$. To compare with Theorem A, since $p_{i}(T) / q_{i}(T)=0, i=1,2$ and $q_{2}(T) / q_{1}(T)=1$, in this special case, $\mathcal{P}_{T}$ is actually equal to the line

$$
\Re \tau_{i}=2 p_{i}(T) / q_{i}(T), \operatorname{Arg} \tau_{i}=\pi / 2, \Im \tau_{1} / \Im \tau_{2}=q_{2}(T) / q_{1}(T) .
$$

The endpoint point $E_{T}=(2 i, 2 i)$ of $\mathcal{P}_{T}$ represents the unique group $G_{\text {cusp }}(T)$ for which $S_{1}, S_{2}$ and $T$ are all parabolic and the remaining part of $\partial \mathcal{C}^{+} / G$ is totally geodesic.

In this very special case, it is also possible to verify directly, following the methods of [15], that groups on $L$ have convex hull boundary which is bent exactly along the curve $\gamma_{T}$, and that by symmetry the geodesic axis of $\gamma_{T}$ meets both the geodesic representatives of $\sigma_{i}=\gamma_{S_{i}}$ on $\partial \mathcal{C}^{+} / G$ orthogonally.

Example 2 (The pleating ray $\mathcal{P}_{S_{1} S_{2}^{-1} T^{-1}}$ ) The easy way to locate this ray is to note that $S_{1} S_{2}^{-1} T^{-1}=D_{\sigma_{1}} D_{\sigma_{2}}^{-1}(T)^{2}$. Since $D_{\sigma_{i}}$ is a symmetry of $\mathcal{M}$, it follows immediately that $\mathcal{P}_{S_{1} S_{2}^{-1} T^{-1}}$ is the line $\Re \tau_{1}=-2, \Re \tau_{2}=2$, $\Im \tau_{1}=\Im \tau_{2}, \Im \tau_{1}>2$, in other words, the line $L+2(-1,1)$ where $L$ is as in Example 1. Since in this case $p_{1} / q_{1}=-1, p_{2} / q_{2}=1$ and $q_{2} / q_{1}=1$, this is again in accordance with Theorem A.

The point $E_{S_{1} S_{2}^{-1} T^{-1}}=(-2+2 i, 2+2 i)$ is the unique group for which $S_{1}, S_{2}$ and $S_{1} S_{2}^{-1} T^{-1}$ are all parabolic and the remaining part of $\partial \mathcal{C}^{+} / G$ is totally geodesic. 


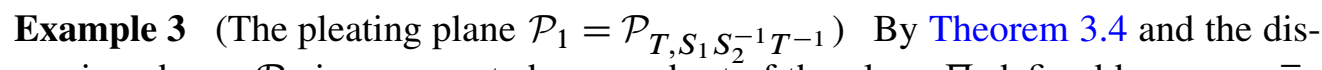
cussion above, $\mathcal{P}_{1}$ is a connected open subset of the plane $\Pi$ defined by $\tau_{1}=-\bar{\tau}_{2}$ whose boundary contains the lines $\mathcal{P}_{T}$ and $\mathcal{P}_{S_{1} S_{2}^{-1} T^{-1}}$. To compute the remaining boundary of $\mathcal{P}_{1}$, note that the conditions $|\operatorname{Tr} T|,\left|\operatorname{Tr} S_{1} S_{2}^{-1} T^{-1}\right|>2$ imply $\left|\tau_{1}\right|^{2}>4,\left|\tau_{1}-2\right|^{2}>4$. The equations $\left|\tau_{1}\right|=2,\left|\tau_{1}-2\right|=2$ define two circular arcs which meet at the point $E_{3}$ at which $\Re \tau_{1}=-1, \Im \tau_{1}=\sqrt{3}$. Then $T$ is parabolic along the arc of $\left|\tau_{1}\right|=2$ from $E_{T}$ to $E_{3}$ and $S_{1} S_{2}^{-1} T^{-1}$ is parabolic along the arc of $\left|\tau_{1}-2\right|=2$ from $E_{S_{1} S_{2}^{-1} T^{-1}}$ to $E_{3}$. At $E_{3}$, both $T$ and $S_{1} S_{2}^{-1} T^{-1}$ are parabolic. Thus $E_{3}$ represents a double cusp group in which $T, S_{1} S_{2}^{-1} T^{-1}, S_{1}, S_{2}$ are all parabolic. (There is a unique such group, either by the ending lamination theorem, or more simply by [12].) It follows from the above discussion that

$$
\mathcal{P}_{T, S_{1} S_{2}^{-1} T^{-1}}=\left\{\left(\tau_{1}, \tau_{2}\right)\left|\tau_{1}=-\bar{\tau}_{2},\right| \tau_{1}|>2,| \tau_{1}-2 \mid>2,-2<\Re \tau_{1}<0\right\},
$$

as illustrated in the left frame of Figure 7.

The next example shows that distinct planes may be contained in the same real trace variety in $\mathbb{C}_{+}^{2}$.

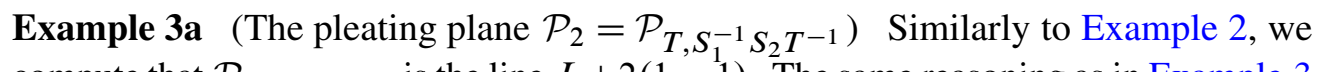
compute that $\mathcal{P}_{S_{1}^{-1} S_{2} T^{-1}}$ is the line $L+2(1,-1)$. The same reasoning as in Example 3 shows that $\mathcal{P}_{T, S_{1}^{-1} S_{2} T^{-1}}$ is the region contained the same plane $\Pi$, but bounded by the lines $\mathcal{P}_{T}$ and $\mathcal{P}_{S_{1}^{-1}}^{-1} S_{2} T^{-1}$ and above the arcs $\left|\tau_{1}\right|=2$ and $\left|\tau_{1}+2\right|=2$. In other words

$$
\mathcal{P}_{T, S_{1}^{-1} S_{2} T^{-1}}=\left\{\left(\tau_{1}, \tau_{2}\right)\left|\tau_{1}=-\bar{\tau}_{2},\right| \tau_{1}|>2,| \tau_{1}+2 \mid>2,0<\Re \tau_{1}<2\right\} .
$$

Thus the two pleating planes $\mathcal{P}_{1}$ and $\mathcal{P}_{2}$ are both contained same plane $\Pi \subset \mathbb{C}_{+}^{2}$. They meet along the line $\mathcal{P}_{T}$ contained in the boundary of both; see the left frame of Figure 7.

Our final example is of degenerate pleating planes.

Example 4 (The pleating planes $\mathcal{P}_{T,\left[S_{i}, T^{-1}\right]}$ ) In Example 3, the curves $T$ and $S_{1} S_{2}^{-1} T^{-1}$ are themselves are admissible, so that the corresponding pleating rays are nonempty. By contrast, since $q_{1}=i\left(\left[S_{1}, T^{-1}\right], S_{1}\right)=0$, the curve $\left[S_{1}, T^{-1}\right]$ by itself is not admissible so that $\mathcal{P}_{\left[S_{1}, T^{-1}\right]}=\varnothing$.

From $\operatorname{Tr} T=2+\tau_{1} \tau_{2}, \operatorname{Tr}\left[S_{1}, T^{-1}\right]=2+4 \tau_{2}^{2}$ we find $\mathcal{P}_{T,\left[S_{i}, T^{-1}\right]}$ is contained in the plane $\Pi^{\prime}=\left\{\left(\tau_{1}, \tau_{2}\right) \in \mathbb{C}_{+}^{2}: \Re \tau_{1}=\Re \tau_{2}=0\right\}$. To locate $\mathcal{P}_{T,\left[S_{i}, T^{-1}\right]}$, we locate its boundary curves in $\Pi^{\prime}$. Part of the boundary is the line $\mathcal{P}_{T}=L$ of Example 1, along which $\Im \tau_{1}=\Im \tau_{2}$. In $\Pi^{\prime}$, the element $\left[S_{1}, T^{-1}\right]$ is parabolic along the line $\tau_{2}=i$ and $T$ is parabolic along the hyperbola $\left\{\mathfrak{\Im} \tau_{1} \Im \tau_{2}=4\right\}$. These two loci meet exactly once at the point $(4 i, i)$ which therefore represents the maximally pinched group for 
which $S_{1}, S_{2}, T$ and $\left[S_{i}, T^{-1}\right]$ are all parabolic. We conclude that $\mathcal{P}_{T,\left[S_{1}, T^{-1}\right]}$ is the region $\mathcal{P}_{4}$ in Figure 7 bounded by the line $\{(t i, t i): t \geq 2\}$, the $\operatorname{arc} A_{1}$ of $\left\{\Im \tau_{1} \Im \tau_{2}=4\right\}$ from $(2 i, 2 i)$ to $(4 i, i)$ along which $T$ is parabolic; and the line $A_{2}=\{(t i, i): t \geq 4\}$ along which $\left[S_{1}, T\right]$ is parabolic. Along $A_{1}$, the bending angle $\theta_{\left[S_{1}, T^{-1}\right]}$ increases from zero to $\pi$ while $\theta_{T} \equiv \pi$; along $A_{2}$, we have $\theta_{\left[S_{1}, T^{-1}\right]} \equiv \pi$ while $\theta_{T}$ decreases from $\pi$ to the unattainable value 0 .

The individual pleating ray $\mathcal{P}_{\xi}$ with $\xi=a_{1} T+a_{2}\left[S_{i}, T\right]$, is asymptotic to the line $\Im \tau_{2} / \Im \tau_{1}=q_{1}(\xi) / q_{2}(\xi)=a_{1} /\left(a_{1}+a_{2}\right)$; the missing fourth boundary curve of $\mathcal{P}_{T,\left[S_{1}, T^{-1}\right]}$ would correspond to the nonexistent ray $\mathcal{P}_{\left[S_{1}, T^{-1}\right]}$ asymptotic to the line $\Im \tau_{2} / \Im \tau_{1} \rightarrow q_{1}\left(\left[S_{1}, T^{-1}\right]\right) / q_{2}\left(\left[S_{1}, T^{-1}\right]\right)=0$.

A similar argument shows that

$$
\overline{\mathcal{P}_{T,\left[S_{2}, T^{-1}\right]}}=\left\{\left(\tau_{1}, \tau_{2}\right) \in \Pi^{\prime}: 1 \leq \Im \tau_{1} \leq \Im \tau_{2}, \Im \tau_{1} \Im \tau_{2} \geq 4\right\},
$$

the region $\mathcal{P}_{3}$ in the figure. Thus $\mathcal{P}_{3}$ and $\mathcal{P}_{4}$ are contained in the same plane in $\mathbb{C}_{+}^{2}$. Also note that the curves

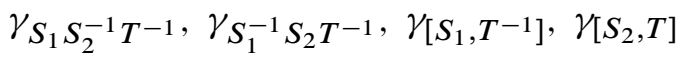

are all disjoint from $\gamma_{T}$ and that the ray $\mathcal{P}_{T}$ is the common boundary of all four pleating planes $\mathcal{P}_{1}, \ldots, \mathcal{P}_{4}$.

\section{Behaviour on a pleating variety}

The heart of the proof of Theorem A is the geometry of the upper component $\partial \mathcal{C}^{+} / G$ of the convex hull boundary for groups $G=G_{\xi}(\theta)$ along the pleating ray $\mathcal{P}_{\xi}$ as $\theta \rightarrow 0$. Let $\sigma^{+}=\sigma_{i}^{+}$denote the geodesic representative of $\sigma_{i}$ on $\partial \mathcal{C}^{+} / G$ and let $l_{\sigma}^{+}=l_{\sigma_{i}}^{+}$ be its hyperbolic length in the hyperbolic structure on $\partial \mathcal{C}^{+} / G$. We show that $l_{\sigma}^{+} \rightarrow 0$ as $\theta \rightarrow 0$, while $\sigma^{+}$becomes asymptotically orthogonal to the bending lines. From this we deduce results on the asymptotic behaviour of $\tau_{1}, \tau_{2}$. The main result of this section will be:

Theorem 6.1 Fix an admissible rational measured lamination $\xi=\sum_{1,2} a_{i} \delta_{\gamma_{i}}$ on the twice punctured torus $\Sigma_{1,2}$ and let $G=G_{\xi}(\theta)$ be the unique group in the Maskit slice $\mathcal{M}$ with bending measure $\beta(G)=\theta \xi$. Then the coordinates $\left(\tau_{1}, \tau_{2}\right) \in \mathcal{M} \subset \mathbb{C}^{2}$ satisfy

$$
\Re \tau_{i}=-2 p_{i}(\xi) / q_{i}(\xi)+O(1) \quad \text { and } \quad \Im \tau_{i}=4(1+O(c \theta)) / \theta q_{i}(\xi)
$$

where $\left(p_{i}(\xi), q_{i}(\xi)\right)$ are the canonical coordinates of $\xi, c$ is a constant depending on $q_{1}(\xi), q_{2}(\xi)$, and $O(1)$ denotes a universal bound independent of $\xi$, as $\theta \rightarrow 0$. 


\section{Corollary 6.2 Then}

$$
\left|\arg \tau_{i}-\pi / 2\right| \leq c^{\prime} \theta \quad \text { and } \quad\left|\frac{q_{2}(\xi)}{q_{1}(\xi)}-\frac{\Im \tau_{1}}{\Im \tau_{2}}\right| \leq c^{\prime \prime} \theta
$$

where $c^{\prime}, c^{\prime \prime}>0$ are constants depending on $\xi$, as $\theta \rightarrow 0$.

Theorem 6.1 and the Corollary follow immediately from Propositions 6.6, 6.11 and 6.14. At the end of the section, we also prove Theorem C.

\subsection{A note on constants}

Throughout this section, we will make many estimates of the form $X\left(\sigma_{i}\right) \leq O\left(\theta^{e}\right)$, where $X$ is some quantity which depends on the curve $\sigma_{i}$, meaning that $X \leq c \theta^{e}$ as $\theta \rightarrow 0$ for some constant $c>0$, and $e$ is an exponent (usually $e=1,2$ or $1 / 2$ ). These estimates all depend on the lamination $\xi$, so more precisely one has $X \leq c(\xi) \theta^{e}$. However it is easily seen by following through the arguments that the dependence on $\xi$ is always of the form $X\left(\sigma_{i}\right) \leq c q_{i}(\xi)^{e} \theta^{e}$, where now $c$ is a universal constant independent of $\xi$. The dependence of the constants on $\xi$ is not important for our final arguments in Section 7, but we note it as it may be useful elsewhere.

In what follows, $X \geq-O(\theta) Y$ means that $X \geq-c Y \theta$ for some constant $c>0$.

\subsection{Length estimates on $\partial \mathcal{C}^{+}$}

We begin by estimating the lengths $l_{\sigma_{i}}^{+}$of the geodesic representatives $\sigma_{i}^{+}$of the accidental parabolics $\sigma_{i}$ on the upper convex hull boundary $\partial \mathcal{C}^{+} / G$. We prove two main results, Propositions 6.4 and 6.6, which relate $l_{\sigma_{i}}^{+}$to the bending angle $\theta$ and the complex parameter $\tau$ respectively. The first shows in particular that $l_{\sigma_{i}}^{+} \rightarrow 0$ as $\theta \rightarrow 0$.

We shall several times use the following estimate which is [28, Lemma 5.4]; see also Theorem 4.2 .10 of [6]. Since the proof is so simple, we repeat it here.

Lemma 6.3 Let $\lambda$ be a piecewise geodesic arc in $\mathbb{H}^{3}$ with endpoints $P$ and $P^{\prime}$, and let $\hat{\lambda}$ be the $\mathbb{H}^{3}$ geodesic joining $P$ to $P^{\prime}$. Suppose that for all $X \in \lambda$ the angle between $P X$ and $\lambda$ is bounded in modulus by $a \in(0, \pi / 4)$. Then $l_{\lambda} \geq(\cos a) l_{\lambda}$ for all $X \in \lambda$, where $l_{\lambda}$ and $l_{\hat{\lambda}}$ are the lengths of $\lambda$ and $\hat{\lambda}$ respectively.

Proof Join $P$ to a variable point $X$ on $\lambda$ between $P$ and $P^{\prime}$ (see Figure 3 in [28]). If $P X$ has length $x$, the distance from $P$ to $X$ along $\lambda$ is $t$, and the acute angle between $P X$ and $\lambda$ at $X$ is $\psi$, then at every non-bend point of $\lambda$, one has the usual variational formula $d x / d t=\cos \psi$ (see Lemma 4.2.12 of [6]). 
Proposition 6.4 Suppose that the rational measured lamination $\xi \in M L_{\mathbb{Q}}$ is admissible. As usual, let $G_{\xi}(\theta)$ be the unique group in the Maskit slice $\mathcal{M}$ with bending measure $\beta(G)=\theta \xi$, so that $G$ lies on the pleating ray $\mathcal{P}_{\xi}$. Then

$$
l_{\sigma_{i}}^{+} \leq i\left(\beta\left(G_{\xi}(\theta)\right), \sigma_{i}\right)\left(1+O\left(\theta^{2}\right)\right)=\theta i\left(\xi, \sigma_{i}\right)\left(1+O\left(\theta^{2}\right)\right)
$$

as $\theta \rightarrow 0$.

Proof Here and in what follows, we work in the upper half space model of $\mathbb{H}^{3}$ and let $\partial \mathcal{C}^{+}$denote the lift of $\partial \mathcal{C}^{+} / G$ to $\mathbb{H}^{3}$. Using Lemma 2.3 if needed, normalise such that $S_{i}=S: z \mapsto z+2$. Let $\widetilde{S}^{+}=\widetilde{S}_{i}^{+}$denote the lift of $\sigma_{i}^{+}$to $\partial \mathcal{C}^{+}$invariant under translation $S$. Then $\widetilde{S}^{+}$is made up of a number of $\mathbb{H}^{3}$-geodesic segments which meet at bending points where $\widetilde{S}^{+}$crosses a bending line of $\partial \mathcal{C}^{+}$. Choose a bending point $P=P_{0}$, let $P_{0}, P_{1}, \ldots, P_{k}, P_{k+1}=P^{\prime}$ be in order the bending points along one period of $\tilde{S}^{+}$between $P_{0}$ and $P_{k+1}=S\left(P_{0}\right)$, and let $s_{i}$ be the segment from $P_{i-1}$ to $P_{i}$; see Figure 8 .

Let $\phi_{i}$ denote the exterior angle between the geodesic segments $s_{i}, s_{i+1}$ of $\widetilde{S}^{+}$which meet at $P_{i}$, measured so that $\phi_{i} \geq 0$ and so that $\phi_{i}=0$ means that $s_{i}, s_{i+1}$ are collinear. (This will be the case exactly when the bending line containing $P_{i}$ is contained in the interior of a flat piece of $\partial \mathcal{C}^{+}$.) If $\theta_{i}$ is the bending angle of $\partial \mathcal{C}^{+}$at $P_{i}$, then $\phi_{i} \leq \theta_{i}$; see Appendix 3. Hence

$$
\sum_{i=0}^{k} \phi_{i} \leq i\left(\sigma, \beta\left(G_{\xi}(\theta)\right)=\theta i(\sigma, \xi)\right.
$$

Now consider the triangle $\Delta$ with vertices $\infty, P, P^{\prime}$. Since $P, P^{\prime}$ are at the same Euclidean height above $\mathbb{C}$ in $\mathbb{H}^{3}$, the angles in $\Delta$ at $P$ and $P^{\prime}$ are equal, to $w / 2$ say. Let $E$ be the (nonplanar) polygon bounded by the $\mathbb{H}^{3}$-geodesic $P P^{\prime}$ and the segments $s_{i}$ of $\tilde{S}^{+}$, and let $u, v$ be the interior angles in $E$ at $P, P^{\prime}$ respectively; see Figure 8. The Euclidean translation $S$ carries the Euclidean configuration at $P$ to that at $P^{\prime}=S(P)$. It follows (using the triangle inequality in the spherical metric on the link of $P$ ) that

$$
\phi_{0}+u+v+w \geq \pi
$$

Summing over the interior angles of $E$ gives

$$
\sum_{1}^{k}\left(\pi-\phi_{i}\right)+u+v<k \pi .
$$




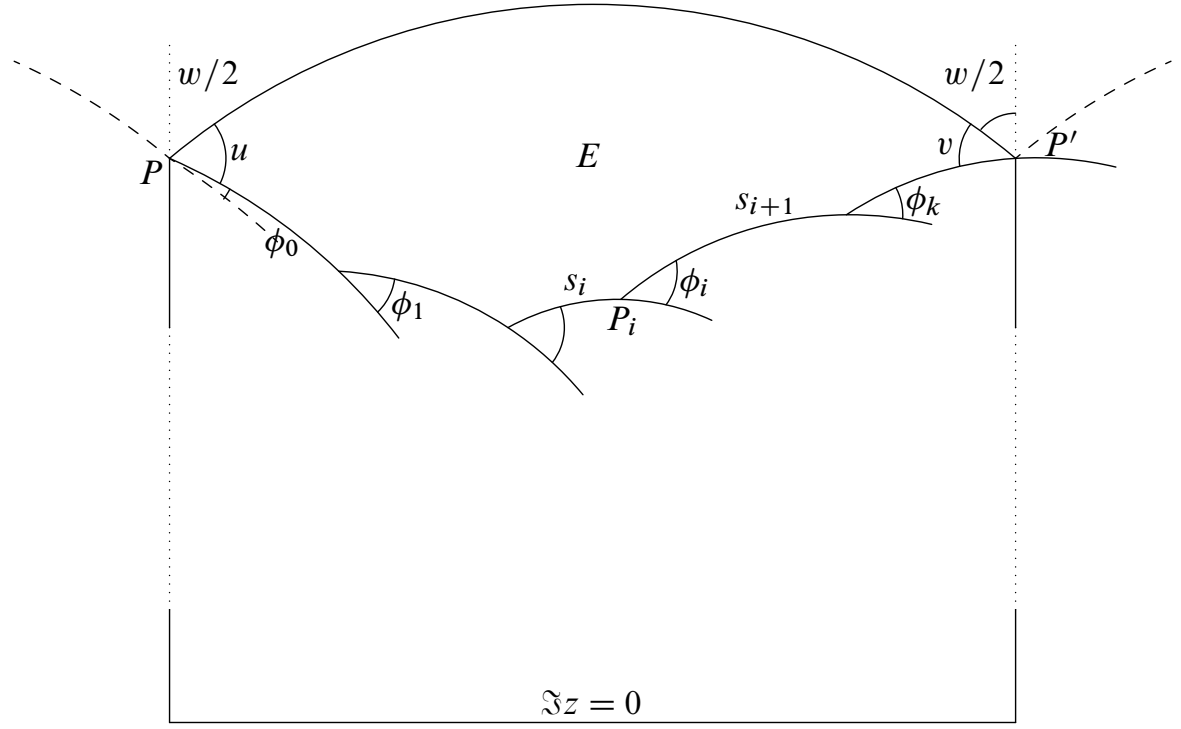

Figure 8: The geodesic representative $\widetilde{S}^{+}$of $\sigma$ on $\partial \mathcal{C}^{+}$

Combining (2), (4) and (3), we find

$$
\pi-w<\sum_{0}^{k} \phi_{i} \leq \theta i(\sigma, \xi) .
$$

Now the angles in $\Delta$ at $P$ and $P^{\prime}$ are both $w / 2$, so by the angle of parallelism formula, writing $d=d_{\mathbb{H}^{3}}\left(P, P^{\prime}\right)$,

$$
\sinh d / 2=\cot w / 2,
$$

so that from (5) we find

$$
\sinh d / 2 \leq \theta i(\sigma, \xi)\left(1+O\left(\theta^{2}\right)\right) / 2 .
$$

Finally, we claim that

$$
l_{\sigma}^{+} \leq d\left(1+O\left(\theta^{2}\right)\right)
$$

for small $\theta$, from which the result is immediate. This follows easily from Lemma 6.3. We have only to see that the angle $\psi$ between the line $P X$ from $P$ to any point $X$ on $\widetilde{S}^{+}$is bounded above by $\theta i(\sigma, \xi)$. This follows since along the interior of any segment $\psi$ decreases as $x$ increases, and since at the bend point $P_{i}$ it increases by at most $\theta_{i}$. This gives $d \geq l_{\sigma}^{+}\left(1-O\left(\theta^{2}\right)\right)$ and the result follows. 
Corollary 6.5 Suppose that $\xi \in M L_{\mathbb{Q}}$ is admissible and suppose that $G\left(\tau_{1}, \tau_{2}\right)$ is the unique group $G_{\xi}(\theta)$ in $\mathcal{M}$ with $\beta(G)=\theta \xi$. Then $1 / \Im \tau_{i} \leq O(\theta), i=1,2$, as $\theta \rightarrow 0$. Moreover the groups $G_{\xi}(\theta)$ have no algebraic limit as $\theta \rightarrow 0$.

Proof As above, we work in the upper half space model and we assume $G=G_{\xi}(\theta)$ normalised so that $S=S_{i}$ is the translation $z \mapsto z+2$. Define $P, P^{\prime}$ as before. Let $k$ be the Euclidean height $k$ of $P$ above $\mathbb{C}$. Then $1 / k=\sinh d_{\mathbb{H}^{2}}\left(P, P^{\prime}\right) / 2$ while on the other hand, $d_{\mathbb{H}^{2}}\left(P, P^{\prime}\right) \leq l_{\sigma^{+}} \leq O(\theta)$. It follows that $1 / k \leq O(\theta)$.

Now $P$ lies on a bending line $\zeta$ of $\partial \mathcal{C}^{+}$which is contained in a support plane to $\partial \mathcal{C}^{+}$. This plane is a hemisphere $H \subset \mathbb{H}^{3}$ which meets $\widehat{\mathbb{C}}$ in a circle $C$. Since the convex core is contained entirely to one side of $H$, there are no limit points in one of the two discs in $\widehat{\mathbb{C}}$ bounded by $C$. Since the horizontal lines $\Im z=0$ and $\Im z=\Im \tau_{i}$ are contained in the limit set $\Lambda$, and since the half planes $\Im z<0$ and $\Im z>\Im \tau_{i}$ are contained in $\Omega^{-}$, it follows that $C$ is contained in $\left\{z: 0 \leq \Im z \leq \Im \tau_{i}\right\}$ and hence that its diameter is at most $\Im \tau_{i}$. We deduce $2 / \Im \tau_{i} \leq 1 / k$ so that $1 / \Im \tau_{i} \leq O(\theta)$ as claimed.

To prove that the algebraic limit of a sequence of groups does not exist, it is enough to show that the trace of some element becomes infinite. The result follows on recalling from Section 2.2 that $\operatorname{Tr}\left[T, S_{i}^{-1}\right]=\tau_{j}^{2}+2, j \neq i \bmod 2$.

Now we establish a more precise link between the length $l_{\sigma_{i}}^{+}$of $\sigma_{i}$ on $\partial \mathcal{C}^{+}$and $\Im \tau_{i}$ :

Proposition 6.6 Let $\xi \in M L_{\mathbb{Q}}$ be admissible and suppose that $G\left(\tau_{1}, \tau_{2}\right)$ is the unique group $G_{\xi}(\theta) \in \mathcal{M}$ with $\beta(G)=\theta \xi$. Then

$$
\Im \tau_{i}(1-O(\theta)) \leq 4 / l_{\sigma_{i}}^{+} \leq \Im \tau_{i}(1+O(\theta))
$$

as $\theta \rightarrow 0$.

Remark 6.7 Kra [17, page 568] gives the estimate $\Im \tau_{i}-1 \leq 2 \pi / l_{\sigma_{i}}\left(\Omega^{+}\right) \leq \Im \tau_{i}$ for the hyperbolic length $l_{\sigma_{i}}\left(\Omega^{+}\right)$of the geodesic representative of $\sigma$ on $\Omega^{+} / G$. Combining this with Sullivan's theorem [10], gives an alternative proof of the final statement of Corollary 6.5. The discrepancy of the $\pi / 2$ factor with the estimate in Proposition 6.6 is perhaps somewhat surprising, as one would expect that, since $G$ becomes asymptotically Fuchsian, the structures on $\partial \mathcal{C}^{+} / G$ and $\Omega^{+} / G$ would be asymptotically equal. For a quick confirmation of Kra's estimate, note that $\Omega^{+}$ contains an $S$ invariant strip of width $\Im \tau-1$, so that there is an annular collar $A$ of approximate modulus $\Im \tau / 2$ around $S$ on $\Omega^{+}$. Kra's estimate follows from the formula $\bmod A=\pi / l_{\sigma}\left(\Omega^{+}\right)+O(1)$; see for example Minsky [23, page 255].

We begin the proof of Proposition 6.6 with two lemmas. 
Lemma 6.8 Let $\xi \in M L_{\mathbb{Q}}$ be admissible. Normalize $G_{\xi}(\theta)$ so that $S_{i}$ is translation $z \mapsto z+2$. Let $\gamma$ be a bending line of the pleating lamination $\xi$ which intersects $\sigma_{i}$. Then there is a lift $\tilde{\gamma}$ of $\gamma$ with endpoints $\gamma^{ \pm}$such that $\left|\Re\left(\gamma^{+}-\gamma^{-}\right)\right| \leq 2$ and $\mathfrak{s} \tau_{i}-1<\left|\Im\left(\gamma^{+}-\gamma^{-}\right)\right|<\mathfrak{s} \tau_{i}$.

Proof We continue with the set up of Corollary 6.5. We can choose a lift $\tilde{\gamma}$ of $\gamma$ to be identified with the bending line $\zeta$ in that proof. The endpoints $\gamma^{ \pm}$of $\tilde{\gamma}$ lie in $C \cap \Lambda$, where $C$ is a circle contained in the strip $B$ between the lines $\Im z=0$ and $\Im z=\Im \tau_{i}$ and as usual $\Lambda$ is the limit set of $G$. By Proposition 2.1, $\Lambda$ is contained in the union of

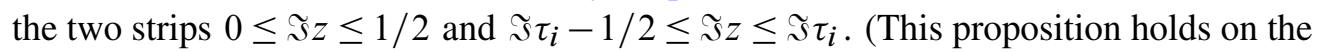
hypothesis that $\Im \tau_{i}>1$, which holds for small $\theta$ by Corollary 6.5.) Moreover there are no points of $\Lambda$ in the intersection of the two open disks $D$ and $S(D)$ bounded by $C$ and $S(C)$ and inside $B$. We deduce that $\zeta$ either has Euclidean height at most $O(1)$, or has endpoints one of which is in the rectangle $|\Re z|<1,0 \leq \Im z \leq 1 / 2$ and the other

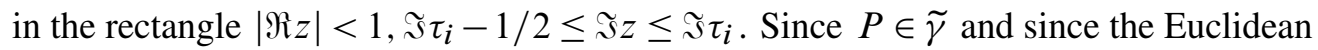
height of $P$ is large by Corollary 6.5, the result follows.

Continuing with the assumption that $\xi \in M L_{\mathbb{Q}}$ is admissible and that $G_{\xi}(\theta)$ is normalized so that $S_{i}$ is translation $z \mapsto z+2$, we call any lift of a bending line satisfying the conditions of Lemma 6.8, good. Combining with Corollary 6.5 we obtain easily:

Corollary 6.9 Let $\tilde{\gamma}$ be a good lift of a bending line $\gamma$ and set $\gamma^{+}-\gamma^{-}=2 r e^{i \alpha}$, where without loss of generality we take $\Im\left(\gamma^{+}-\gamma^{-}\right)>0$. Then $r=(1+O(\theta)) \Im \tau_{i} / 2$ and $|\pi / 2-\alpha|=O(\theta)$ as $\theta \rightarrow 0$.

Lemma 6.10 Let $\tilde{\gamma}$ be a good lift of a bending line of the pleating lamination which intersects $\widetilde{S}_{i}^{+}$. Then the complex distance $D$ between $\tilde{\gamma}$ and $S_{i}(\tilde{\gamma})$ is given by

$$
-\frac{1}{r^{2} e^{2 i \alpha}}=\sinh ^{2} D / 2 \text {. }
$$

Proof Recall that the complex distance between two axes is $d+i \psi$ where $d$ is the real perpendicular distance and $\psi$ is the rotation of one axis relative to the other along their common perpendicular; see eg Series [27] for details. We use the cross ratio formula for complex distance. Let $z_{1}, z_{2}$ and $w_{1}, w_{2}$ be endpoints of two oriented geodesics in $\mathbb{H}^{3}$ at complex distance $D$. We can conjugate so that $z_{1}, z_{2}$ move to $1,-1$ and $w_{1}, w_{2}$ move to $e^{D},-e^{D}$ respectively. Then

$$
\left[z_{1}, w_{1}, w_{2}, z_{2}\right]:=\frac{z_{1}-w_{2}}{z_{1}-w_{1}} \cdot \frac{w_{1}-z_{2}}{w_{2}-z_{2}}=\operatorname{coth}^{2} D / 2 .
$$


Applying this formula to the four endpoints of $\tilde{\gamma}, S_{i}(\tilde{\gamma})$ which are at points $\gamma^{+}, \gamma^{-}$ and $\gamma^{+}+2, \gamma^{-}+2$ respectively gives

$$
\operatorname{coth}^{2} D / 2=\left[\gamma^{+}, \gamma^{+}+2, \gamma^{-}+2, \gamma^{-}\right]
$$

which simplifies to the claimed result.

Proof of Proposition 6.6 Pick a good lift $\tilde{\gamma}$ of a bending line, and let $P$ be the point where $\tilde{\gamma}$ meets $\tilde{S}^{+}$. Let $Q$ be the highest point of $\tilde{\gamma}$ and let $Q^{\prime}=S(Q)$, so that the Euclidean height of both $Q, Q^{\prime}$ above $\mathbb{C}$ is $r$. Writing $d_{\partial \mathcal{C}}$ for the induced hyperbolic metric on $\partial \mathcal{C}^{+}$, we have

$$
l_{\sigma^{+}} \leq d_{\partial \mathcal{C}}\left(Q, Q^{\prime}\right) \leq d_{\mathbb{H}^{3}}\left(Q, Q^{\prime}\right)\left(1+O\left(\theta^{2}\right)\right)
$$

where the first inequality follows since the curve on $\partial \mathcal{C}^{+}$from $Q$ to $Q^{\prime}$ is in the homotopy class of $\widetilde{S}^{+}$, and the final one follows by Lemma 6.3 .

Now

$$
\sinh d_{\mathbb{H}^{3}}\left(Q, Q^{\prime}\right) / 2=1 / r
$$

and $\Im \tau-1 \leq 2 r \leq \Im \tau$ by Lemma 6.8. Thus

$$
d_{\mathbb{H}^{3}}\left(Q, Q^{\prime}\right) / 2 \leq 1 / r \leq 2 /(\Im \tau-1)
$$

from which we deduce

$$
l_{\sigma^{+}} \leq 4 \frac{\left(1+O\left(\theta^{2}\right)\right)}{\Im \tau-1}=\frac{4}{\Im \tau}(1+O(1 / \Im \tau))\left(1+O\left(\theta^{2}\right)\right)=\frac{4}{\Im \tau}(1+O(\theta))
$$

by Corollary 6.5. Hence

$$
\Im \tau / 4 \leq 1 / l_{\sigma^{+}}(1+O(\theta)) .
$$

To find an upper bound for $1 / l_{\sigma^{+}}$, we use Lemma 6.10. Writing $D=d+i \psi$, note that since $d \leq l_{\sigma^{+}}$it is enough to find an upper bound on $1 / d$. Comparing real and imaginary parts in the formula of Lemma 6.10 gives

$$
\sinh d / 2 \cos \psi / 2= \pm \sin \alpha / r \quad \text { and } \quad \cosh d / 2 \sin \psi / 2= \pm \cos \alpha / r .
$$

Since $d \leq l_{\sigma^{+}} \leq O(\theta)$ we find

$$
\frac{2}{d} \leq \frac{\left(1+O\left(\theta^{2}\right)\right)}{\sinh d / 2}=\frac{r\left(1+O\left(\theta^{2}\right)\right)}{|\sin \alpha|}|\cos \psi / 2|
$$

By Corollary 6.9, we have $1 /|\sin \alpha|=1+O(\theta)$. Thus

$$
2 / d \leq r(1+O(\theta))
$$


from which

$$
2 / l_{\sigma^{+}} \leq \Im \tau(1+O(\theta)) / 2 .
$$

Inequalities (6) and (7) together complete the proof.

\subsection{Asymptotic orthogonality}

Propositions 6.4 and 6.6 are not enough to give the detailed asymptotics of Theorem 6.1. We also need the following more refined comparison:

Proposition 6.11 Along the pleating variety $\mathcal{P}_{\xi}$, we have

$$
\theta i(\xi, \sigma)(1-O(\theta)) \leq l_{\sigma}^{+} \leq \theta i(\xi, \sigma)\left(1+O\left(\theta^{2}\right)\right)
$$

as $\theta \rightarrow 0$.

This result is a direct consequence of the fact that asymptotically, the lift $\widetilde{S}^{+}$of the geodesic representative of $S$ on $\partial^{+}$becomes orthogonal to the bending lines; see Proposition 6.13. The intuition for this is the following. Suppose that $\widetilde{S}^{+}$were actually perpendicular to all bending lines. Then each good lift of a bending line cut by $\widetilde{S}^{+}$would have Euclidean height between $\Im \tau / 2-1$ and $\Im \tau / 2$, and in the proof of Proposition 6.4, Equations (2) and (3) would be equalities. Since $E$ has area $O\left(\theta^{2}\right)$, in this situation (5) becomes an equality up to $O\left(\theta^{2}\right)$. This situation actually pertains in the case in which there is unique bending line in the class of $T$ (in which case $E=\varnothing$ ); see Example 1 in Section 5.

Lemma 6.12 Let $\tilde{\gamma}$ be a good lift of a bending line and as above, let $P, P^{\prime}$ be the points at which $\widetilde{S}^{+}$meets $\tilde{\gamma}$ and $S(\tilde{\gamma})$ respectively. If $K$ is the Euclidean centre on $\mathbb{C}$ of the semicircle $\tilde{\gamma}$, then $\angle P K Q=O(\sqrt{\theta})$.

Proof As in Corollary 6.5, let $k$ denote the Euclidean height of $P$. We have $\sinh d_{\mathbb{H}^{3}}\left(P, P^{\prime}\right) / 2=1 / k$ and $1 / k<O(\theta)$. Hence $d_{\mathbb{H}^{3}}\left(P, P^{\prime}\right)=2\left(1+O\left(\theta^{2}\right)\right) / k$. We will estimate $k / r=\cos \angle P K Q$.

By Lemma 6.3

$$
d_{\mathbb{H}^{3}}\left(P, P^{\prime}\right) \leq l_{\sigma^{+}} \leq\left(1+O\left(\theta^{2}\right)\right) d_{\mathbb{H}^{3}}\left(P, P^{\prime}\right)
$$

and hence

$$
l_{\sigma^{+}}=2\left(1+O\left(\theta^{2}\right)\right) / k .
$$

On the other hand, by Proposition 6.6 and Lemma 6.8,

$$
2 / l_{\sigma^{+}}=r(1+O(\theta)) .
$$


Combining these two equations gives

$$
|k / r-1|=O(\theta)
$$

so that $\angle P K Q \leq O(\sqrt{\theta})$ as claimed.

Now we prove our result on asymptotic orthogonality.

Proposition 6.13 Along the pleating variety $\mathcal{P}_{\xi}$, the curve $\widetilde{S}^{+}$is asymptotically orthogonal to the bending lines as $\theta \rightarrow 0$. More precisely, suppose that $\widetilde{S}^{+}$meets an (oriented) bending line $\tilde{\gamma}$ at a point $P$ so that the acute angle between $\widetilde{S}^{+}$and $\tilde{\gamma}$ is $\psi(P)$. Then $|\psi(P)-\pi / 2| \leq O(\sqrt{\theta})$ as $\theta \rightarrow 0$.

Proof As usual, we may suppose that $\tilde{\gamma}$ is a good lift of a bending line, and we let $P^{\prime}=S(P)$. Let $\kappa$ denote the geodesic arc in $\mathbb{H}^{3}$ from $P$ to $P^{\prime}$. Let $\mathbf{u}, \mathbf{v}, \mathbf{w}$ be forward pointing unit vectors at $P$ along $\tilde{S}^{+}, \kappa$, and $\tilde{\gamma}$ respectively, where $\kappa, \widetilde{S}^{+}$ are given the same orientation and the orientation of $\tilde{\gamma}$ is from $\gamma^{-}$to $\gamma^{+}$, where $\Im \gamma^{-}<\Im \gamma^{+}$. Let $\Psi(\mathbf{x}, \mathbf{y})$ denote the angle between the vectors $\mathbf{x}$ and $\mathbf{y}$ at $P$, so that $\psi(P)=\Psi(\mathbf{u}, \mathbf{w})$.

From Equation (4) in the proof of Proposition 6.4, we have $\Psi(\mathbf{u}, \mathbf{v}) \leq \theta i(\sigma, \xi)$. Thus it will suffice to show that $|\Psi(\mathbf{v}, \mathbf{w})-\pi / 2|=O(\sqrt{\theta})$. We prove this by a finding a sequence of small rotations which, when applied to $\mathbf{v}$ and $\mathbf{w}$, results in a pair of perpendicular vectors.

Let $\Gamma$ be the vertical plane containing $\tilde{\gamma}$ and let $Z$ be the footpoint of the perpendicular from $P$ to $\mathbb{C}$. As in Lemma 6.12 , let $K$ be the footpoint of the perpendicular from the highest point $Q$ on $\tilde{\gamma}$ to $\mathbb{C}$. Let $\Theta_{1}$ be rotation by an angle $\angle P K Q$ about a line perpendicular to $\Gamma$ through $P$, so that $\Theta_{1}(\mathbf{w})$ is horizontal (ie parallel to the base plane $\mathbb{C})$. By Lemma 6.12, $\angle P K Q=O(\sqrt{\theta})$, so that $\Theta_{1}(\mathbf{w})=\mathbf{w}+O(\sqrt{\theta})$.

The vectors $\mathbf{w}$ and $\Theta_{1}(\mathbf{w})$ are in the plane $\Gamma$ containing $\gamma$, which is at angle $\alpha$ to the real axis and hence to the vertical plane $\Pi$ containing $P$ and $P^{\prime}$. These two planes intersect in the line $Z P$. Thus if $\Theta_{2}$ denotes anticlockwise rotation by $\pi / 2-\alpha$ about $Z P$, then $\Theta_{2}\left(\Theta_{1}(\mathbf{w})\right)$ is orthogonal to $\Pi$ at $P$. Since by Lemma $6.8, \pi / 2-\alpha=O(\theta)$, we have $\mathbf{w}=\Theta_{2}\left(\Theta_{1}(\mathbf{w})\right)+O(\sqrt{\theta})$.

Finally, let $\zeta$ be the angle between $P Z$ and the arc $\kappa$ at $P$, so that $\sinh d_{\mathbb{H}^{3}}\left(P, P^{\prime}\right) / 2=$ $\cot \zeta$. Since $d_{\mathbb{H}^{3}}\left(P, P^{\prime}\right) \leq l_{\sigma^{+}}$this gives $\pi / 2-\zeta=O(\theta)$. If $\Theta_{3}$ is rotation by an angle $\pi / 2-\zeta$ about a line perpendicular to $\Pi$ through $P$, then $\Theta_{3}(\mathbf{v})$ is horizontal and lies in $\Pi$ and $\Theta_{3}(\mathbf{v})=\mathbf{v}+O(\theta)$.

By construction $\Theta_{2}\left(\Theta_{1}(\mathbf{w})\right)$ is orthogonal to $\Theta_{3}(\mathbf{v})$, so that putting these three estimates together we find $|\Psi(\mathbf{v}, \mathbf{w})-\pi / 2|=O(\sqrt{\theta})$ as claimed. 
Finally, we can prove Proposition 6.11.

Proof of Proposition 6.11 In view of Proposition 6.4, it will be enough to find a bound $l_{\sigma^{+}} \geq \theta i(\xi, \sigma)(1-O(\theta))$ as $\theta \rightarrow 0$.

We work in the vertical plane $\Pi$ containing $P$ and $P^{\prime}$. This plane intersects $\partial \mathcal{C}^{+}$in a path which is a union of $\mathbb{H}^{3}$-geodesic segments similar to but not the same as the geodesic path $s_{0}, s_{1}, \ldots, s_{k}$ of Proposition 6.4. Denote the bending points along this path $P=\widehat{P}_{0}, \widehat{P}_{1}, \ldots, \widehat{P}_{k+1}$ where $\widehat{P}_{k+1}=S\left(P_{0}\right)=P^{\prime}$, and let $\widehat{s}_{i}$ be the segment from $\widehat{P}_{i-1}$ to $\hat{P}_{i}$.

Let $\hat{\phi}_{i}$ denote the exterior angle between the segments $\widehat{s}_{i}, \widehat{s}_{i+1}$ which meet at $\widehat{P}_{i}$, measured in the same way as $\phi$ in the proof of Proposition 6.4, and let $\theta_{i}$ be the bending angle between the support planes of $\partial \mathcal{C}^{+}$which meet at $\hat{P}_{i}$. We will prove below that

$$
\widehat{\phi}_{i} / \theta_{i}=1+O(\theta)
$$

Denote by $l\left(\widehat{s}_{i}\right)$ the hyperbolic length of $\hat{s}_{i}$. We observe

$$
l_{\sigma}^{+} \geq d_{\mathbb{H}^{3}}\left(P, P^{\prime}\right) \geq\left(1-O\left(\theta^{2}\right)\right) \sum l\left(\hat{s}_{i}\right)
$$

where the last inequality follows by Lemma 6.3 since from (8) $\hat{\phi}_{i}=O(\theta)$ for all $i$. To estimate $l\left(\widehat{s}_{i}\right)$, join each point $\widehat{P}_{i}$ to $\infty$ in the plane $\Pi$ and let $y_{i}=\angle \widehat{P}_{i-1} \widehat{P}_{i} \infty$ and $x_{i}=$ $\angle \infty \hat{P}_{i} \hat{P}_{i+1}$, so that (since all angles are measured in the plane $\Pi$ ), $x_{i}+y_{i}+\hat{\phi}_{i}=\pi$. The formula for the length of the finite side of triangle with angles $x, y, 0$ (see Beardon [3, Theorem 7.10.1]) gives

$$
\sinh l\left(\widehat{s}_{i}\right)=\frac{\cos x_{i}+\cos y_{i+1}}{\sin x_{i}+\sin y_{i+1}} .
$$

We also prove below that

$$
\left|\pi / 2-x_{i}\right|=O(\theta) \quad \text { and } \quad\left|\pi / 2-y_{i}\right|=O(\theta) .
$$

This gives

$$
l\left(\hat{s}_{i}\right)=\left(\pi-x_{i}-y_{i+1}\right)\left(1+O\left(\theta^{2}\right)\right)
$$

from which

$$
\sum_{0}^{k} l\left(\widehat{s}_{i}\right)=\left(\sum_{0}^{k} \hat{\phi}_{i}\right)\left(1+O\left(\theta^{2}\right)\right)=\left(\sum_{0}^{k} \theta_{i}\right)(1+O(\theta))
$$

by (8). Hence

$$
l_{\sigma}^{+} \geq \theta i(\xi, \sigma)(1-O(\theta))
$$

as $\theta \rightarrow 0$. This completes the proof, modulo the proofs of (8) and (9). 
Proof of (8) This follows from Appendix A.4 in [10]. Suppose that two planes $J_{1}, J_{2}$ meet at an angle $\zeta$ along a line $L$, so that $\zeta$ is the angle between the lines of intersection of $J_{1}, J_{2}$ with the plane $H$ orthogonal to $L$. Suppose that $H^{\prime}$ is another plane slightly skewed to $H$, and let $\zeta^{*}$ be the angle between the lines of intersection of $J_{1}, J_{2}$ with $H^{\prime}$. Setting things up so that $H$ has unit normal $(0,0,1)$ and so that the bisector of the planes orthogonal to $L$ is the vector $(1,0,0)$, the result of [10] gives an estimate of $\zeta^{*} / \zeta$ in terms of the unit normal $\left(x_{1}, x_{2}, x_{3}\right)$ to $H^{\prime}$ for small $x_{1}, x_{2}$. In fact if $x_{i}=O(\epsilon), i=1,2$, then we find easily either from the formula for $\tan \zeta^{*} / 2 / \tan \zeta / 2$ in terms of the $x_{i}$ on page 246, or from Theorem A.4.2 on page 247, that $\zeta^{*} / \zeta=1+O\left(\epsilon^{2}\right)$.

To apply the theorem in our case, we want to find the angle between the support planes which meet along the bending line at $P_{i}$, measured in the plane $\Pi$ which is slightly skewed to the plane orthogonal to the lift $\tilde{\gamma}_{i}$ of $\gamma$ through $\widehat{P}_{i}$. In the above set up, the unit vector along $\tilde{\gamma}$ at $\hat{P}_{i}$ is $\mathbf{e}_{3}=(0,0,1)$, and the line bisecting the planes along $\gamma$ is $\mathbf{e}_{\mathbf{1}}=(1,0,0)$.

Let $\mathbf{e}_{\mathbf{3}}^{\prime}, \mathbf{e}_{\mathbf{1}}^{\prime}$ be unit vectors at $\hat{P}_{i}$ orthogonal to $\Pi$, and in $\Pi$ pointing vertically upwards, respectively. It will be sufficient to show that $\mathbf{e}_{\mathbf{3}}^{\prime}=\mathbf{e}_{\mathbf{3}}+O(\sqrt{\theta})$ and $\mathbf{e}_{\mathbf{1}}^{\prime}=\mathbf{e}_{\mathbf{1}}+O(\sqrt{\theta})$. Now if $\widehat{P}_{i}$ were replaced by the similar configuration at the point $P_{i}$, then the first result would follow from Corollary 6.9 and Lemma 6.12, while the second would follow from the proof of Proposition 6.4, since as illustrated in Figure 8, $\mathbf{e}_{\mathbf{1}}^{\prime}$ bisects the angle between $s_{i}$ and $s_{i+1}$ up to $O(\theta)$.

In the move from $P_{i}$ to $\hat{P}_{i}$, the estimates for $\mathbf{e}_{\mathbf{3}}^{\prime}, \mathbf{e}_{\mathbf{1}}^{\prime}$ will change by terms on the order of $\angle P_{i} K_{i} \widehat{P}_{i}$, the angular distance from $P_{i}$ to $\widehat{P}_{i}$ along $\tilde{\gamma}_{i}$. (Here $K_{i}$ is the centre of the Euclidean semicircle $\tilde{\gamma}_{i}$.) We estimate this as follows. Let $d_{i}$ be the perpendicular distance between the plane $\Pi$ through $P$ parallel to the real axis, and the parallel plane $\Pi_{i}$ through $P_{i}$. Since $P$ is joined to $P_{i}$ by segments $s_{0}, s_{1}, \ldots s_{i-1}$ along $\widetilde{S}^{+}$, and since it follows from (4) in Proposition 6.4 that each segment $s_{j}$ makes an angle at most $O(\theta)$ with the plane $P_{j-1}$, we find

$$
d_{i} \leq \sum_{0}^{i-1} l\left(s_{j}\right) O(\theta)=O\left(\theta^{2}\right) .
$$

Let $Z_{i}, \hat{Z}_{i}$ denote the footpoints in $\mathbb{C}$ of the vertical lines through $P_{i}, \hat{P}_{i}$. The estimate on $d_{i}$ combined with Corollary 6.9 gives $\left|Z_{i}-\hat{Z}_{i}\right|=O\left(\theta^{2}\right)$. It follows that the error in replacing $P_{i}$ by $\widehat{P}_{i}$ is of a lower order than those already obtained, and we conclude that $\mathbf{e}_{\mathbf{3}}^{\prime}=\mathbf{e}_{\mathbf{3}}+O(\sqrt{\theta})$ and $\mathbf{e}_{\mathbf{1}}^{\prime}=\mathbf{e}_{\mathbf{1}}+O(\sqrt{\theta})$ as claimed.

Proof of (9) Let $L$ be a line from $\infty$ to a variable point $X$ on some segment $\widehat{s}_{i}$, and let $x=x(X)>0$ be the acute angle in the complementary pair $\angle \widehat{P}_{i-1} X \infty$ and 
$\angle \infty X \hat{P}_{i}$. We want to show that $|\pi / 2-x|=O(\theta)$. Since $P_{0}$ and $S\left(P_{0}\right)$ are at the same Euclidean height, there is certainly some $\widehat{s}_{i_{0}}$ containing a point $X_{0}$ at which the tangent to $\hat{s}_{i_{0}}$ is horizontal, so $x\left(X_{0}\right)=\pi / 2$. Moving away from this point in either direction, $x(X)$ decreases according to the formula $\sinh t=\cot x(X(t))$, where the point $X(t)$ is at distance $t$ from $X_{0}$ along $\widehat{s}_{i_{0}}$. The change in angle at a bend point is $\hat{\phi}_{i}=\theta_{i}(1+O(\theta))$ by (8), and on the adjacent segment the argument proceeds as before. Since (using Lemma 6.3 again) $\sum_{i} l\left(\hat{s}_{i}\right) \leq O(\theta)$, the result follows.

\subsection{Twisting}

To complete the proof of Theorem 6.1, it remains to bound $\Re \tau_{i}$. We have:

Proposition 6.14 Let $\xi \in M L_{\mathbb{Q}}$ be admissible and suppose that $\gamma \in \mathcal{S}$ is contained in the support of $\xi$. Then if $\left(\tau_{1}, \tau_{2}\right) \in \mathcal{P}_{\xi}$, we have $\Re \tau_{i}=-2 p_{i}(\gamma) / q_{i}(\gamma)+O(1)$ and hence in particular $\left|\arg \tau_{i}-\pi / 2\right|=O(\theta)$ as $\theta \rightarrow 0$.

Since this result holds for any $\gamma$ contained in the support of $\xi$ we have:

Corollary 6.15 Suppose that $\gamma, \gamma^{\prime} \in \mathcal{S}$ are supported on a common admissible lamination $\xi$. Then $\left|p_{i}(\gamma) / q_{i}(\gamma)-p_{i}\left(\gamma^{\prime}\right) / q_{i}\left(\gamma^{\prime}\right)\right| \leq 10$, hence $\Re \tau_{i}=-2 p_{i}(\xi) / q_{i}(\xi)+O(1)$.

The condition that $\gamma, \gamma^{\prime}$ are supported on a common admissible lamination is equivalent to the condition that together with $\sigma_{1}, \sigma_{2}$ they fill up $\Sigma$. The value 10 is obtained by following through the constants in the argument below; it could certainly be improved by more careful inspection.

We prove this result using the concept of the twist of one geodesic around another following Minsky [23]. Suppose given a hyperbolic metric $h$ on the surface $\Sigma$. The twist $\operatorname{tw}_{\beta}(\gamma, h)$ of a curve $\gamma$ about another curve $\beta$ is defined as follows. Let $p$ be an intersection point of $\gamma$ with $\beta$. Let $P$ be a lift to $\mathbb{H}^{2}$ of $p$ and let $\tilde{\gamma}, \tilde{\beta}$ be the lifts of $\gamma, \beta$ through $P$. Orient $\tilde{\gamma}, \widetilde{\beta}$ with positive endpoints $Z, W$ respectively on $\partial \mathbb{H}^{2}$ so that the anticlockwise arc from $Z$ to $W$ does not contain the other two endpoints. Let $R$ be the footpoint of the perpendicular from $Z$ to $\tilde{\beta}$. Let $t$ be the oriented distance $P R$, where $t>0$ if $R$ follows $P$ in the positive direction along $\tilde{\beta}$ and $t \leq 0$ otherwise. One verifies [23, Lemma 3.1] that $t / l_{\beta}(h)$ is independent up to an additive error of 1 of the choices made, including the choice of $p$. Finally, define $\operatorname{tw}_{\beta}(\gamma, h)=2 \inf t / l_{\beta}(h)$, where we take the infimum over all possible choices of lifts as above.

Note that the twist is independent of the orientation of $\beta, \gamma$ but depends on the choice of hyperbolic metric $h \in \mathcal{T}$, where $\mathcal{T}$ is the Teichmüller space of $\Sigma$. However: 
Lemma 6.16 ([23, Lemma 3.5]; see also [8, Section 4.3]) For any two $\gamma_{1}, \gamma_{2} \in \mathcal{S}$, the relative twist $\operatorname{tw}_{\alpha}\left(\gamma_{1}, h\right)-\operatorname{tw}_{\alpha}\left(\gamma_{2}, h\right)$ is independent of $h \in \mathcal{T}$, up to a bounded additive error of 1 .

We define the signed relative twist of $\gamma_{1}, \gamma_{2}$ with respective to $\beta$ to be $i_{\beta}\left(\gamma_{1}, \gamma_{2}\right)=$ $\inf _{h \in \mathcal{T}} \operatorname{tw}_{\beta}\left(\gamma_{1}, h\right)-\operatorname{tw}_{\beta}\left(\gamma_{2}, h\right)$. Here is a useful way of computing it:

Lemma 6.17 Let $\gamma_{1}, \gamma_{2} \in \mathcal{S}$ and let $\tilde{\gamma}_{1}, \tilde{\gamma}_{2}$ be lifts of $\gamma_{1}, \gamma_{2}$ which cut the fixed axis $\tilde{\beta}$ corresponding to $\beta$, and let $b \in \Gamma$ be the primitive element whose axis is $\tilde{\beta}$ and whose attracting fixed point is the positive endpoint of $B$, where $\Gamma$ is the Fuchsian group uniformising $h$. Then $\operatorname{tw}_{\beta}\left(\gamma_{1}, h\right)-\operatorname{tw}_{\beta}\left(\gamma_{2}, h\right)$ is equal in magnitude to the number of times the images $b^{n}\left(\tilde{\gamma}_{1}\right), n \in \mathbb{Z}$ intersect $\widetilde{\gamma}_{2}$, up to a bounded additive error of 1 . The sign is negative if $b\left(\tilde{\gamma}_{1}\right)$ follows $\tilde{\gamma}_{1}$ in the positive direction along $\tilde{\gamma}_{2}$ and positive otherwise.

Proof This is clear from the definition in a metric in which $\tilde{\gamma}_{1}$ is orthogonal to $B$. Whether or not two axes intersect, depends only on the relative position of their endpoints round the boundary at infinity $\partial \mathbb{H}=S^{1}$. Since a quasiconformal deformation of $\mathbb{H}$ induces a homeomorphism of $\partial \mathbb{H}$, we deduce that the relative positions of endpoints of axes are independent of the metric $h$, from which the result follows.

We shall prove Proposition 6.14 by computing $i_{\sigma_{i}}(\gamma, T)$ in two different ways, where as usual $i_{\sigma_{i}}(\gamma, T)$ means $i_{\sigma_{i}}\left(\gamma, \gamma_{T}\right)$ where $\gamma_{T} \in \mathcal{S}$ is the curve corresponding to the generator $T \in \pi_{1}(\Sigma)$. We have:

Lemma 6.18 Suppose that $\gamma \in \mathcal{S}$ has canonical coordinates

$$
\mathbf{i}(\gamma)=\left(q_{1}(\gamma), p_{1}(\gamma), q_{2}(\gamma), p_{2}(\gamma)\right)
$$

Then $i_{\sigma_{i}}(\gamma, T)=-p_{i}(\gamma) / q_{i}(\gamma)+O(1)$.

Proof We work in the fundamental domain $\Delta$ of $\Sigma_{1,2}$ and label the sides as in Figure 2. We also suppose that $p_{1} \geq 0$. Let $\tilde{A}$ be a horizontal strip joining $s_{S_{1}}$ to $s_{S_{1}^{-1}}$, shown shaded in the figure. This projects to an annular neighbourhood $A$ of $\sigma_{1}$ on $\Sigma$. We may take our lift $\widetilde{\sigma}_{1}$ of $\sigma_{1}$ to be the centre line of $\tilde{A}$ and the lift $\tilde{\gamma}_{T}$ of the curve $\gamma_{T}$ to be the arc joining the midpoints of $s_{T}$ and $s_{T^{-1}}$. This intersects $\tilde{A}$ in a single arc $\lambda$ which joins the boundaries $\partial^{-} \tilde{A}, \partial^{+} \tilde{A}$ of $\tilde{A}$. By the previous lemma, to compute $i_{\sigma_{1}}(\gamma, T)$ we have to examine how many images $S_{1}^{n}\left(\tilde{\gamma}_{T}\right)$ cut a fixed lift $\tilde{\gamma}$ of $\gamma$, equivalently how many images $S_{1}^{n}(\tilde{\gamma})$ cut $\tilde{\gamma}_{T}$. 
The lift $\tilde{\gamma}$ appears as a collection of disjoint arcs joining sides of $\Delta$, the numbers of arcs joining particular pairs of sides being determined by the canonical coordinates $\mathbf{i}(\gamma)$. It is not hard to see that the magnitude of the relative intersection number $i_{\sigma_{i}}(\gamma, T)$ is, up to an additive error of 1 , the number of times that a connected component $\kappa$ of $\gamma \cap A$ cuts the projection $\pi(\lambda)$ of $\lambda$ to $\Sigma$. For convenience, replace $\lambda$ by $L=s_{S_{1}} \cap \tilde{A}$. This changes the intersection number by at most 2 .

Denote the lift of $\kappa$ to $\Delta$ by $\tilde{\kappa}$. Clearly $\tilde{\kappa}$ contains at most two "corner arcs" (see Section 4) of $\tilde{\gamma} \cap \Delta$, which can be ignored in our count. If $p_{1} \leq q_{1}$ then $\tilde{\gamma}$ contains no horizontal strands and $i(\kappa, L) \leq 1$.

Now suppose $p_{1}>q_{1}$. In this case $\tilde{\gamma} \cap \Delta$ contains $m>0$ horizontal arcs running across $\tilde{A}$ joining $L$ to $S_{1}(L)$. Thus after entering $A$ across $\partial^{-} A$, the component $\kappa$ travels around $A$ cutting $\pi(L)$ either $m$ or $m+1$ times before exiting across $\partial^{+} A$. (This is the well known combinatorics of simple curves crossing a cylinder.) The total number of such connected components is $i\left(\sigma_{1}, \gamma\right)=q_{1}$. On the other hand, the total number of strands of $\tilde{\gamma} \cap \Delta$ which meet $L$ is by definition $p_{1}$. Thus $m q_{1} \leq p_{1}<$ $(m+1) q_{1}+q_{1}$ and so $m=\left[p_{1} / q_{1}\right]+O(1)$. Finally, we check from the definition that in the obvious metric $h_{0}$ on $\Delta$ in which $\widetilde{\sigma}_{1}$ is orthogonal to $\tilde{\gamma}_{T}$, we have $i_{\sigma_{1}}(\gamma, h)<0$ while $i_{\sigma_{1}}\left(\gamma_{T}, h\right)=0$. Thus $i_{\sigma_{i}}(\gamma, T)=-p_{i}(\gamma) / q_{i}(\gamma)+O(1)$ as claimed.

The arguments for $p_{1}<0$ and for $S_{2}$ are similar.

Proof of Proposition 6.14 From Lemma 6.18 we have $i_{\sigma_{i}}(\gamma, T)=-p_{i}(\gamma) / q_{i}(\gamma)+$ $O(1)$. On the other hand we can also compute $i_{\sigma_{i}}(\gamma, T)$ as follows. As usual, after normalizing suitably let $\widetilde{S}^{+}=\widetilde{S}_{i}^{+}$be the lift of $\sigma_{i}$ to $\partial \mathcal{C}^{+}$which is invariant under $S_{i}: z \mapsto z+2$. If $\tilde{\gamma}$ is a good lift of $\gamma$, then $\tilde{\gamma}$ certainly intersects $\widetilde{S}^{+}$. Now referring to Figure 3, let $B_{2}, B_{3}$ be the circles with equal diameters $2 / \Im \tau_{i}$ tangent to $\mathbb{R}$ at 0 , and $\mathbb{R}+\tau_{i}$ at $\tau_{i}$, respectively. It follows from the usual ping-pong theorem methods, that there is a lift $\widetilde{T}$ of the axis of $T$ to $\partial \mathcal{C}^{+}$which has one endpoint inside $B_{2}$ and one inside $B_{3}$. This lift also clearly cuts $\widetilde{S}^{+}$. By Lemma $6.17, i_{\sigma_{i}}(\gamma, T)$ is up to sign the number of images $S_{i}^{n}(\tilde{\gamma})$ of $\tilde{\gamma}$ which cut $\tilde{T}$. Since $\tilde{\gamma}$ is a good lift, orienting as in Lemma 6.17, we see that $i_{\sigma_{i}}(\gamma, T)=\left[\Re \tau_{i} / 2\right]+O(1)$. We deduce that

$$
\Re \tau_{i}=-2 p_{i}(\gamma) / q_{i}(\gamma)+O(1) \text {. }
$$

and the result follows.

Proof of Theorem $\mathbf{C}$ As usual let $\xi \in M L_{\mathbb{Q}}$ be admissible and let $G_{\xi}(\theta)$ be the unique group for which $\beta(G)=\theta \xi$. Let $h(\theta)$ denote the hyperbolic structure of $\partial \mathcal{C}^{+} / G_{\xi}(\theta)$. Since $l_{\sigma_{i}}^{+} \rightarrow 0, i=1,2$, the limit of the structures $h(\theta)$ in $P M L$ is in the linear span of $\delta_{\sigma_{1}}, \delta_{\sigma_{2}}$. We want to prove that the limit is the barycentre $\delta_{\sigma_{1}}+\delta_{\sigma_{2}}$. 
Let $\delta, \delta^{\prime} \in \mathcal{S}$. Since $\sigma_{1}, \sigma_{2}$ are a maximal set of simple curves on $\Sigma$, the thin part of $h(\theta)$ is contained in collars $A_{i}$ around $\sigma_{i}$ of approximate width $\log \left(1 / l_{\sigma_{i}}^{+}\right)$and the lengths of $\delta, \delta^{\prime}$ outside the collars $A_{i}$ are bounded (with a bound depending only on the combinatorics of $\delta, \delta^{\prime}$ and hence the canonical coordinates $\left.\mathbf{i}(\delta), \mathbf{i}\left(\delta^{\prime}\right)\right)$. By Proposition 6.14 the twisting around $A_{i}$ is bounded. We deduce that for any curve transverse to $\sigma_{i}$ we have

$$
l_{\delta}^{+}=\sum_{i=1,2} q_{i}(\delta) \log \left(1 / l_{\sigma_{i}}^{+}\right)+O(1)
$$

(see for example [23, Lemma 7.2]). By Theorem 6.1 we have $l_{\sigma_{1}}^{+} / l_{\sigma_{2}}^{+} \rightarrow q_{2}(\xi) / q_{1}(\xi)$, and since $\xi$ is admissible, $q_{1}(\xi), q_{2}(\xi)>0$. Thus $\log l_{\sigma_{1}}^{+} / \log l_{\sigma_{2}}^{+} \rightarrow 1$. Hence

$$
l_{\delta}^{+} / l_{\delta^{\prime}}^{+} \rightarrow \sum_{i=1,2} q_{i}(\delta) / \sum_{i=1,2} q_{i}\left(\delta^{\prime}\right)=i\left(\delta, \sigma_{1}+\sigma_{2}\right) / i\left(\delta^{\prime}, \sigma_{1}+\sigma_{2}\right) .
$$

The result follows from the definition of convergence to a point in $P M L$.

Remark 6.19 The above length estimate above coincides with that coming from the top terms Theorem 4.1. Namely from that formula we have

$$
\log \operatorname{Tr} \delta=\sum_{i=1,2} q_{i}(\delta) \log \left(\tau_{i}\right)+O(1) .
$$

Since $l_{\sigma_{i}}^{+}$is small, any transverse curve has definite length. Hence by for example Proposition 5.1 of [28], $l_{\delta}^{+}$is close to the hyperbolic length of the geodesic representative of $\delta$ in $\mathbb{H}^{3} / G$ and thus to $\log \operatorname{Tr} \delta$. Since by Proposition 6.6, $l_{\sigma_{i}}^{+} \Im \tau_{i}=O(1)$, the formula (11) is compatible with (10).

\section{Asymptotic directions}

In this section we prove our main results, Theorems A and B.

Throughout this section, to simplify notation, $X=O(\theta)$ will mean $X \leq c \theta$ where the constant $c$ depends on the lamination $\xi$ and a small number of related curves chosen during the proofs. With a bit more effort, the dependence could be controlled more carefully, but this is not needed for our results here. We write $\operatorname{Tr} \gamma$ to mean $\operatorname{Tr} \rho(W)$ where $W$ is a word representing $\gamma \in \pi_{1}(\mathcal{S})$.

Suppose that $\gamma$ is a bending line of the upper component $\partial \mathcal{C}^{+} / G$ of the convex hull boundary for a group $G\left(\tau_{1}, \tau_{2}\right)$ lying on a pleating ray $\mathcal{P}_{\xi}$. The top terms Theorem 4.1, together with the condition $\operatorname{Tr} \gamma \in \mathbb{R}$ of Lemma 3.3, gives asymptotic conditions 
for $\left(\tau_{1}, \tau_{2}\right) \in \mathcal{P}_{\xi}$, in terms of the canonical coordinates $\mathbf{i}(\gamma)$ of $\gamma$. For $\tau_{1}, \tau_{2} \in \mathbb{C}_{+}^{2}$ set $\tau_{i}=x_{i}+i y_{i}, \rho=\sqrt{y_{1}^{2}+y_{2}^{2}}$, and $\eta_{i}=y_{i} / \rho$. Define

$$
E_{\gamma}\left(\tau_{1}, \tau_{2}\right)=\left(q_{1} x_{1}+2 p_{1}\right) \eta_{2}+\left(q_{2} x_{2}+2 p_{2}\right) \eta_{1},
$$

where as usual $\mathbf{i}(\gamma)=\left(q_{1}, p_{1}, q_{2}, p_{2}\right)$ and $y_{i}>0, i=1,2$.

Proposition 7.1 Suppose that $\xi \in M L_{\mathbb{Q}}$ is an admissible lamination, that $G\left(\tau_{1}, \tau_{2}\right) \in$ $\mathcal{P}_{\xi}$ has bending measure $\beta(G)=\theta \xi$, and that $\gamma$ is a bending line of $\xi$. Then $E_{\gamma}\left(\tau_{1}, \tau_{2}\right)=O(\theta)$ as $\theta \rightarrow 0$.

Proof Suppose first that $q_{i}=q_{i}(\gamma)>0, i=1,2$ and set $a_{i}=-2 p_{i}(\gamma) / q_{i}(\gamma)$. By Theorem 4.1 we have

$$
\operatorname{Tr} \gamma= \pm 2^{\left|q_{2}-q_{1}\right|}\left(\tau_{1}-a_{1}\right)^{q_{1}}\left(\tau_{2}-a_{2}\right)^{q_{2}}+R\left(q_{1}+q_{2}-2\right)
$$

where $R\left(q_{1}+q_{2}-2\right)$ is a polynomial of degree at most $q_{i}$ in $\tau_{i}$ but with total degree in $\tau_{1}$ and $\tau_{2}$ at most $q_{1}+q_{2}-2$.

By Proposition 6.14, $x_{i}-a_{i}=O(1)$ and by Theorem 6.1

$$
\left|q_{2}(\xi) / q_{1}(\xi)-\eta_{1} / \eta_{2}\right|=O(\theta) .
$$

(Notice that the terms in (13) involve $q_{i}(\xi)$ as opposed to $q_{i}=q_{i}(\gamma)$ in (12).) Hence arranging the terms of (12) in order of decreasing powers of $\rho$, and using Equation (1) in Section 4, we get

$$
\begin{aligned}
\pm \operatorname{Tr} \gamma 2^{-\left|q_{2}-q_{1}\right|}=\rho^{q_{1}+q_{2}} \eta_{1}{ }^{q_{1}} \eta_{2}{ }^{q_{2}}+i \rho^{q_{1}+q_{2}-1} \eta_{1}{ }^{q_{1}-1} \eta_{2}{ }^{q_{2}-1}\left(q_{2} \eta_{1}\left(x_{2}-a_{2}\right)\right. \\
\left.+q_{1} \eta_{2}\left(x_{1}-a_{1}\right)\right)+O\left(\rho^{q_{1}+q_{2}-2}\right) .
\end{aligned}
$$

By Lemma 3.3, $\operatorname{Tr} \gamma \in \mathbb{R}$. We deduce $\eta_{1}{ }^{q_{1}-1} \eta_{2}{ }^{q_{2}-1} E_{\gamma}\left(\tau_{1}, \tau_{2}\right)+O(1 / \rho)=0$ from which using (13),

$$
\left.q_{2} \eta_{1}\left(x_{2}-a_{2}\right)+q_{1} \eta_{2}\left(x_{1}-a_{1}\right)\right)=O(1 / \rho) .
$$

Since $1 / \rho=O(\theta)$ by Corollary 6.5, this proves the result.

We still have to deal with the case that, say, $q_{2}(\gamma)=0$. Then $\operatorname{Tr} \gamma$ is a polynomial in $\tau_{1}$ only, of the form

$$
\operatorname{Tr} \rho(\gamma)= \pm 2^{q_{1}}\left(\tau_{1}-a_{1}\right)^{q_{1}}+R\left(q_{1}-2\right) .
$$

The result then follows easily by similar reasoning to the above. 


\subsection{Solving the asymptotic equations}

Suppose we want to locate the pleating ray $\mathcal{P}_{\gamma}$ of $\gamma \in S$. If $G \in \mathcal{P}_{\gamma}$, then $\partial \mathcal{C}^{+} / G \backslash \gamma$ is flat, so that not only $\gamma$, but also any curve $\delta \in \mathrm{wh}(\gamma)$, is a bending line of $\xi$, where $\operatorname{wh}(\gamma)$ (the wheel of $\gamma$ ) denotes the set of all curves $\delta \in \mathcal{S}$ disjoint from $\gamma$. (By convention, $\gamma \notin w h(\gamma)$.) Thus $\tau_{1}, \tau_{2}$ are constrained by the equations

$$
\Im \operatorname{Tr} \gamma=\Im \operatorname{Tr} \delta=0
$$

and hence, by the above proposition,

$$
E_{\gamma}\left(\tau_{1}, \tau_{2}\right)+O(\theta)=0 \quad \text { and } \quad E_{\delta}\left(\tau_{1}, \tau_{2}\right)+O(\theta)=0
$$

for all $\delta \in \mathrm{wh}(\gamma)$. Our proof of Theorem A amounts to solving these equations for $\tau_{1}, \tau_{2}$.

In order to do this, note that for any curve $\omega \in \mathcal{S}$,

$$
E_{\omega}\left(\tau_{1}, \tau_{2}\right)=\mathbf{i}(\omega) \cdot \mathbf{u}
$$

where $\mathbf{i}(\omega)=\left(q_{1}(\omega), p_{1}(\omega), q_{2}(\omega), p_{2}(\omega)\right)$ and

$$
\mathbf{u}=\left(x_{1} \eta_{2}, 2 \eta_{2}, x_{2} \eta_{1}, 2 \eta_{1}\right)
$$

with $x_{i}=\Re \tau_{i}, \eta_{i}=\Im \tau_{i} / \rho$ as above. Effectively what we will do is use linear algebra to solve the equations $\mathbf{i}(\delta) \cdot \mathbf{u}=0$ for all $\delta \in \mathrm{wh}(\gamma)$. This is done with the aid of Thurston's symplectic form $\Omega_{\mathrm{Th}}$ introduced in Section 4.2. This induces a map $\xi \rightarrow \xi^{*}$ of $\mathbb{R}^{4}$ such that

$$
\Omega_{\mathrm{Th}}(\mathbf{i}(\gamma), \mathbf{i}(\delta))=\mathbf{i}(\gamma) \cdot \mathbf{i}(\delta)^{*}
$$

where $\cdot$ is the usual inner product on $\mathbb{R}^{4}$ : if $\mathbf{i}(\gamma)=\left(q_{1}, p_{1}, q_{2}, p_{2}\right)$, then $\mathbf{i}(\gamma)^{*}=$ $\left(-p_{1}, q_{1},-p_{2}, q_{2}\right)$. By Proposition $4.3, \mathbf{i}(\gamma)^{*}$ is orthogonal not only to $\mathbf{i}(\gamma)$, but also to all curves in wh $(\gamma)$. We need:

\section{Lemma 7.2}

(i) Suppose that $\gamma, \delta \in \mathcal{S}$ and that $\delta \in \mathrm{wh}(\gamma)$. Then $\gamma$ and $\delta$ are supported on a common canonical train track and $\mathbf{i}(\gamma), \mathbf{i}(\delta)$ are independent vectors in $M L$.

(ii) Given $\gamma \in \mathcal{S}$, we can find $\delta, \delta^{\prime} \in \mathrm{wh}(\gamma)$ such that $\mathbf{i}(\gamma), \mathbf{i}(\delta), \mathbf{i}\left(\delta^{\prime}\right)$ are supported on a common canonical train track and span a subspace of dimension 3 in $M L$.

Proof (i) That $\gamma, \delta$ are supported on a common canonical train track follows immediately since they are disjoint. If $\mathbf{i}(\gamma), \mathbf{i}(\delta)$ are dependent, since they lie on the same track all their coefficients are integers which pairwise have the same sign. Thus we 
must have $n \mathbf{i}(\gamma)=m \mathbf{i}(\delta)$ for some $n, m \in \mathbb{N}$. Since both are connected simple curves, $n=m=1$.

(ii) The surface $\Sigma_{\gamma}:=\Sigma \backslash \gamma$ is either a one holed torus and a sphere with two punctures and a hole; or a sphere with two holes and two punctures. In either case, $\operatorname{PML}\left(\Sigma_{\gamma}\right)$ is a topological circle in which the set of rational laminations supported on a simple curve is dense. Let $\pi$ denote the map which associates to a curve $\omega \in \mathcal{S}$ the measured lamination $\delta_{\omega} \in M L(\Sigma)$, and likewise define the quotient map $\bar{\pi}(\omega)=\left[\delta_{\omega}\right] \in P M L$. Then $\pi, \bar{\pi}$ are injective and $\bar{\pi}(w h \gamma)$ is a dense subset of an embedded circle $K$ in $\operatorname{PML}(\Sigma)$.

If (ii) is false, then in particular $\pi(\mathrm{wh}(\gamma)) \subset M L$ is contained in a 2-plane whose image in $P M L$ is an affine line $L$. (Since the canonical coordinates are global coordinates for $M L$, there is no need to consider the subdivision of $M L$ into cells.) Since $P M L\left(\Sigma_{\gamma}\right)$ injects into $L$, it is open and closed in $K$. Thus $K=L$, which is impossible. Thus $\pi(w h(\gamma))$ spans at least a 3-dimensional subspace in $M L$.

Suppose that $\pi(\gamma)$ is in the linear span of $\pi(\delta), \pi\left(\delta^{\prime}\right) \in \pi(\operatorname{wh}(\gamma)) \subset M L$. Then by the above we can find $\delta^{\prime \prime} \in \operatorname{wh}(\gamma)$ with $\pi\left(\delta^{\prime \prime}\right)$ not contained in the linear span of $\pi(\gamma), \pi(\delta)$ and $\pi\left(\delta^{\prime}\right)$ which proves the result.

Theorem 7.3 (Theorem A) Suppose that $\xi=\sum_{1,2} a_{i} \gamma_{i}$ is admissible and not exceptional. Let $\mathbf{i}(\xi)=\left(q_{1}, p_{2}, q_{2}, p_{2}\right)$ and set $\tan \psi(\xi)=q_{1} / q_{2}$. Let $L_{\xi}:[0, \infty) \rightarrow \mathbb{C}^{2}$ be the line $t \mapsto\left(w_{1}(t), w_{2}(t)\right)$ where

$$
w_{1}(t)=-2 p_{1} / q_{1}+t \cos \psi, w_{2}(t)=-2 p_{2} / q_{2}+t \sin \psi .
$$

Let $\left(\tau_{1}(\theta), \tau_{2}(\theta)\right) \in \mathbb{C}^{2}$ be the point corresponding to the group $G_{\xi}(\theta)$ with $\beta(G)=\theta \xi$, so that the pleating ray $\mathcal{P}_{\xi}$ is the image of the map $p_{\xi}: \theta \rightarrow\left(\tau_{1}(\theta), \tau_{2}(\theta)\right)$ for a suitable range of $\theta>0$. Then $\mathcal{P}_{\xi}$ approaches $L_{\xi}$ as $\theta \rightarrow 0$ in the sense that if $t(\theta)=4 Q / \theta q_{1} q_{2}$ with $Q=\sqrt{q_{1}^{2}+q_{2}^{2}}$ then

$$
\left|\Re \tau_{i}(\theta)-\Re w_{i}(t(\theta))\right|=O(\theta) \quad \text { and } \quad\left|\Im \tau_{i}(\theta)-\Im w_{i}(t(\theta))\right|=O(1), \quad i=1,2 .
$$

Proof As above, write $\tau_{i}(\theta)=\tau_{i}=x_{i}+i y_{i}, \rho^{2}=y_{1}^{2}+y_{2}^{2}$ and $\eta_{i}=y_{i} / \rho$, where the dependence on $\theta$ is understood. By Theorem 6.1, we have $y_{i}-4 / \theta q_{i}=O(1)$.

On the other hand, with $t=t(\theta)$ as in the statement of the theorem, we find $\Im w_{1}(t)=$ $t q_{2} / Q=4 / \theta q_{1}$ and similarly $\Im w_{2}(t)=4 / \theta q_{2}$. Thus $\left|\Im \tau_{i}(\theta)-\Im w_{i}(t(\theta))\right|=$ $O(1), i=1,2$ as $\theta \rightarrow 0$.

To deal with the coordinates $x_{i}=\Re \tau_{i}(\theta)$ is more subtle. Consider first the special case $\xi=\gamma \in \mathcal{S}$. By Lemma 7.2 we can choose $\delta, \delta^{\prime} \in \mathrm{wh}(\gamma)$ such that $\mathbf{i}(\gamma), \mathbf{i}(\delta), \mathbf{i}\left(\delta^{\prime}\right)$ 
span a subspace of dimension 3 in $M L$. If $\left(\tau_{1}, \tau_{2}\right) \in \mathcal{P}_{\gamma}$, the conditions

$$
\mathfrak{\Im} \operatorname{Tr} \gamma=\mathfrak{s} \operatorname{Tr} \delta=\Im \operatorname{Tr} \delta^{\prime}=0
$$

must be satisfied. By Proposition 7.1, we can write this as

$$
E_{\zeta}\left(\tau_{1}, \tau_{2}\right)+O(\theta)=0
$$

for $\zeta=\gamma, \delta, \delta^{\prime}$. With $\tau_{i}=x_{i}+i \rho \eta_{i}$ as above, we can as in (15) regard these as equations in $\mathbb{R}^{4}$ for $\mathbf{u}=\mathbf{u}(\theta)=\left(x_{1} \eta_{2}, 2 \eta_{2}, x_{2} \eta_{1}, 2 \eta_{1}\right)$,

$$
\mathbf{i}(\zeta) \cdot \mathbf{u}=O(\theta)
$$

for $\zeta=\gamma, \delta, \delta^{\prime}$. Now we use Thurston's symplectic form. By Proposition 4.3 we have $\Omega_{\mathrm{Th}}(\mathbf{i}(\gamma), \mathbf{i}(\zeta))=0$ for all $\zeta \in \operatorname{wh}(\gamma)$. Hence

$$
\mathbf{i}(\zeta) \cdot \mathbf{i}(\gamma)^{*}=0
$$

for $\zeta=\gamma, \delta, \delta^{\prime}$. Since $\mathbf{i}(\gamma), \mathbf{i}(\delta), \mathbf{i}\left(\delta^{\prime}\right)$ are independent, it follows that we can write

$$
\mathbf{u}(\theta)=\lambda(\theta) \mathbf{i}(\gamma)^{*}+\mu(\theta) \mathbf{v}(\theta)
$$

where $\mathbf{v}=\mathbf{v}(\theta)$ is in the linear span of $\mathbf{i}(\gamma), \mathbf{i}(\delta), \mathbf{i}\left(\delta^{\prime}\right)$ and $\|\mathbf{v}\|=1$. We find using (16) that $\mathbf{u} \cdot \mathbf{v}=O(\theta)$ (where the constants depend on $\left.\mathbf{i}(\gamma), \mathbf{i}(\delta), \mathbf{i}\left(\delta^{\prime}\right)\right)$. Then $\mathbf{v} \cdot \mathbf{i}\left(\gamma^{*}\right)=0$ gives $\mu(\theta)=O(\theta)$, with the same proviso on the constants. Equating the two sides of (17) gives

$$
\begin{array}{ll}
x_{1} \eta_{2}=-\lambda p_{1}(\gamma)+O(\theta), & 2 \eta_{2}=\lambda q_{1}(\gamma)+O(\theta), \\
x_{2} \eta_{1}=-\lambda p_{2}(\gamma)+O(\theta), & 2 \eta_{1}=\lambda q_{2}(\gamma)+O(\theta) .
\end{array}
$$

Since $\|\mathbf{u}\|^{2}=\left(x_{1}^{2}+4\right) \eta_{2}^{2}+\left(x_{2}^{2}+4\right) \eta_{1}^{2} \geq 4$ we find

$$
|\lambda(\theta)|=\frac{\|\mathbf{u}(\theta)-O(\theta)\|}{\left\|\mathbf{i}(\gamma)^{*}\right\|} \geq c
$$

for some constant $c>0$. It follows easily that $\left|x_{i}+2 p_{i} / q_{i}\right|=O(\theta)$, proving Theorem A in the special case $\xi=\gamma$.

Now we turn to the case of a general admissible lamination $\xi=a_{\gamma} \gamma+a_{\delta} \delta \in M L_{\mathbb{Q}}$. In this case, if $\left(\tau_{1}, \tau_{2}\right) \in \mathcal{P}_{\xi}$ then $\gamma$ and $\delta$ are both bending lines of $G\left(\tau_{1}, \tau_{2}\right)$. It follows as above that

$$
\mathbf{i}(\gamma) \cdot \mathbf{u}=O(\theta) \quad \text { and } \quad \mathbf{i}(\delta) \cdot \mathbf{u}=O(\theta) .
$$

By Lemma 7.2, $\mathbf{i}(\gamma)$ and $\mathbf{i}(\delta)$ are independent and by Proposition 4.3, $\mathbf{i}(\gamma)^{*}$ and $\mathbf{i}(\delta)^{*}$ are orthogonal to both. Thus

$$
\mathbf{u}=\lambda \mathbf{i}(\gamma)^{*}+\mu \mathbf{i}(\delta)^{*}+\nu \mathbf{w}
$$


where $\mathbf{w}$ is in the span of $\mathbf{i}(\gamma), \mathbf{i}(\delta)$, and $\|\mathbf{w}\|=1$. Thus $\mathbf{u} \cdot \mathbf{w}=O(\theta)$ and since $\mathbf{w} \cdot \mathbf{i}(\gamma)^{*}=\mathbf{w} \cdot \mathbf{i}(\delta)^{*}=0$ we find $v=v\|\mathbf{w}\|=\mathbf{u} \cdot \mathbf{w}=O(\theta)$.

Now from Theorem 6.1,

$$
\left|\frac{y_{2}}{y_{1}}-\frac{a_{\gamma} q_{1}(\gamma)+a_{\delta} q_{1}(\delta)}{a_{\gamma} q_{2}(\gamma)+a_{\delta} q_{2}(\delta)}\right|=O(\theta) .
$$

On the other hand setting $z_{i}=\lambda q_{i}(\gamma)+\mu q_{i}(\delta)$, we find from (19) that $\left|z_{1}\right|^{2}+\left|z_{2}\right|^{2} \geq$ $c>0$ for some constant $c$ depending only on $\gamma, \delta$. It follows from (19) and (20) that $\left|z_{2} / z_{1}-y_{2} / y_{1}\right|=O(\theta)$. Since by hypothesis we are not in the exceptional case $q_{1}(\gamma) / q_{2}(\gamma)=q_{1}(\delta) / q_{2}(\delta)$, we deduce that $\left|\lambda / \mu-a_{\gamma} / a_{\delta}\right|=O(\theta)$. Substituting back in (19) we see $\mathbf{u}=\kappa i(\xi)^{*}+O(\theta)$ for some $\kappa>0$, and a little bit of algebra completes the proof.

The following lemma proves the second part of the statement of Theorem B.

Lemma 7.4 Suppose that $\gamma_{1} \in \mathcal{S}$ is admissible. Then there exists $\gamma_{2} \in \mathrm{wh}\left(\gamma_{1}\right)$ such that the pair $\gamma_{1}, \gamma_{2}$ is not exceptional.

Proof Write $\mathbf{X}=\left(X_{1}, X_{2}, X_{3}, X_{4}\right) \in \mathbb{R}^{4}$. Then $\delta \in \mathrm{wh}\left(\gamma_{1}\right)$ implies that $\mathbf{i}(\delta)$ is in the codimension one hyperplane $H$ defined by $\mathbf{i}\left(\gamma_{1}\right)^{*} \cdot \mathbf{X}=0$. If the pair $\gamma_{1}, \delta$ is exceptional then $\mathbf{i}(\delta)$ is also in the codimension one hyperplane $K$ defined by $q_{1}(\gamma) X_{1}-q_{2}(\gamma) X_{3}=0$. Note that $\mathbf{i}(\gamma)$ is also in $H \cap K$.

Now since $q_{1}(\gamma), q_{2}(\gamma)>0$, the normal vectors

$$
\left(-p_{1}(\gamma), q_{1}(\gamma),-p_{2}(\gamma), q_{2}(\gamma)\right) \quad \text { and } \quad\left(q_{1}(\gamma), 0, q_{2}(\gamma), 0\right)
$$

to $H$ and $K$ respectively are not collinear. So $H \cap K$ is two dimensional. However by Lemma 7.2, we can find $\delta, \delta^{\prime} \in \mathrm{wh}\left(\gamma_{1}\right)$ such that $\mathbf{i}(\delta), \mathbf{i}\left(\delta^{\prime}\right), \mathbf{i}\left(\gamma_{1}\right)$ are independent, so at least one of the pairs $\mathbf{i}\left(\gamma_{1}\right), \mathbf{i}(\delta)$ and $\mathbf{i}\left(\gamma_{1}\right), \mathbf{i}\left(\delta^{\prime}\right)$ must be nonexceptional as claimed.

Proof of Theorem B We need to show that if $\xi=\sum_{1,2} a_{i} \delta_{\gamma_{i}}$ is admissible and if the pair $\gamma_{1}, \gamma_{2}$ is not exceptional, then the equations

$$
\operatorname{Tr} \gamma_{1}, \operatorname{Tr} \gamma_{2} \in \mathbb{R}
$$

have a unique solution as $\tau_{i} \rightarrow \infty$ in the direction specified by Theorem A. First consider the equations

$$
\tau_{1}^{q_{1}} \tau_{2}^{q_{2}}, \tau_{1}^{q_{1}^{\prime}} \tau_{2}^{q_{2}^{\prime}} \in \mathbb{R},
$$


as $\tau_{1}, \tau_{2} \rightarrow \infty$, where $q_{i}=q_{i}\left(\gamma_{1}\right)$ and $q_{i}^{\prime}=q_{i}\left(\gamma_{2}\right)$. Setting $z_{i}=1 / \tau_{i}$ we define $G: \mathbb{C}^{2} \rightarrow \mathbb{C}^{2}$ by

$$
G\left(z_{1}, z_{2}\right)=\left(z_{1}^{q_{1}} z_{2}^{q_{2}}, z_{1}^{q_{1}^{\prime}} z_{2}^{q_{2}^{\prime}}\right),
$$

so that (21) is equivalent to the equation $G\left(z_{1}, z_{2}\right) \in \mathbb{R}^{2}$ as $z_{1}, z_{2} \rightarrow 0$. Writing $z_{i}=\epsilon_{i} e^{i \theta_{i}}, i=1,2$ we obtain

$$
e^{i\left(q_{1} \theta_{1}+q_{2} \theta_{2}\right)}, e^{i\left(q_{1}^{\prime} \theta_{1}+q_{2}^{\prime} \theta_{2}\right)} \in \mathbb{R},
$$

so that writing

$$
A=\left(\begin{array}{ll}
q_{1} & q_{2} \\
q_{1}^{\prime} & q_{2}^{\prime}
\end{array}\right)
$$

we must have $A\left(\theta_{1}, \theta_{2}\right)^{T}=\pi(n, m)^{T}$ for $n, m \in \mathbb{Z}$. Since $A$ is invertible by hypothesis, these equations have a discrete set of solutions for $\left(\theta_{1}, \theta_{2}\right)$. Since $q_{1}+q_{2}, q_{1}^{\prime}+q_{2}^{\prime} \in 2 \mathbb{Z}$, one of these solutions is $\theta_{i}=\pi / 2, i=1,2$. It follows that $G$ is a branched covering $\mathbb{C}^{2} \rightarrow \mathbb{C}^{2}$ in a neighbourhood of 0 , and $G^{-1}\left(\mathbb{R}^{2}\right)$ is a discrete set of 2-planes meeting only at 0 .

Now consider our actual equations $\Im \operatorname{Tr} \gamma=\mathfrak{\Im} \operatorname{Tr} \delta=0$. As above set $z_{i}=1 / \tau_{i}$ and define $H: \mathbb{C}^{2} \rightarrow \mathbb{C}^{2}$ by $H\left(z_{1}, z_{2}\right)=(1 / \operatorname{Tr} \gamma, 1 / \operatorname{Tr} \delta)$. By Theorem 4.1 we have

$$
\begin{aligned}
& \operatorname{Tr} \gamma=\left(\tau_{1}+2 p_{1} / q_{1}\right)^{q_{1}}\left(\tau_{2}+2 p_{2} / q_{2}\right)^{q_{2}}\left(1+R_{1}\right), \\
& \operatorname{Tr} \delta=\left(\tau_{1}+2 p_{1}^{\prime} / q_{1}^{\prime}\right)^{q_{1}^{\prime}}\left(\tau_{2}+2 p_{2}^{\prime} / q_{2}^{\prime}\right)^{q_{2}^{\prime}}\left(1+R_{2}\right) .
\end{aligned}
$$

where $p_{i}=p_{i}(\gamma), q_{i}=q_{i}(\gamma)$ and $p_{i}^{\prime}=p_{i}(\delta), q_{i}^{\prime}=q_{i}(\delta)$ and $R_{1}, R_{2}$ denote polynomials of total order at most $q_{1}+q_{2}-2$ in $\tau_{1}, \tau_{2}$. Hence we can expand $H\left(z_{1}, z_{2}\right)$ as a Taylor expansion about 0 to obtain

$$
H\left(z_{1}, z_{2}\right)=\left(z_{1}^{q_{1}} z_{2}^{q_{2}}\left(1+p_{1} z_{1}+p_{2} z_{2}+\widehat{R}_{1}\right), z_{1}^{q_{1}^{\prime}} z_{2}^{q_{2}^{\prime}}\left(1+p_{1}^{\prime} z_{1}+p_{2}^{\prime} z_{2}+\widehat{R}_{2}\right)\right)
$$

where $\hat{R}_{1}, \hat{R}_{2}$ denote terms of total order at least 2 in $z_{1}, z_{2}$ and $p_{i}=p_{i}(\gamma)$, $p_{i}^{\prime}=p_{i}(\delta)$.

There is clearly a neighbourhood $U$ of 0 such that $H$ is locally injective on $U \backslash\{0\}$, and moreover in which the homotopy $G+t H, t \in[0,1]$ between $G$ and $H$ is regular at every point. It follows that $H$ is also a branched covering of $\mathbb{C}^{2}$ near zero, of the same order as $G$, and that there is a natural bijective correspondence between the branches of $G^{-1}\left(\mathbb{R}^{2}\right)$ and $H^{-1}\left(\mathbb{R}^{2}\right)$. To complete the proof, we need to show that for $\left(t_{1}, t_{2}\right) \in \mathbb{R}^{2}$ sufficiently near 0 , the point $H^{-1}\left(t_{1}, t_{2}\right)$ is arbitrarily close to the point $G^{-1}\left(t_{1}, t_{2}\right)$ on the corresponding sheet of $G^{-1}\left(\mathbb{R}^{2}\right)$.

In a neighbourhood of 0 , we view (24) as a perturbation of (21) and use the ideas of Appendix B in [22]. If $g: \mathbb{C}^{n} \rightarrow \mathbb{C}^{n}$ is an analytic function with an isolated zero at 
$Z_{0} \in \mathbb{C}^{n}$, define the multiplicity of $g$ to be the degree of the mapping $g /\|g\|: S_{\delta} \rightarrow S_{1}$, where $S_{\delta}$ is the sphere radius $\delta$ centre $Z_{0}$ and $S_{1}$ is the unit sphere. The same proof as Lemma B.1 of [22] proves "Rouché's principle" that if $r: \mathbb{C}^{n} \rightarrow \mathbb{C}^{n}$ with $r(0)=0$ and if $\|r\|<\|g\|$ on $S_{\delta}$, then the degrees of $(g+r) /\|g+r\|$ and $g /\|g\|$ on $S_{\delta}$ are equal.

Now take $Z_{0} \in U$ to be an isolated solution of the equation $G\left(z_{1}, z_{2}\right)=\left(t_{1}, t_{2}\right) \in \mathbb{R}^{2}$. Choose $\delta>0$ so that so that $S_{\delta}\left(Z_{0}\right) \subset U$ and so that $G\left(z_{1}, z_{2}\right)=\left(t_{1}, t_{2}\right)$ has no other solutions in $S_{\delta}\left(Z_{0}\right)$. We can also choose $U$ small enough so that $\|(H-G)\|<\|G\|$ on $U$. It follows from the above Rouché's principle that $H$ and $G$ have the same degree on $S_{\delta}$. Then Lemma B.2 of [22] shows that $H$ has exactly one zero inside $S_{\delta}$ as required.

In particular, there is a unique branch of $H^{-1}\left(\mathbb{R}^{2}\right)$ close to the branch $z_{i}=\epsilon_{i} i, i=1,2$ of $G\left(z_{1}, z_{2}\right) \in \mathbb{R}^{2}$. If $\epsilon_{1} / \epsilon_{2}$ is bounded away from 0 and $\infty$ and we set $\epsilon=\sqrt{\epsilon_{1}^{2}+\epsilon_{2}^{2}}$, we can clearly write points on this branch in the form $z_{i}=\epsilon_{i} i e^{i \alpha_{i}}, i=1,2$ where $\alpha_{i}=O(\epsilon)$ as $\epsilon \rightarrow 0$. The arguments of Theorem A are then sufficient to show the solution to the equations in that theorem is unique.

\subsection{The exceptional case}

Recall a pair of curves $\gamma_{1}, \gamma_{2}$ is said to be exceptional if $q_{1}\left(\gamma_{1}\right) q_{2}\left(\gamma_{2}\right)=q_{1}\left(\gamma_{2}\right) q_{2}\left(\gamma_{1}\right)$. As an example, the coordinates $\mathbf{i}\left(\gamma_{1}\right)=(2,0,2,1)$ and $\mathbf{i}\left(\gamma_{2}\right)=(2,-1,2,2)$, or more generally $\mathbf{i}\left(\gamma_{1}\right)=(a+b, 0, a+b, a)$ and $\mathbf{i}\left(\gamma_{2}\right)=(a+b,-1, a+b, a+1)$, can be easily be checked to represent exceptional pairs of disjoint connected curves.

Theorem 7.5 Suppose that $\xi=\sum_{1,2} a_{i} \gamma_{i}$ is an admissible lamination such that the pair $\gamma_{1}, \gamma_{2}$ is exceptional. For $s \in[0,1]$, let $\xi(s)=\sum_{1,2} s a_{1} \gamma_{1}+(1-s) a_{2} \gamma_{2}$. Let $\mathbf{i}(\xi(s))=\left(q_{1}(s), p_{2}(s), q_{2}(s), p_{2}(s)\right)$ and set $\tan \psi(s)$ to equal $q_{1}(s) / q_{2}(s)$. Let $L_{\gamma_{1}, \gamma_{2}}:[0,1] \times[0, \infty) \rightarrow \mathbb{C}^{2}$ be the map $(s, t) \mapsto\left(w_{1}(s, t), w_{2}(s, t)\right)$ where

$$
w_{1}(s, t)=2 p_{1}(s) / q_{1}(s)+t \cos \psi(s), \quad w_{2}(s, t)=2 p_{2}(s) / q_{2}(s)+t \sin \psi(s) .
$$

Let $\left(\tau_{1}(s, \theta), \tau_{2}(s, \theta)\right) \in \mathbb{C}^{2}$ be the point corresponding to the group $G_{\xi(s)}(\theta)$ with $\beta(G)=\theta \xi(s)$, so that the (closure of the) pleating plane $\mathcal{P}_{\gamma_{1}, \gamma_{2}}$ is the image of the map $p_{\gamma_{1}, \gamma_{2}}:(s, \theta) \rightarrow\left(\tau_{1}(\theta), \tau_{2}(\theta)\right)$ for $s \in[0,1]$ and a suitable range of $\theta>0$. Then $\mathcal{P}_{\gamma_{1}, \gamma_{2}}$ approaches $L_{\gamma_{1}, \gamma_{2}}$ as $\theta \rightarrow 0$ in the sense that, letting $Q=\sqrt{\left(q_{1}^{2}(s)+q_{2}^{2}(s)\right)}$, if $t(s, \theta)=4 Q / \theta q_{1}(s) q_{2}(s)$, then for all sufficiently small $\theta$ there exists a continuous function $f_{\theta}:[0,1] \rightarrow[0,1]$ such that $f_{\theta}(0)=0, f_{\theta}(1)=1$ and

$$
\begin{aligned}
\left|\Re \tau_{i}(s, \theta)-\Re w_{i}\left(f_{\theta}(s), t(s, \theta)\right)\right| & =O(\theta), \\
\left|\Im \tau_{i}(s, \theta)-\Im w_{i}\left(f_{\theta}(s), t(s, \theta)\right)\right| & =O(1),
\end{aligned}
$$


for $i=1,2$. Moreover every point on $L_{\gamma_{1}, \gamma_{2}}$ is close to a point on $\mathcal{P}_{\gamma_{1}, \gamma_{2}}$, in the sense that for all sufficiently small $\theta$, for each $s$ there exists $s^{\prime} \in[0,1]$ such that

$$
\begin{aligned}
\left|\Re \tau_{i}\left(s^{\prime}, \theta\right)-\Re w_{i}\left(s, t\left(s^{\prime}, \theta\right)\right)\right| & =O(\theta), \\
\left|\Im \tau_{i}\left(s^{\prime}, \theta\right)-\Im w_{i}\left(s, t\left(s^{\prime}, \theta\right)\right)\right| & =O(1) .
\end{aligned}
$$

Remark 7.6 The difference between this statement and that of Theorem 7.3 is that in that theorem, $\mathcal{P}_{\xi(s)}$ is close to a point on $L_{\xi(s)}$, while here we can only assert closeness of points on the pleating ray $\mathcal{P}_{\xi(s)}$ to a point on some line $L_{\xi\left(f_{\theta}(s)\right)}$ where possibly $f_{\theta}(s) \neq s$.

Proof We proceed as in the proof of Theorem 7.3 above, up to (19) which says that $\mathbf{u}=\mathbf{u}(\theta)$ is close to a convex combination of $\mathbf{i}\left(\gamma_{1}\right)^{*}$ and $\mathbf{i}\left(\gamma_{2}\right)^{*}$. Define $f_{\theta(s)}$ by letting $\xi\left(f_{\theta(s)}\right)$ be the orthogonal projection of $\mathbf{u}(s, \theta)$ onto the plane spanned by $\mathbf{i}(\gamma)^{*}, \mathbf{i}(\delta)^{*}$. Then (25) follows as before, while continuity of the path $s \mapsto f_{\theta}(s)$ is clear. Equation (26) follows choosing $s^{\prime}$ such that $s=f_{\theta}\left(s^{\prime}\right)$.

Remark 7.7 One might expect to be able to prove Theorem A in the exceptional case by a limiting argument with laminations $\xi_{n} \rightarrow \xi$. However the interaction of the double limits as $n \rightarrow \infty$ and $\theta \rightarrow 0$ is quite subtle and we have not been able to extract the required results by this method.

Remark 7.8 Suppose that the pair $\gamma_{1}, \gamma_{2}$ is exceptional. Then we claim that it is not possible to deduce from the top terms Theorem 4.1 alone that the equations $\Im \operatorname{Tr} \gamma_{i}=0, i=1,2$ have a unique branch at infinity satisfying the conditions $\arg \tau_{i}=$ $\pi / 2, \mathfrak{s} \tau_{1} / \mathfrak{\Im} \tau_{2}=q_{2}(\xi) / q_{1}(\xi)$. To study this question, as above we replace the equations by equations $\mathfrak{I} f_{1}\left(z_{1}, z_{2}\right)=\mathfrak{I} f_{2}\left(z_{1}, z_{2}\right)=0$ in a neighbourhood of 0 , where $f_{i}: \mathbb{C}^{2} \rightarrow$ $\mathbb{C}^{2}$ are analytic functions with lowest order terms $z_{1}^{q_{1}\left(\gamma_{1}\right)} z_{2}^{q_{2}\left(\gamma_{1}\right)}$ and $z_{1}^{q_{1}\left(\gamma_{2}\right)} z_{2}^{q_{2}\left(\gamma_{2}\right)}$ respectively.

To show that many different behaviours are possible, consider the functions $f_{0}\left(z_{1}, z_{2}\right)=$ $z_{1} z_{2}, f_{1}\left(z_{1}, z_{2}\right)=z_{1} z_{2}\left(1-z_{1}+z_{2}+z_{1} z_{2}\right), f_{2}\left(z_{1}, z_{2}\right)=z_{1} z_{2}\left(1-z_{1}-z_{2}+z_{1} z_{2}\right)$ and $f_{3}\left(z_{1}, z_{2}\right)=z_{1} z_{2}\left(1-z_{1}+z_{2}+z_{1}^{2}\right)$. We look for solutions to each of the three pairs of equations $\Im f_{0}=0, \Im f_{i}=0, i=1,2,3$ which satisfy $z_{1}=\epsilon i e^{i \alpha}, z_{2}=\epsilon i e^{-i \alpha}$ where $\epsilon \rightarrow 0$ and $\alpha=O(\epsilon)$.

As is easily verified, the equations $\mathfrak{s} f_{0}=0, \mathfrak{s} f_{1}=0$ are satisfied for arbitrary choices of $\alpha$. The equations $\mathfrak{s} f_{0}=0, \mathfrak{s} f_{2}=0$ have no solutions of the required form near $(0,0)$. Finally the equations $\mathfrak{s} f_{0}=0, \mathfrak{s} f_{3}=0$ have a unique suitable solution for each $\epsilon>0$, namely $\alpha=0$. 


\section{Appendix 1}

The following proofs are taken from [13].

Proof of Proposition 2.1 Let $S_{3}=T S_{2} T^{-1}$, so that $S_{3}$ is parabolic with fixed point $T(0)=\tau_{1}$. Let $J_{j}=\left\langle S_{j}\right\rangle$ for $j=1,2,3$. We construct fundamental domains $D_{j}$ for the $S_{j}$ as follows.

Referring to Figure 3 in Section 2.2, let $l$ consist of the vertical line below $-1+i / 2$, the vertical line above $\tau_{1}-1-i / 2$ and the straight line joining $-1+i / 2$ to $\tau_{1}-1-i / 2$. This last line segment has positive slope because $\Im \tau_{1}>1$, from which it follows that $l$ and $S_{1}(l)$ do not intersect and hence that the strip $D_{1}$ between $l$ and $S_{1}(l)$ is a fundamental domain for $J_{1}$. Fundamental domains for $J_{2}$ and $J_{3}$ are

$$
\begin{aligned}
& D_{2}=\{z \in \widehat{\mathbb{C}}:|z+1 / 2|>1 / 2,|z-1 / 2|>1 / 2\}, \\
& D_{3}=\left\{z \in \widehat{\mathbb{C}}:\left|z-\tau_{1}+1 / 2\right|>1 / 2,\left|z-\tau_{1}-1 / 2\right|>1 / 2\right\} .
\end{aligned}
$$

The hypothesis $\Im \tau_{1}>1$ implies that the union of the closure of any two of the $D_{j}$ is the whole of $\widehat{\mathbb{C}}$. Moreover the boundaries of the $D_{j}$ only intersect at parabolic fixed points. Therefore by a simple application of the first Klein-Maskit combination theorem (see Maskit [20, page 149] or Beardon [3, page 103]), we see that $F_{0}=\left\langle S_{1}, S_{2}, S_{3}\right\rangle$ is discrete with fundamental domain $D_{0}=D_{1} \cap D_{2} \cap D_{3}$.

Now let $J_{4}=\langle T\rangle$. We will construct a fundamental domain $D_{4}$ for $J_{4}$. Let $B_{2}$ be the disk centred at $i / \mathfrak{\Im} \tau_{2}$ with radius $1 / \mathfrak{\Im} \tau_{2}$ and let $B_{3}$ be the disk centred at $\tau_{1}-i / \Im \tau_{2}$ with radius $1 / \Im \tau_{2}$. One checks that $T$ takes $B_{2}$ to the complement of $B_{3}$. (Note that $T(z)=\tau_{1}+1 /\left(\tau_{2}+1 / z\right)$ and consider the action of $z \mapsto 1 /\left(\tau_{2}+1 / z\right)$ on $\left.B_{2}.\right)$ Thus the domain $D_{4}$ exterior to both disks is a fundamental domain for $J_{4}$. Since $\Im \tau_{2}>1$ and $\Im \tau_{1} \Im \tau_{2}>4$, it is easy to see that $B_{2}$ and $B_{3}$ are contained in the strip $D_{1}, B_{2}$ is contained in $D_{3}$ and $B_{3}$ is contained in $D_{2}$. Moreover $S_{j}\left(B_{j}\right)=B_{j}$ for $j=2,3$. Thus $B_{j}$ is precisely invariant with respect to $J_{j}$ for $j=2,3$; that is, $W\left(B_{j}\right)=B_{j}$ for $W \in J_{j}$ and $W\left(B_{j}\right) \cap B_{j}=\varnothing$ for $W \in F_{0}-J_{j}$. Therefore the hypotheses of the second combination theorem are satisfied and so $G_{0}=\left\langle F_{0}, T\right\rangle$ is discrete with domain $D=D_{1} \cap D_{2} \cap D_{3} \cap D_{4}$.

Now we verify that $G \in \mathcal{M}$. By construction, $D$ consists of three components. The first, in the lower half plane $\mathbb{H}^{-}$, is a component of a fundamental domain for the Fuchsian subgroup $F_{2}=\left\langle S_{1}, S_{2}\right\rangle$ and $\mathbb{H}^{-} / F_{2}$ is a triply punctured sphere. Similarly the second, in the half plane $\mathbb{H}^{\tau_{1}}$ above the horizontal through $\tau_{1}$, is a component of a fundamental domain for the Fuchsian subgroup $F_{3}=\left\langle S_{1}, S_{3}\right\rangle$. Again $\mathbb{H}^{\tau_{1}} / F_{3}$ is a triply punctured sphere. Because the components of $D$ in $\mathbb{H}^{-}$and $\mathbb{H}^{\tau_{1}}$ are disjoint, $F_{2}$ and $F_{3}$ are not conjugate in $G$. 
Let $D^{*}$ denote the third component $D$. It is contained in the strip between the horizontal lines through 0 and $\tau_{1}$. It has eight sides, one pair of sides contained in the boundaries of each of $D_{1}, D_{2}, D_{3}, D_{4}$ and identified by $S_{1}, S_{2}, S_{3}$ and $T$ respectively. Performing these identifications we obtain a torus with two punctures corresponding to $S_{2} S_{1}^{-1}$ and $S_{3} S_{1}^{-1}$. Developing $D^{*}$ by $G$ we see that it corresponds to a simply connected $G$-invariant component of the regular set of $G$, and we conclude that $G \in \mathcal{M}$.

Finally, since translates of $D$ by $S_{1}$ cover the strip $1 / 2<\Im z<\Im \tau_{1}-1 / 2$, and since $\mathbb{H}^{-}, \mathbb{H}^{\tau_{1}}$ are in $\Omega(G)$, the limit set $\Lambda$ is contained in the two strips $0 \leq \Im z \leq 1 / 2$ and $\Im \tau_{1}-1 / 2 \leq \Im z \leq \Im \tau_{1}$ as claimed.

Proof of Proposition 2.2 This is based on a similar result for the Maskit space of the once punctured torus due to David Wright [30]. Let $W \in G-\left\{\left\langle S_{1}, S_{2}\right\rangle \cup\left\langle S_{1}, S_{3}\right\rangle\right\}$ and let $\mathbb{H}^{-}$be the lower half plane. Then $W\left(\mathbb{H}^{-}\right)$is a disk contained in the strip $\left\{0<\Im z<\Im \tau_{1}\right\}$. Let

$$
W=\left(\begin{array}{ll}
a & b \\
c & d
\end{array}\right)
$$

with $a d-b c=1$ and suppose that the circle $C=W(\mathbb{R} \cup \infty)$ has radius $r$ and centre $z_{0}$, so that $\Im z_{0} \geq r>0$. Using the fact that the points $T^{-1}\left(z_{0}\right), T^{-1}(\infty)$ are inverse points with respect to $\mathbb{R}$ (see also Mumford, Series and Wright [25, page 91]), we find that $r=i /(c \bar{d}-d \bar{c})$ and $z_{0}=(a \bar{d}-b \bar{c}) /(c \bar{d}-d \bar{c})$. The inequality $\Im z_{0}>0$ gives $\Im c \bar{d}>0$ and then $\Im z_{0} \geq r$ simplifies to $\Re(b \bar{c}-a \bar{d}) \geq 1$.

Applying this to $T$ we see that $\mathfrak{\Im} \tau_{1} \mathfrak{\Im} \tau_{2} \geq 1$. Applying it to

$$
\left[S_{1}, T^{-1}\right]=\left(\begin{array}{cc}
1-2 \tau_{2}+4 \tau_{2}^{2} & 4 \tau_{2} \\
2 \tau_{2}^{2} & 1+2 \tau_{2}
\end{array}\right)
$$

we see that $\Im \tau_{2} \geq 1 / 2$, and similarly applying it to $\left[T, S_{2}{ }^{-1}\right]$ we have $\Im \tau_{1} \geq 1 / 2$.

\section{Appendix 2}

To shed more light on the definition of canonical coordinates, we recall the familiar situation for $\Sigma_{1,1}$; see Birman and Series [4]. Consider first a Euclidean torus $\Sigma_{1,0}$ viewed as the quotient of $\mathbb{C}$ by translations $S: z \rightarrow z+1$ and $T: z \rightarrow z+i$. The unit

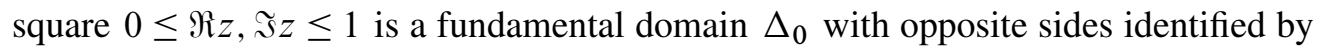
$S$ and $T$. Label each side by the translation which carries it to a paired side, so that the bottom side $\Im z=0$ is labelled $s_{T}$ since $T$ carries it to the top side $\Im z=1$; similarly the left side $\Re z=0$ is labelled $s_{S}$ since $S$ carries it to the right side $\Re z=1$. Any closed geodesic $\gamma$ on $\Sigma_{1,0}$ lifts to a line in $\mathbb{C}$. The lifts of $\gamma$ cut $\Delta_{0}$ in a number of 
pairwise disjoint parallel arcs running from one side of $\Delta_{0}$ to another: if the line has slope $q / p$ and we take $q \geq 0$, then there are $q$ strands which meet $s_{T}$ and $s_{T^{-1}}$, and $|p|$ strands which meet $s_{S}$ and $s_{S^{-1}}$. By collapsing all the strands which join a fixed pair of sides into one arc we obtain one of the four configurations shown in Figure 9, which we can view as four train tracks on $\Sigma_{1,0}$. Note that both the supporting train track and the weight on each branch is completely determined by the signed slope $q$ / $p$. For example, if $-1<q / p<0$, there is one branch from $s_{S}$ to $s_{S^{-1}}$ of weight $-p-q$ and there are branches joining $s_{S^{-1}}$ to $s_{T^{-1}}$ and $s_{T}$ to $s_{S}$ each of weight $q$, as shown in the lower left hand quadrant of Figure 9.

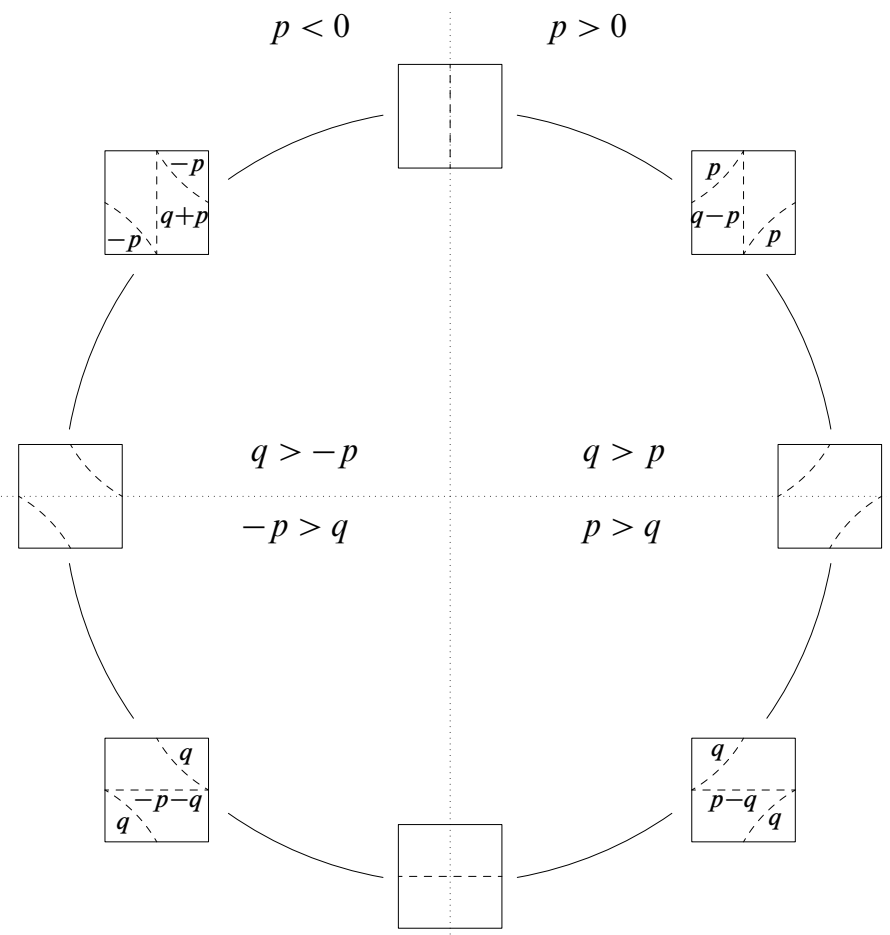

Figure 9: Canonical train tracks for the once punctured torus. In all charts, the total weight on the vertical sides is $|p|$ and on the horizontal sides is $q$.

We obtain the canonical coordinates for $\operatorname{ML}\left(\Sigma_{1,1}\right)$ by viewing $\Delta_{0}$ as a schematic representation of a fundamental domain for a hyperbolic once punctured torus $\Sigma_{1,1}$, with the puncture at the vertices. A simple closed curve on $\Sigma_{1,0}$ is also a simple closed curve on $\Sigma_{1,1}$, and every simple closed curve on $\Sigma_{1,1}$ can be represented in this way [4]. (The proof is to eliminate the possibility of nonzero weights on all four 
corner arcs and then use the switch conditions as in Section 4.) Thus any such curve is supported on one of the four train tracks in Figure 9 and the spaces of weights on these four tracks are a cell decomposition for $\operatorname{ML}\left(\Sigma_{1,1}\right)$ in the usual way.

Let $\gamma_{q / p} \in \mathcal{S}\left(\Sigma_{1,1}\right)$ be the curve associated to the line of slope $q / p$ in $\mathbb{C}$. The canonical coordinates $\mathbf{i}\left(\gamma_{q / p}\right)=(q, p) \in \mathbb{Z}_{+} \times \mathbb{Z}$ of $\gamma_{q / p}$ are the signed weights obtained as above from the original line in $\mathbb{C}$. We always take $q=i(S, \gamma) \geq 0$. We take $p>0$ if the line has positive slope, that is, if it contains an arc from $s_{S}$ to $s_{T^{-1}}$ and take $p<0$ if there is an arc from $s_{S^{-1}}$ to $s_{T^{-1}}$. (If $p=0$ the diagonal arcs have zero weight and we are on the boundary of two cells. The case $q=0$ corresponds to a single horizontal arc, for which we can take $p=1$ or $p=-1$.) It is easy to see from the above discussion that $\mathbf{i}\left(\gamma_{q / p}\right)$ determines both a train track on $\Sigma_{1,1}$ and the weights on that track, thus giving global coordinates for the homotopy classes of simple closed curves on $\operatorname{ML}\left(\Sigma_{1,1}\right)$. Note that $(q,|p|)$ are not in general equal to the weights on the branches of the track corresponding to $\gamma_{q / p}$. In fact, up to the choice of a base point for the twist, $p / q$ is the Dehn-Thurston twist coordinate of $\gamma$. These coordinates extend naturally by linearity and continuity to global coordinates for $\operatorname{ML}\left(\Sigma_{1,1}\right)$.

\section{Appendix 3}

We give the (presumably well known) formula for the bending angle $\phi$ between two consecutive segments of a geodesic $s$ on $\partial \mathcal{C}^{+}$which crosses a bending line $L$ of $\partial \mathcal{C}^{+}$ making an angle $\psi$ with $L$. Let the bending angle between the two planes $\Pi_{1}, \Pi_{2}$ which meet along $L$ be $\theta$. Let $s_{i} \subset \Pi_{i}$ be the two segments of $s$ which meet at $P \in L$. Measure $\phi$ so that $\phi=\theta$ when $\psi=\pi / 2$. The formula is

$$
\sin \phi / 2=\sin \psi \sin \theta / 2 .
$$

This can be proved by elementary Euclidean trigonometry. Let $N$ be the line perpendicular to $L$ which bisects the angle between $\Pi_{1}, \Pi_{2}$. The configuration of $L, \Pi_{1}, \Pi_{2}$ is invariant under rotation $\Omega$ by $\pi$ about $N$. Thus $\Omega$ interchanges $s_{1}$ and $s_{2}$ and $N$ is contained in the plane of $s_{1}$ and $s_{2}$.

Thinking of $N$ as "vertical", let $M$ be the "horizontal" line through $P$ perpendicular to $L$ and $N$. By definition of the bending angle and symmetry, $M$ makes an angle $\theta / 2$ with $\Pi_{2}$. Then the segment $s_{2}$ makes an angle $\phi / 2$ with the "horizontal" plane $H$ spanned by $L$ and $M$. Choose $X$ to be a point on $L$ at distance 1 from $P$. Assuming as we may that $\psi \neq \pi / 2$, let $Y \in \Pi_{2}$ be the point at which the perpendicular in $\Pi_{2}$ from $X$ meets $s_{2}$ and let $Z$ be the foot of the perpendicular from $Y$ to $H$. Then $\sin \phi / 2=|Z Y| /|P Y|=|Z Y| \cos \psi$ and $\sin \theta / 2=|Z Y| /|X Y|=|Z Y| / \tan \psi$. The result follows. 


\section{References}

[1] A Austin, Visualising the Maskit embedding of the twice punctured torus, MMath project, University of Warwick (2010)

[2] A Austin, C Series, Computing pleating rays for the twice punctured torus, in preparation

[3] A F Beardon, The geometry of discrete groups, Graduate Texts in Math. 91, Springer, New York (1983) MR698777

[4] J S Birman, C Series, Algebraic linearity for an automorphism of a surface group, J. Pure Appl. Algebra 52 (1988) 227-275 MR952081

[5] F Bonahon, J-P Otal, Laminations measurées de plissage des variétés hyperboliques de dimension 3, Ann. of Math. (2) 160 (2004) 1013-1055 MR2144972

[6] R D Canary, D B A Epstein, P Green, Notes on notes of Thurston, from: "Analytical and geometric aspects of hyperbolic space (Coventry/Durham, 1984)", (D B A Epstein, editor), London Math. Soc. Lecture Note Ser. 111, Cambridge Univ. Press (1987) 3-92 MR903850

[7] Y Chiang, Geometric intersection numbers on a five-punctured sphere, Ann. Acad. Sci. Fenn. Math. 26 (2001) 73-124 MR1816563

[8] Y-E Choi, K Rafi, C Series, Lines of minima and Teichmüller geodesics, Geom. Funct. Anal. 18 (2008) 698-754 MR2438996

[9] Y-E Choi, C Series, Lengths are coordinates for convex structures, J. Differential Geom. 73 (2006) 75-117 MR2217520

[10] D B A Epstein, A Marden, Convex hulls in hyperbolic space, a theorem of Sullivan, and measured pleated surfaces, from: "Analytical and geometric aspects of hyperbolic space (Coventry/Durham, 1984)”, (D B A Epstein, editor), London Math. Soc. Lecture Note Ser. 111, Cambridge Univ. Press (1987) 113-253 MR903852

[11] M Kapovich, Hyperbolic manifolds and discrete groups, Progress in Math. 183, Birkhäuser, Boston (2001) MR1792613

[12] L Keen, B Maskit, C Series, Geometric finiteness and uniqueness for Kleinian groups with circle packing limit sets, J. Reine Angew. Math. 436 (1993) 209-219 MR1207287

[13] L Keen, J R Parker, C Series, Pleating coordinates for the Maskit embedding of the Teichmüller space of punctured tori, Unpublished draft manuscript (1996)

[14] L Keen, J R Parker, C Series, Combinatorics of simple closed curves on the twice punctured torus, Israel J. Math. 112 (1999) 29-60 MR1715014

[15] L Keen, C Series, Pleating coordinates for the Maskit embedding of the Teichmüller space of punctured tori, Topology 32 (1993) 719-749 MR1241870

[16] L Keen, C Series, Pleating invariants for punctured torus groups, Topology 43 (2004) 447-491 MR2052972 
[17] I Kra, Horocyclic coordinates for Riemann surfaces and moduli spaces. I. Teichmüller and Riemann spaces of Kleinian groups, J. Amer. Math. Soc. 3 (1990) 499-578 MR1049503

[18] S Maloni, C Series, Top terms of trace polynomials in Kra's plumbing construction, Alg. Geom. Topol. 10 (2010) 1565-1607

[19] A Marden, Outer circles. An introduction to hyperbolic 3-manifolds, Cambridge Univ. Press (2007) MR2355387

[20] B Maskit, Kleinian groups, Grund. der Math. Wissenschaften 287, Springer, Berlin (1988) MR959135

[21] C McMullen, Cusps are dense, Ann. of Math. (2) 133 (1991) 217-247 MR1087348

[22] J Milnor, Singular points of complex hypersurfaces, Annals of Math. Studies 61, Princeton Univ. Press (1968) MR0239612

[23] Y N Minsky, Extremal length estimates and product regions in Teichmüller space, Duke Math. J. 83 (1996) 249-286 MR1390649

[24] H Miyachi, Cusps in complex boundaries of one-dimensional Teichmüller space, Conform. Geom. Dyn. 7 (2003) 103-151 MR2023050

[25] D Mumford, C Series, D Wright, Indra's pearls. The vision of Felix Klein, Cambridge Univ. Press (2002) MR1913879

[26] R C Penner, J L Harer, Combinatorics of train tracks, Annals of Math. Studies 125, Princeton Univ. Press (1992) MR1144770

[27] C Series, An extension of Wolpert's derivative formula, Pacific J. Math. 197 (2001) 223-239 MR1810217

[28] C Series, Limits of quasi-Fuchsian groups with small bending, Duke Math. J. 128 (2005) 285-329 MR2140265

[29] D Thurston, On geometric intersection of curves in surfaces Available at http:// www . math . columbia. edu/ dpt/

[30] D J Wright, The shape of the boundary of Maskit's embedding of the Teichmüller space of once punctured tori, Unpublished preprint (1988)

Mathematics Institute, University of Warwick

Coventry CV4 7AL, UK

C.M.Series@warwick.ac.uk

http: //www. warwick.ac.uk/ masbb

Proposed: Jean-Pierre Otal

Seconded: Colin Rourke, Joan Birman
Received: 6 January 2009

Revised: 30 June 2010 